\begin{abstract}
The fourth age of quantum chemistry offers fully flexible, black-box-type protocols for the accurate and detailed study of nuclear motions, applicable equally well to semirigid, floppy, flexible, polytopic, fluxional, and quasistructural polyatomic molecular systems, including complexes and clusters. Several codes, based on advanced fourth-age protocols, have been developed for the variational (or variational-like) solution of the timeindependent nuclear-motion (rotational-vibrational) Schrödinger equation. These codes yield accurate rovibrational energy levels, wavefunctions, and to some extent quantumnumber assignments for bound, resonance, and scattering states, revealing important spectroscopic and dynamical characteristics about the systems studied. When no approximations are introduced to the kinetic energy part of the rovibrational Hamiltonian, the accuracy of the computed results, assuming the validity of the Born-Oppenheimer approximation, depends solely on the accuracy of the representation of the potential energy surface utilized during these computations. From the point of view of potential applications it is important to emphasize that the most general codes can be employed both in full and any number of reduced dimensions. Several a posteriori analysis tools are available to improve the understanding of the extreme amount of numerical results produced by the stationary-state nuclear-motion computations. As shown through a few examples, these stationary-state solutions can straightforwardly be utilized for detailed quantum-dynamics studies. The applications briefly detailed at the end of this chapter help appreciate the power of the fourth-age quantum-chemical techniques developed and available to the spectroscopic and dynamics communities.
\end{abstract}

Keywords: bound and unbound rovibronic states; nuclear motion theory; flexible, polytopic, fluxional, and quasistructural molecules; quantum dynamics 


\title{
Exact numerical methods for stationary-state-based quantum dynamics of complex polyatomic molecules
}

\author{
Attila G. Császár, Csaba Fábri, and Tamás Szidarovszky
}

April 22, 2019

\section{Contents}

1 Introduction 4

2 Molecular Hamiltonians $\quad 8$

2.1 Coordinate systems . . . . . . . . . . . . . . . . 9

2.2 Formulation of the classical Hamiltonian in generalized internal coordinates . . 11

2.3 Formulation of the quantum-mechanical Hamiltonian in generalized internal coordinates . . . . . . . . . . . . . . . 13

2.4 Body-fixed frame embeddings . . . . . . . . . . . . . . . 14

2.5 Potential energy hypersurfaces $\ldots \ldots \ldots \ldots$

2.6 Basis sets and representations . . . . . . . . . . . . . 16

2.7 Determination of eigenstates $\ldots \ldots \ldots \ldots \ldots$

3 Computation of bound rovibrational states 19

3.1 On the variational solution $\ldots \ldots \ldots \ldots$

3.2 Symmetry in nuclear-motion computations . . . . . . . . . . . . . . . 22

3.3 Nuclear spin statistics . . . . . . . . . . . . . . . . . . . . . . 24

3.4 Wavefunction analysis tools via projection techniques . . . . . . . . . . . 25

4 Computation of rovibrational resonances 26

4.1 The stabilization method . . . . . . . . . . . . . . 27 
4.2 The technique of complex coordinate scaling (CCS) . . . . . . . . . 28

4.3 Complex absorbing potentials $(\mathrm{CAP}) \ldots \ldots \ldots \ldots \ldots$

4.4 Wavefunction analysis tools . . . . . . . . . . . . . . . . . . 29

5 Applications 29

5.1 Computation of all the bound (ro)vibrational eigenstates $\ldots \ldots$. . . . . . 30

$5.1 .1 \mathrm{H}_{2}{ }^{16} \mathrm{O}$ and its isotopologues $\ldots \ldots \ldots \ldots$

$5.1 .2 \mathrm{H}_{3}^{+}$and its deuterated isotopologues $\ldots \ldots \ldots . \ldots . \ldots 31$

5.2 Rovibrational computations on quasistructural molecules . . . . . . . . . 32

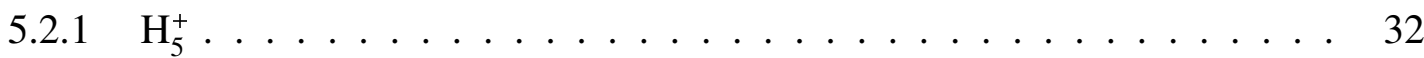

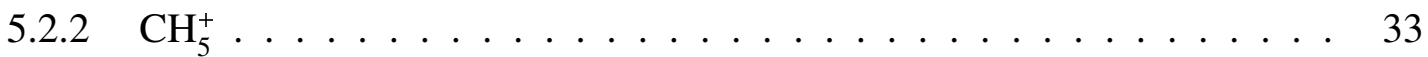

5.3 Computation of rovibrational resonances . . . . . . . . . . . . 34

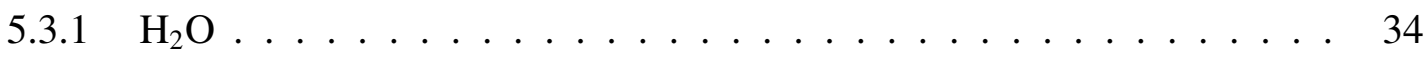

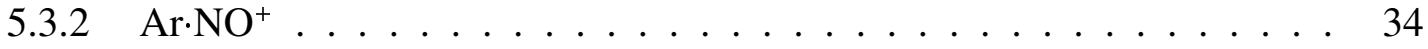

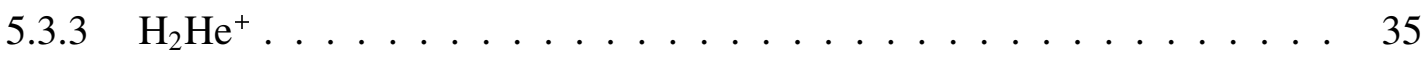

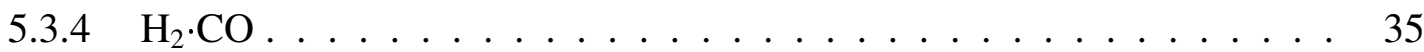

5.4 Stationary-state computations serving dynamical studies $\ldots \ldots$. . . . . 35

6 Summary and outlook 36 


\section{Introduction}

Over the last few decades there has been a steady increase in the number of gas-phase species, including molecules, molecular complexes, and clusters, in all feasible neutral and charged (cationic and anionic) forms, whose internal motions (rotations and vibrations) have been studied at high resolution by advanced techniques of molecular spectroscopy (Merkt and Quack, 2011). For systems confined to a finite region of configuration space or adsorbed on a surface, translational degrees of freedom (dof) may also need to be considered explicitly in a quantum treatment of nuclear motions (Dosch, 2001, Xu et al., 2008a, 2009b, 2008b, 2009a, McAfee and Poirier, 2009, Matanović et al., 2009, McAfee and Poirier, 2011, Matanović et al., 2012, Xu et al., 2013, Firmino et al., 2014, Xu et al., 2015, Poirier, 2015, Felker and Bačić, 2017, Zanuttini et al., 2018); nevertheless, such systems and situations are not considered in what follows.

It is highly desirable, almost mandatory in certain cases, to accompany high-resolution experimental spectroscopic studies with ever more sophisticated quantum-chemical [electronic structure (Helgaker et al., 2000, Császár et al., 2000) and nuclear motion (Merkt and Quack, 2011, Bowman et al., 2008, Császár et al., 2012, Tennyson, 2016, Carrington, 2017)] computations (note that the emphasis here is on the joint sophisticated treatment of the two distinct but equally relevant fields of quantum chemistry for a proper dynamical description of simple as well as complex molecular systems). This is especially important when the experimental absorption, emission, and action spectra become highly complex, in the simplest sense dense, which happens due to (a) the sheer size of the molecule under investigation, (b) the complexity of the internal motions when the dynamics leaves the so-called "semirigid" region [in cases when the results based on the rigid rotor (RR) (Kroto, 1992) and harmonic oscillator (HO) (Wilson Jr. et al., 1955) approximations, perhaps after a slight extension based on second-order vibrational perturbation theory (VPT2) (Papoušek and Aliev, 1982, Nielsen, 1951, Clabo Jr. et al., 1988, Allen et al., 1990, Barone et al., 2014), provide an outstanding qualitative and even a semiquantitative understanding of spectral regularities, the molecule is said to be semirigid], (c) high level of rovibronic excitation (at higher excitation energies all molecules become "floppy"), and (d) interaction of several electronic states [in what follows we mostly neglect these cases, focusing our attention on a single, well-separated electronic state, by default the ground electronic state (Császár et al., 2000)]. In the 21st century sophisticated quantum-chemical computations have been used to aid (or even make possible) the deduction of information encoded in exceedingly complex observed spectra.

There is growing evidence that the rovibrational energy-level structure of certain molecular species, due to their complex rovibrational dynamics, defies the conceptually simple, traditional interpretation attempts, building upon the RRHO approximation. This means that certain molecular parameters [e.g., the geometric structure (Demaison et al., 2011) and the rotational constants (Kroto, 1992) corresponding to a minimum on the potential energy surface (PES)] as well as the rovibrational energy level structure of these species strongly disagree with the corresponding data computed by (even the most sophisticated) electronic-structure techniques (Helgaker et al., 2000) within the RRHO approximation. It is thus important to note that the structure and spectra of "flexible", "polytopic" (Clementi et al., 1973, Nielsen et al., 1997), "fluxional" (McKee, 2011), and "quasistructural" (Fábri et al., 2014c, 2017, Fábri and 
Császár, 2018, Császár et al., 2019) molecules, the course of reactions and collisions of molecular systems (Schinke, 1993, Wyatt and Zhang, 1996, Zhang, 1999), and the extended dynamics characterizing rovibrational resonances (Papp et al., 2017a,b, 2018), playing a special role, for example, in bimolecular reactions, can all be computed and interpreted via sophisticated fourthage (Császár et al., 2012) variational quantum-chemical techniques. In this chapter all timeindependent nuclear-motion techniques which employ basis sets, represent the rovibrational Hamiltonian, $\hat{H}_{\mathrm{VR}}$, in this basis as a matrix, $\mathbf{H}$, and solve the resulting matrix eigenvalue problem via a "diagonalization" technique will be referred to as "variational", irrespective whether the matrix elements are computed accurately or not (Szalay et al., 2012). Detailed description of the best of these variational techniques utilizing exact kinetic energy operators expressed in curvilinear internal (shape) coordinates is the main aim of the first part of this chapter. This restriction on the algorithms addressed is dictated partly by the fact that only these techniques will generally be applicable for complex stationary-state-based dynamical computations. This choice also means that certain popular nuclear-motion techniques, especially those built upon the Eckart-Watson Hamiltonians (Eckart, 1935, Watson, 1968, 1970), are not considered here in detail. The reader is referred to review articles (Bowman et al., 2008, Carrington, 2017, Carney et al., 1978, Beck et al., 2000, Christiansen, 2007, Neff and Rauhut, 2009, Seidler et al., 2010) if interested in other extremely useful nuclear-motion techniques, whose applicability, however, is basically constrained to low excitations of semirigid molecular systems. It is also not the purpose of this chapter to discuss certain highly useful simplification techniques (Sadri et al., 2014) built upon certain choices of the form of the PES or computational techniques (Fábri et al., 2014a, Pavlyuchko et al., 2015) not designed to treat systems exhibiting arbitrarily large-amplitude motions (LAM).

At this point it is instructive to return briefly to what is considered to be the standard model in the field of stationary rovibrational states of free molecules. First, without external forces the three translational dofs of molecular systems can be separated exactly from the rest of the motions. Second, the approximate separation of the three rotational dofs from the $3 N-6$ vibrational dofs of an $\mathrm{N}$-atomic nonlinear molecule is part of the RRHO-based treatment of molecular vibrations and rotations (Wilson Jr. et al., 1955, Kroto, 1992). Third, using normal coordinates within the HO approximation to the PES leads to perfect separation of the vibrational modes, i.e., to onedimensional (1D) model vibrations [the adiabatic separation of certain molecular vibrations remains a highly useful concept much beyond the HO approximation (Hougen et al., 1970, Miller et al., 1980, Carrington Jr. and Miller, 1984, Lauvergnat, 2001, Fehrensen et al., 2003)]. Finally, note that in linear molecules, including diatomics, rotation around the internuclear axis is not a proper internal motion. Therefore, these molecules have only two rotational dofs and one extra vibrational dof.

As the next point, it is noted that observable rovibrational energy-level splittings, assuming a molecular Hamiltonian without hyperfine interaction terms, are considered by many to be due to (multidimensional) quantum tunneling (Hund, 1927, Nordheim, 1928, Frenkel, 1932, Bell, 1980) (think of ammonia and its "umbrella" motion (Al Derzi et al., 2015) as a principally 1D tunneling case). Clearly, if tunneling occurs in a molecule then all of its vibrational and rotational dofs are affected (Császár and Furtenbacher, 2016). This simple picture is complicated by the fact that splitting, perhaps with a rather complex splitting pattern (Fábri et al., 2017, Fábri and Császár, 2018), also occurs if LAMs among symmetrically equivalent versions (LonguetHiggins, 1963, Bunker and Jensen, 1998) of a molecule take place. A LAM coordinate can 
be associated with chemical isomerization [e.g., $\mathrm{HNC} \rightarrow \mathrm{HCN}$ for the $[\mathrm{H}, \mathrm{C}, \mathrm{N}]$ system (van Mourik et al., 2001, Mellau, 2011a,b)], but here the picture of tunneling splitting is less useful due partly to the asymmetry of the wells. Energy-level splitting in just slightly asymmetric potentials introduces interesting dynamical phenomena, like tunneling switching (Quack, 1986, Albert et al., 2013, 2016, Šmydke et al., 2019). Deep tunneling (Bell, 1980, Schreiner et al., 2008) along a single path can be treated as a simple, well understood and utilized concept similar in spirit to the RRHO approximation. However, there are complex motions where treatment of tunneling and energy-level splittings requires sophisticated variational modeling efforts, way beyond the simple 1D picture. It must be emphasized that often it is hard to separate tunneling and other large-amplitude motions. For the variational nuclear-motion techniques described in this chapter this separation causes no particular difficulties.

A molecule is considered semirigid if its (ground) electronic state contains a single, welldefined, and conveniently deep minimum. For the $3 N-3$ internal degrees of freedom of a semirigid molecule the rovibrational eigenstates can be labelled by the irreducible representations (irreps) of the point group [isomorphic to a molecular symmetry (MS) group (Longuet-Higgins, 1963, Bunker and Jensen, 1998) for semirigid molecules] characterizing the unique equilibrium structure of the molecule, the vibrational spacing decreases, almost without exception, with the increase in vibrational excitation, and the rotational states can be assigned to a certain vibrational state, as implied by the RRHO approximation (from now on these vibrational states will be called the vibrational parents (Mátyus et al., 2010, Wang et al., 2011, Szidarovszky et al., 2012) of certain rovibrational states). For molecules considered to be semirigid, (a) the vibrational and rotational motions can be treated separately (they have sufficiently different energy and time scales); (b) the rotational spectrum provides important and accurate information about the (temperature-dependent, effective) structure (Demaison et al., 2011) of the molecule; and (c) the simple RR picture is perfectly adequate to explain not only the characteristics of the observed microwave (MW) or millimeterwave (MMW) spectra, though hyperfine splittings may also need to be considered, but it is also sufficient to derive the (equilibrium) structure (Demaison et al., 2011) of the molecule via the determination of rotational constants [the lowest-order terms in an effective rotational Hamiltonian (Kroto, 1992)]. For semirigid molecules there are traditional quantum-chemical treatments, such as vibrational perturbation theory based on the Eckart-Watson (EW) Hamiltonians (Eckart, 1935, Watson, 1968, 1970) carried out to second order (Nielsen, 1951, Mills, 1972, Papoušek and Aliev, 1982) and beyond (Sibert III, 1988, Aarset et al., 2000, Iung et al., 2006) or even variational treatments utilizing the exact EW Hamiltonians. Note that extensions offered to the variational EW Hamiltonian treatment [like the Reaction Path Hamiltonian (RPH) (Miller et al., 1980) extension of the widely utilized protocol and code Multimode (Carter et al., 1998, Bowman et al., 2003)] would still allow the treatment of a LAM only along a single coordinate.

As noted above, there is a large number of molecules for which RRHO-based treatments are not adequate. The extent how far one needs to go beyond the RRHO, the VPT2, and the EW-based variational treatments depends greatly upon the complexity of the nuclear motions. Nuclear motions of molecules exhibiting LAM, as well as polytopic, fluxional, and quasistructural molecules require quantum treatments of differing sophistication. Furthermore, treating dynamical processes (e.g., reactions and collisions) also calls for sophisticated variational procedures. Dealing with motions of such molecules and molecular systems leads us closer to the techniques required in complex quantum-dynamical treatments. Systems where understanding 
complex internal motions, as well as their spectral manifestations, required the development of fourth-age variational quantum-chemical techniques insensitive to the number of minima and the corresponding equilibrium structures, $r_{\mathrm{e}}^{\mathrm{BO}}$, the PES possesses are as follows: fluxional (McKee, 2011), polytopic (Clementi et al., 1973, Nielsen et al., 1997, Bogey et al., 1991), and quasistructural (Fábri et al., 2014c, 2017, Fábri and Császár, 2018, Mills and Thompson, 1954, Bunker and Longuet-Higgins, 1964, Sarka et al., 2015, Sarka and Császár, 2016, Császár et al., 2019) molecules, as well as van der Waals (vdW) complexes (Nesbitt and Naaman, 1989, Sarka et al., 2016, 2017).

In the fourth age of quantum chemistry (Császár et al., 2012) the nuclear motions of rigid, semirigid, floppy, flexible, polytopic, fluxional, and quasistructural molecular systems, including molecular clusters, van der Waals complexes, and molecules exhibiting highly unusual bonds, like three-center-two-electron (3c-2e) bonds [e.g., in protonated $\mathrm{H}_{2}, \mathrm{H}_{3}^{+}$(Polyansky et al., 2012), and protonated methane, $\mathrm{CH}_{5}^{+}$(Fábri et al., 2017)], can all be treated with sophisticated variational techniques solving the time-independent nuclear Schrödinger equation in a numerically exact fashion. Time-independent variational techniques employed in computational molecular spectroscopy, a field of nuclear-motion theory, have traditionally been built around the use of rectilinear normal coordinates, the Eckart embedding, and the resulting EW Hamiltonians (Eckart, 1935, Watson, 1968, 1970). Nevertheless, for large-amplitude motions one must use curvilinear internal coordinates as only they provide a suitable and efficient, physically motivated description of the dynamics and allow to move away arbitrarily far from a single reference configuration and allow the use of arbitrary body-fixed frame embeddings. Furthermore, since in many cases there is only a small number of (but more than one) internal coordinates that dominate the large-amplitude motions (the other vibrational dofs can be considered as "spectator" modes) even in relatively complex and large molecules, development of reduceddimensional (variational) nuclear-motion treatments is highly desirable. It is hard to expect that "regular" stretching dofs will have a significant role in complex nuclear dynamics, unless dissociation is part of the motions considered. On the other end of vibrational softness, torsional vibrations and other LAMs appear to be the best candidates of the vibrational dofs which could couple extremely strongly with the rotational dofs. During the last two decades efficient and black-box-type protocols relying on the use of a Hamiltonian expressed in arbitrary internal coordinates and body-fixed frames have been developed (Fehrensen et al., 1999a,b, Luckhaus, 2000, 2003, Lauvergnat and Nauts, 2002, Yurchenko et al., 2007, Mátyus et al., 2009, Changala, 2019). The most desirable features of these protocols are as follows: (a) they are completely general in the sense that a single code can treat all molecular systems of feasible size, irrespective of the fact whether their PESs contain a single minimum or easily accessible multiple minima and what the choices of internal coordinates and coordinate system embeddings are; (b) the always very complex form of the exact kinetic energy operator in internal coordinates, see, e.g., Handy (1987), Császár and Handy (1995a), and Császár and Handy (1995b), may not need to be known explicitly; and (c) they allow the use of arbitrary reduced-dimensional nuclear motion treatments and PESs of arbitrary functional form within the same code. All these features of a time-independent variational nuclear-motion protocol become especially important when the aim is to treat large-amplitude motions of larger fluxional and quasistructural molecular systems or highly excited rovibrational states of molecules [the extreme case is the determination of the complete set of rovibrational (bound) states (Szidarovszky et al., 2010, Császár et al., 2010)]. Furthermore, these are the computations which can provide all the necessary rovibrational states for dynamical studies. Several codes have been developed which can perform these numerically 
very demanding quantum-chemical computations: ElVibRot (Lauvergnat and Nauts, 2002, Lauvergnat, 2019), GENIUSH (Mátyus et al., 2009, Fábri et al., 2011a), MCTDH (Meyer et al., 2009), NITROGEN (Changala, 2019), TROVE (Yurchenko et al., 2007), codes due to Carrington (Manzhos et al., 2009, 2015, Manzhos and Carrington, Jr., 2016), Poirier (Poirier, 2003b, Chen and Poirier, 2006, 2010, Petty and Poirier, 2014), Yu (Yu, 2002, 2004a,b, 2006), and perhaps there are others, as well. Note that for triatomic systems, for which a completely general internal-coordinate Hamiltonian, often referred to as the Sutcliffe-Tennyson Hamiltonian (Sutcliffe and Tennyson, 1991), could be developed, there are lot more codes available which are similar in spirit to those mentioned above, e.g., DVR3D (Tennyson et al., 2004), ScalIT (Petty and Poirier, 2014), D²FOPI (Szidarovszky et al., 2010), and codes due to Guo (Ma et al., 1999) and Schwenke (Schwenke, 1992, Klepeis et al., 1993). For four-atomic molecules it is not possible to develop a Hamiltonian which would cover all possible internal coordinate systems. Nevertheless, there are excellent variational nuclear-motion codes which can handle a number of internal coordinate systems, e.g., WAVR4 (Kozin et al., 2004, 2005) and codes developed by Schwenke (Schwenke, 1996) and Mladenović (Mladenović, 2002a,b).

It is appropriate to add at the end that there are significant efforts and advanced variational codes which do not make the BO separation of nuclear and electronic dofs and allow the "full" treatment of quantum systems, at present up to five bodies (Armour et al., 2005, Stanke et al., 2006, Pavanello et al., 2010, Pachucki and Komasa, 2010, Mátyus et al., 2011, Mátyus and Reiher, 2012, Mátyus, 2013). These extremely accurate computations are not yet applicable for polyelectronic and polyatomic systems and the eigenstates may not be amenable to a straightforward dynamical interpretation.

\section{Molecular Hamiltonians}

Let our isolated molecular system contain $N$ nuclei with masses $m_{i}, i=1, \ldots, N$, and let $\mathbf{X}_{i}$ be the position vectors of the nuclei in the space-fixed (SF) Cartesian coordinate frame $(X, Y, Z)$. The set of nuclear positions $\left\{\mathbf{X}_{1}, \mathbf{X}_{2}, \ldots, \mathbf{X}_{N}\right\}$ is called the configuration of the molecular system. Then,

$$
\mathbf{X}_{i}=\mathbf{X}^{\mathrm{COM}}+\mathbf{R} \mathbf{x}_{i}, \quad i=1, \ldots, N,
$$

where $\mathbf{R}$ is an orthogonal rotation matrix depending on the three rotational coordinates $\rho_{1}$, $\rho_{2}$, and $\rho_{3}$, and $\mathbf{x}_{i}=\mathbf{x}_{i}(t)$ are the body-fixed position vectors of each nucleus, a function of time $t$, in the body-fixed (BF) reference frame $(x, y, z)$, and COM stands for center-of-mass. It is customary to describe the motions of the molecular system in terms of some set of scalar variables $q_{i}(t)\left(i=1,2, \ldots, A_{\mathrm{s}}\right)$. The $\mathbf{x}_{i}=\mathbf{x}_{i}\left(q_{1}, q_{2}, \ldots, q_{A_{s}} ; t\right) \mathrm{BF}$ atomic position vectors are functions of the $q_{i}$ internal (shape) coordinates, of which $A_{\mathrm{s}}$ are active $\left(A_{\mathrm{s}}<3 N-6\right.$ holds for reduced-dimensional, while $A_{\mathrm{s}}=3 N-6$ holds for full-dimensional rovibrational treatment of nonlinear molecules). Furthermore, let $V$ denote the PES of the system, depending on a given set of shape coordinates (in this chapter we are dealing solely with conservative forces). 


\subsection{Coordinate systems}

When choosing the $3 N$ coordinates, sufficient to describe the complete nuclear dynamics of an $\mathrm{N}$-atomic molecular system, it is desirable to choose coordinates which simultaneously fulfill the criteria of simplicity, separability, and factorizability. Since there is no single coordinate system which exhibits all these important characteristics at the same time, several proposals have been made (Stefanski and Taylor, 1985, Bačić and Light, 1989, Colbert and Sibert III, 1989, Bramley et al., 1991, Mayrhofer and Sibert III, 1995, Rauhut, 2007, Yagi et al., 2012, Thomsen et al., 2014, Klinting et al., 2015) how to generate optimal/optimized coordinate systems for treating nuclear dynamics.

Simplicity in this context means that the form of the kinetic energy operator (KEO), based on the chosen set of coordinates, is as simple as possible. The simplest way to achieve simplicity goes through the selection of orthogonal shape coordinates, including the Jacobi (body-fixed scattering) (Jacobi, 1843, Smith, 1959, Tennyson and Sutcliffe, 1982) and Radau (Radau, 1868, Smith, 1980) coordinates.

Separability means that the complete nuclear-motion (rotational-vibrational) Hamiltonian, $\hat{H}_{\mathrm{VR}}$, can be written as the finite sum of independent operators:

$$
\hat{H}_{\mathrm{VR}}=\hat{H}_{1, i}\left(q_{1}, \ldots, q_{i}\right)+\hat{H}_{i+1, j}\left(q_{i+1}, \ldots, q_{j}\right)+\ldots+\hat{H}_{k, A_{\mathrm{s}}}\left(q_{k}, \ldots, q_{A_{\mathrm{s}}}\right)+\hat{H}^{\prime}\left(q_{1}, \ldots, q_{A_{\mathrm{s}}}\right),
$$

and $\hat{H}^{\prime}$ remains small. In an ideal case each term is one-dimensional and the product(s) of the one-dimensional eigenfunctions approximates well the exact eigenfunction(s) of $\hat{H}_{\mathrm{VR}}$. We talk about exact separation when $\hat{H}^{\prime}=0$, but this is rarely the case. Note that in a field-free case the translational motion can be separated exactly from the other nuclear motions.

Factorizability means that the complete Hamiltonian can be written as the sum of products (SOP) (Carrington, 2017) of one-dimensional operators,

$$
\hat{H}_{\mathrm{VR}}=\sum_{i} \prod_{j=1}^{A_{\mathrm{s}}+3} \hat{H}_{i j}\left(q_{j}\right) .
$$

This form of the Hamiltonian is advantageous from a computational point of view, as well, as the matrix elements of a representation of $\hat{H}_{\mathrm{VR}}$ of this form can be computed through simple one-dimensional integrations.

Next, let us briefly discuss the different choices of shape coordinates that can be employed during nuclear-motion computations.

Let us start with a set of rectilinear Cartesian coordinates. Although Cartesian coordinates are simple and their use is simple, as well, as in Cartesian coordinates both the classical and the quantum-mechanical expressions for the kinetic energy have by far the simplest form, Cartesian coordinates are considered to be not well suited for rovibrational computations. Nevertheless, some aspects of the use of SF Cartesians in variational nuclear motion computations have been explored (Blanco and Heller, 1983, Broeckhove and Lathouwers, 1993, Suarez et al., 2009, Manzhos and Carrington, Jr., 2016). Manzhos and Carrington, Jr. (2016) demonstrated that if the kinetic energy operator is expressed in space-fixed Cartesians but the basis functions depend only on shape coordinates then one obtains only vibrational energy levels. The use 
of KEOs in SF Cartesians is still uncommon, partly because these days derivation (Handy, 1987, Lukka, 1995, Császár and Handy, 1995a,b, Pesonen, 2013, 2014) or numerical handling (Meyer, 1979, Laane et al., 1982, Harthcock and Laane, 1982, McCoy et al., 1991, Luckhaus, 2000, Lauvergnat and Nauts, 2002, Mátyus et al., 2009, Fábri et al., 2011a) of kinetic energy operators in curvilinear coordinates is relatively straightforward. In nuclear-motion theory there are only a few methods, like the quantum Monte Carlo technique (Hammond et al., 1994), which work in Cartesian space, mostly out of algorithmic convenience.

Normal coordinates are defined in quantum chemistry analogously to their classical mechanical definition (Hestenes, 1999, Goldstein et al., 2014). They are defined with respect to a single minimum and they are excellent choice for the description of small-amplitude vibrations of semirigid molecules. Nevertheless, normal coordinates are unsuitable to describe LAMs as they are strongly attached to a single deep minimum and its immediate surrounding. Note that not only rectilinear but also curvilinear normal coordinates have been defined (Quade, 1976).

Curvilinear internal (shape) coordinates appear to be the best choice for performing variational nuclear-motion computations. Even in harmonic vibrational analysis (Wilson Jr. et al., 1955, Califano, 1970) they have been employed extensively by spectroscopists and quantum chemists (Pulay et al., 1979). Properly selected internal coordinates can describe straightforwardly the full configuration space. For variational nuclear-motion computations the use of orthogonal variants (e.g., generalized Jacobi (Jacobi, 1843, Hirschfelder and Dahler, 1956, Jepsen and Hirschfelder, 1959, Smith, 1959, Pack, 1984) and Radau (Radau, 1868, Smith, 1980) coordinates) is advantageous, so they should be selected whenever feasible. For traditional reasons, bond length-bond angle coordinates are often referred to as valence coordinates. A number of KEOs have been obtained analytically for valence coordinates (Handy, 1987, Sutcliffe and Tennyson, 1991, Chapuisat and Iung, 1992, Császár and Handy, 1995a,b, Lukka, 1995, Mladenović, 2000b, Pesonen and Halonen, 2003). Curvilinear internal coordinates tend to reduce the coupling in the potential, though usually at the expense of enhanced coupling in the KEO. Polyspherical coordinates (Chapuisat and Iung, 1992, Gatti and Iung, 2009, Mladenović, 2000a,b,c), providing a spherical polar parametrization, have also been used to derive analytic KEOs for nuclear-motion computations. For an $N$-atomic molecule polyspherical coordinates are comprised of $N-1$ radial coordinates ("stretches") and $2 N-5$ angular coordinates, usually further classified to $N-2$ bends and $N-3$ torsional (dihedral) angles in a spherical polar parametrization. Many valence coordinate systems can be considered as polyspherical coordinate systems. As to hyperspherical coordinates, they are defined by one hyperradius, and all the other coordinate elements are hyperangles (Delves, 1959, Smith, 1959, Louck and Galbraith, 1972, Johnson, 1980, 1983, Pack, 1984). Hyperspherical coordinates have special symmetry properties, these can be exploited during nuclear-motion computations (Louck and Galbraith, 1972).

A difficulty in nuclear-motion computations of flexible systems is that if one uses normal coordinates or certain internal coordinates not describing well the actual complex motions of the system, the basis functions associated with these coordinates are often strongly coupled. A judicious choice of internal coordinates and basis functions is thus very basic to converge the usually very large number of computed (ro)vibrational eigenstates (De Leon and Heller, 1984, Colbert and Sibert III, 1989) and allow for the improved interpretation of the wavefunctions, important to understand the dynamics of the system.

From what is noted above it follows that the choice of curvilinear (generalized, orthogonal) 
internal coordinate systems is by far the best choice for variational nuclear-motion computations (Watson, 2004). Redundancy conditions (Wilson Jr. et al., 1955, Califano, 1970, Hoy et al., 1972) should always be checked when selecting the shape coordinates.

\subsection{Formulation of the classical Hamiltonian in generalized internal co- ordinates}

We follow the generally applied approach to derive the quantum Hamiltonian by first deriving the classical Lagrangian and Hamiltonian forms and then performing the quantization. The nonrelativistic Lagrangian $L$ of the molecule is of the form (Sørensen, 1979, Hestenes, 1999, Pesonen, 2013, 2014)

$$
L=\frac{1}{2} \sum_{k=1}^{A_{\mathrm{s}}+6} \sum_{l=1}^{A_{\mathrm{s}}+6} g_{q_{k} q_{l}} \dot{q}_{k} \dot{q}_{l}-V
$$

where

$$
g_{k l} \equiv g_{q_{k} q_{l}}=\sum_{i=1}^{N} m_{i} \frac{\partial \mathbf{X}_{i}^{\mathrm{T}}}{\partial q_{k}} \frac{\partial \mathbf{X}_{i}}{\partial q_{l}}=\sum_{i=1}^{N} m_{i} \mathbf{t}_{i k}^{\mathrm{T}} \mathbf{t}_{i l}, \quad k, l=1, \ldots, A_{\mathrm{s}}+6
$$

is the covariant metric tensor of the coordinate transformation from the space-fixed massweighted Cartesian coordinates to the generalized coordinates $q_{k}$, based on the covariant measuring vectors $e_{k}^{(i)}=\frac{\partial \mathbf{X}_{i}}{\partial q_{k}}$ (Hestenes, 1999). As in Eq. (5), the covariant measuring vectors are often referred to as the $\mathbf{t}$ vectors (Sørensen, 1979), corresponding to the $q_{k}$ generalized coordinate on atom $i$.

The classical Hamiltonian can then be expressed as

$$
H=\frac{1}{2} \sum_{k=1}^{A_{\mathrm{s}}+6} \sum_{l=1}^{A_{\mathrm{s}}+6} G^{k l} p_{k} p_{l}+V
$$

where $\mathbf{G}=\mathbf{g}^{-1}$ is the contravariant metric tensor and $p_{k}=\frac{\partial L}{\partial \dot{q}_{k}}\left(k=1, \ldots, A_{\mathrm{s}}+6\right)$ is the canonical momentum conjugate to $q_{k}$. There is a well-known reciprocality relation (Hestenes, 1999) connecting the covariant and contravariant measuring vectors:

$$
\sum_{i=1}^{N} e_{j}^{(i)} \cdot e_{(i)}^{k}=\delta_{j k}
$$

The contravariant measuring vectors are known in the theory of the rovibrational motion of molecules as s vectors (Wilson Jr. et al., 1955, Pesonen, 2014).

To construct the covariant $\mathbf{g}$ and the contravariant $\mathbf{G}$ metric tensors, let us describe the configuration of the system by the $q_{k}=q_{k}$ active $\left(k=1, \ldots, A_{\mathrm{s}}\right)$ and constrained $\left(k=A_{\mathrm{s}}+\right.$ $1, \ldots, 3 N-6)$ internal coordinates, the three rotational $\left(\rho_{1}, \rho_{2}\right.$, and $\left.\rho_{3}\right)$ and the three center-ofmass $\left(X_{1}^{\mathrm{COM}}, X_{2}^{\mathrm{COM}}\right.$, and $\left.X_{3}^{\mathrm{COM}}\right)$ coordinates describing the translation of the system. Derivation of the $g_{k l}$ matrix elements is equivalent, see Eq. (5), to constructing the $\mathbf{t}_{i k}$ vectors (Sørensen, 1979) in terms of the generalized coordinates. 
The translational $\mathbf{t}_{i, k+A_{\mathrm{s}}+3}(k=1,2,3)$ vectors are simply

$$
t_{i, k+A_{\mathrm{s}}+3}^{a}=\frac{\partial X_{i a}}{\partial X_{k}^{\mathrm{COM}}}=\delta_{a k}
$$

where $a=1,2,3$ refers to the three components of the vector $\mathbf{t}$. Thus, the translational $\mathbf{g}$ matrix elements are obtained as

$$
g_{k+A_{\mathrm{s}}+3, l+A_{\mathrm{s}}+3}=M \cdot \delta_{k l}, \quad k, l=1,2,3,
$$

where $M=\sum_{i=1}^{N} m_{i}$ is the total mass of the system.

The rotational-translational and the vibrational-translational coupling matrix elements of $\mathbf{g}$ are all equal to zero. Therefore, the COM motion can be separated exactly from the rest of the coordinates. This allows the introduction of the

$$
H^{\mathrm{int}}=T^{\mathrm{int}}+V=\frac{1}{2} \sum_{k=1}^{A_{\mathrm{s}}+3} \sum_{l=1}^{A_{\mathrm{s}}+3} G^{k l} p_{k} p_{l}+V
$$

internal-motion (rovibrational) Hamiltonian.

The rotational $\mathbf{t}_{i, k+A_{\mathrm{s}}}(k=1,2,3)$ vectors take the form

$$
t_{i, k+A_{\mathrm{s}}}^{a}=\frac{\partial X_{i a}}{\partial \rho_{k}}=\sum_{b=1}^{3} \frac{\partial R_{a b}}{\partial \rho_{k}} x_{i b}
$$

and the rotational $\mathbf{g}$ matrix elements are equal to

$$
g_{k+A_{\mathrm{s}}, l+A_{\mathrm{s}}}=\sum_{i=1}^{N} m_{i}\left(\mathbf{u}_{k} \times \mathbf{x}_{i}\right)^{\mathrm{T}}\left(\mathbf{u}_{l} \times \mathbf{x}_{i}\right),
$$

where the direction of the unit vector $\mathbf{u}_{k}$ coincides with the axis of rotation corresponding to the rotational coordinate $\rho_{k}$.

The vibrational $\mathbf{t}_{i k}\left(k=1, \ldots, A_{\mathrm{s}}\right)$ vectors are

$$
t_{i k}^{a}=\frac{\partial X_{i a}}{\partial q_{k}}=\sum_{b=1}^{3} R_{a b} \frac{\partial x_{i b}}{\partial q_{k}} .
$$

Thus, the vibrational $\mathbf{g}$ matrix elements are given as

$$
g_{k l}=\sum_{i=1}^{N} m_{i} \frac{\partial \mathbf{x}_{i}^{\mathrm{T}}}{\partial q_{k}} \frac{\partial \mathbf{x}_{i}}{\partial q_{l}}
$$

where $k, l=1, \ldots, A_{\mathrm{s}}$.

Finally, the rotational-vibrational coupling (often called Coriolis coupling) g matrix elements have the form

$$
g_{k, l+A_{\mathrm{s}}}=\sum_{i=1}^{N} m_{i} \frac{\partial \mathbf{x}_{i}^{\mathrm{T}}}{\partial q_{k}}\left(\mathbf{u}_{l} \times \mathbf{x}_{i}\right),
$$


where $k=1, \ldots, A_{\mathrm{s}}$ and $l=1,2,3$. To determine $\mathbf{g}$, the body-fixed frame embedding has to be defined, which gives the dependence of the $\mathbf{x}_{i}$ body-fixed nuclear position vectors on the $q_{k}$ shape coordinates (see also Section 2.4).

If larger molecules are examined, it is often necessary and at the same time a good approximation to introduce reduced-dimensional rovibrational models. The two possible ways of reducing the dimensionality of the problem are to delete rows and the corresponding columns of $\mathbf{g}$ or $\mathbf{G}$. The first case, when the $i$ th row and column $(i=1, \ldots, 3 N-6)$ of $\mathbf{g}$ are deleted, implies the $\dot{q}_{i}=0$ constraint, while the second approach, when the $i$ th row and column of $\mathbf{G}$ are discarded, is equivalent to $p_{i}=0$. If orthogonal coordinates are employed to describe the rovibrational dynamics of molecules, the two approaches are equivalent. However, in general the two strategies provide different reduced-dimensional models and numerical results (Fábri et al., 2009, Mátyus et al., 2009).

\subsection{Formulation of the quantum-mechanical Hamiltonian in generalized internal coordinates}

In this subsection the internal-motion (rovibrational) quantum-mechanical Hamiltonian $\hat{H}^{\text {int }}$ is introduced in analogy to the internal-motion (rovibrational) classical Hamiltonian $H^{\text {int }}$ (Nauts and Chapuisat, 1985, Littlejohn and Reinsch, 1997), see Eq. (10). Within the Born-Oppenheimer (BO) separation of nuclear and electronic dofs (Born and Oppenheimer, 1927), the potential energy acting on the nuclei, $\hat{V}$, can be obtained by electronic-structure computations (Murrell et al., 1984, Mezey, 1987, Császár et al., 2000, 2001) (see Section 2.5). Here, we focus on constructing the rovibrational kinetic energy operator in the set of $q_{k}\left(k=1, \ldots, A_{\mathrm{s}}\right)$ vibrational and $\rho_{k}(k=1,2,3)$ rotational coordinates. According to differential geometry (Podolsky, 1928, Schutz, 1980), $\hat{T}^{\text {int }}$ becomes

$$
\hat{T}^{\mathrm{int}}=\frac{1}{2} \sum_{k=1}^{A_{\mathrm{s}}+3} \sum_{l=1}^{A_{\mathrm{s}}+3} \tilde{g}^{-1 / 4} \hat{p}_{k}^{\dagger} G^{k l} \tilde{g}^{1 / 2} \hat{p}_{l} \tilde{g}^{-1 / 4},
$$

where $\tilde{g}=\operatorname{det}(\mathbf{g})$, the integration volume element contains no extra factors, and in units of $\hbar$, for the vibrational coordinates $\hat{p}_{k}=-\mathrm{i} \frac{\partial}{\partial q_{k}}\left(k=1, \ldots, A_{\mathrm{s}}\right)$, while for the rotational coordinates $\hat{p}_{k+A_{\mathrm{s}}}=-\mathrm{i} \frac{\partial}{\partial \rho_{k}}(k=1,2,3)$, where $\mathrm{i}^{2}=-1$.

Next, let us utilize that the angular momentum $\hat{\mathbf{J}}$ is the infinitesimal generator of rotations (Zare, 1988),

$$
\mathbf{n} \hat{\mathbf{J}}=-\mathrm{i} \frac{\partial}{\partial \phi},
$$

where $\mathbf{n}$ is a unit vector specifying the rotational axis and $\phi$ is an angle associated with the rotation. According to Eq. (17), it is straightforward to identify the rotational $\hat{p}_{k+A_{s}}$ operators as the projections of the total angular momentum. After specifying three unique rotational axes, three successive rotations can be performed, defining the transformation between the SF and BF frames. As the $\rho_{k}$ rotational coordinate describes a rotation around the $k$ th of the three rotational axes,

$$
\hat{p}_{k+A_{\mathrm{s}}}=-\mathrm{i} \frac{\partial}{\partial \rho_{k}}=\hat{J}_{k}
$$


where $\hat{J}_{k}$ is the component of the total angular momentum vector along the $k$ th rotational axis. If the three rotational axes are chosen to coincide with the three axes of the body-fixed frame, the $\hat{J}_{k}$ operators correspond to the angular momentum components expressed in the body-fixed frame, and the $\rho_{k}$ rotational angles define three successive rotations around the three orthogonal axes of the body-fixed system. It is advantageous to employ these infinitesimal rotational coordinates (Lukka, 1995, Colwell and Handy, 1997) instead of the widely-used Eulerian angles for at least two reasons: (a) one can directly insert the body-fixed components of the total angular momentum into the rovibrational Hamiltonian by utilizing Eq. (18); and (b) the matrix elements $g_{k l}$ can be computed trivially, as according to Eqs. (12) and (15) one only needs to evaluate the $\mathbf{u}_{k} \times \mathbf{x}_{i}$ cross products.

\subsection{Body-fixed frame embeddings}

While the SF form of the nuclear-motion Hamiltonian expressed in internal coordinates is available (Mladenović, 2000a), it is much more common to work with BF frame embeddings. Defining a convenient set of rotating $\mathrm{BF}$ axes has become an important issue in modern nuclearmotion theory (Born and Heisenberg, 1924, Louck and Galbraith, 1976, Bunker and Jensen, 1998, Mladenović, 2000a,b,c). The orientation of the BF axis system with respect to the SF frame is specified by the three rotational coordinates. Although all embeddings provide the same rovibrational energy levels and eigenstates during numerically exact variational rovibrational computations, it is of great importance to find embeddings that provide an optimal separation between rotations and vibrations. Many conceptually simple embeddings based on geometric definitions do not meet this requirement. For example, in the case of $\mathrm{XY}_{2}$ molecules the bisector embedding, especially if it is coupled with the choice of orthogonal Radau shape coordinates, provides a much more perfect separation of rotations and vibrations than the so-called $r_{1}$ or $r_{2}$ embeddings.

It was Eckart (Eckart, 1935) who formulated equations that lead to a good separation of rotations and vibrations, yielding exactly zero rotational-vibrational coupling at a chosen reference structure (actually this is how the Eckart embedding is defined). The optimal separation of the rotational and vibrational dofs is often crucial for obtaining converged rovibrational energy levels and eigenstates in practical nuclear-motion computations. Other possible motivations for using the Eckart frame include the assignment of rovibrational eigenstates (Mátyus et al., 2010, Szidarovszky et al., 2012), the computation of vibrational band intensities (Le Sueur et al., 1992), and minimizing the root-mean-square deviation between two molecular conformations (Kudin and Dymarsky, 2005).

The translational Eckart condition,

$$
\sum_{i=1}^{N} m_{i} \mathbf{x}_{i}=\mathbf{0},
$$

where the vectors $\mathbf{x}_{i}$ denote the instantaneous position vectors of the $N$ nuclei in the Eckart frame, is supplemented with the rotational Eckart condition,

$$
\sum_{i=1}^{N} m_{i}\left(\mathbf{x}_{i} \times \mathbf{a}_{i}\right)=\mathbf{0},
$$


where the vectors $\mathbf{a}_{i}$ specify the reference configuration, whereby the rovibrational coupling vanishes. The translational Eckart condition can be satisfied by placing the origin of the bodyfixed frame at the center of mass, while fulfilling the rotational Eckart condition is equivalent to finding a $\mathbf{T}$ (pseudo)rotation matrix which transforms the $\mathbf{x}_{i}^{\prime}$ initial position vectors into the $\mathbf{x}_{i}=\mathbf{T} \mathbf{x}_{i}^{\prime}$ position vectors corresponding to the Eckart frame. Following Eckart (1935), Strauss and Pickett (1970) devised a procedure for finding the $\mathbf{T}$ transformation matrix. An important shortcoming of the methods of Eckart (1935) and of Strauss and Pickett (1970) is the need for computing the inverse of a matrix which can be singular for certain nuclear configurations. Two new methods, achieving the same task but free of the singularity problem, have been developed recently (Dymarsky and Kudin, 2005, Krasnoshchekov et al., 2014).

As the Eckart frame is crucial to obtain good separation of vibrations and rotations, there has been a considerable effort to derive Eckart-embedded KEOs. For rectilinear vibrational coordinates, including normal coordinates, the theory has been worked out by Watson (Watson, 1968, 1970). For curvilinear internal coordinates, analytic Eckart-embedded KEOs have been derived for the limiting class of triatomic (Wei and Carrington Jr., 1997, Wei and Carrington, 1997, Wei and Carrington Jr., 1998) as well as the more general class of planar molecules (Wei, 2003a,b). Unfortunately, the Eckart-embedded KEOs expressed in curvilinear coordinates have rather bulky forms preventing their widespread use. It is worth noting at this point that flexible reference configurations, as opposed to the choice of a rigid, e.g., equilibrium structure, have been introduced in several spectroscopic models, including the Hougen-Bunker-Johns approach (Hougen et al., 1970) and several of its extensions and variants (Špirko, 1983, Szalay, 1988, Yurchenko et al., 2005).

The challenge to construct general analytic Eckart-embedded KEOs expressed in arbitrary curvilinear coordinates for arbitrary molecules can be circumvented if the KEO is treated numerically (Luckhaus, 2000, Lauvergnat and Nauts, 2002, Yurchenko et al., 2007, Mátyus et al., 2009, Strobusch and Scheurer, 2011a,b, Fábri et al., 2011a). This can be achieved in grid-based codes (Fábri et al., 2014b) by transforming the body-fixed position vectors $\mathbf{x}_{i}$ to the Eckart frame using the quaternion-based transformation method outlined in (Krasnoshchekov et al., 2014) and then evaluating the $\frac{\partial \mathbf{x}_{i}}{\partial q_{k}}$ vectors [see Eq. (14)] by numerical differentiation of the Eckart-embedded $\mathbf{x}_{i}$ vectors (Szidarovszky et al., 2012, Fábri et al., 2014b), while the computation of the $\mathbf{u}_{k} \times \mathbf{x}_{i}$ vectors used in Eqs. (12) and (15) simply require the Eckart-embedded $\mathbf{x}_{i}$ vectors (Szidarovszky et al., 2012, Fábri et al., 2014b). This allows the user to employ the Eckart frame for arbitrary vibrational coordinates with either fixed or flexible reference structures. Other approaches for the application of the Eckart frame have also been reported (McCoy et al., 1991, Wang and Carrington Jr., 2013, Sadri et al., 2014, Pesonen, 2014, Szalay, 2014, 2015a,b, Yachmenev and Yurchenko, 2015, Szalay, 2017).

\subsection{Potential energy hypersurfaces}

The concept of a PES (Murrell et al., 1984, Mezey, 1987, Császár et al., 2001, Marquardt and Quack, 2011) is fundamental to the understanding of most modern fields of chemistry, PESs are especially relevant for studies in high-resolution spectroscopy, detailed chemical kinetics, and nuclear dynamics. For an arbitrary chemical system, PESs describe the variation of the total electronic energy as a function of the (relative) nuclear positions. In computational molecular 
spectroscopy it is still usual to focus on cases where a single BO-PES is sufficiently uncoupled from the other surfaces (electronic states) that their interaction may safely be ignored. This is usually true for the PES characterizing the lowest (ground) electronic state, as the error thus introduced is smaller than the intrinsic error of the PES. In dynamical studies it is more usual to couple a few selected PESs, perhaps just two, and define adiabatic and diabatic representations (Smith, 1969, Song and Gao, 2008).

Although PESs developed for rovibrational spectroscopy, chemical reactions, and intermolecular interactions representing systems of non-covalent chemistry have a lot in common, there are many important differences in their construction. These differences originate from the fact that these surfaces need to concentrate on different configuration regions where different electronicstructure techniques are suitable, and that they aim to interpret different types of experimental information of widely different accuracy. The four distinct types of (global) PESs available in the literature are empirical, semiempirical, semitheoretical, and theoretical. Empirical potentials are rather inaccurate even for triatomic systems and they are basically unavailable for larger ones. As to theoretical PESs, due to well recognized inadequacies of most electronic structure computations (Császár et al., 2001), it is still not possible to even approach the accuracy required by high-resolution spectroscopic measurements, except perhaps for the smallest systems (vide infra). Even today, the highest quality PESs are obtained by a combination of theoretical and experimental approaches.

Excellent books (Murrell et al., 1984, Mezey, 1987) and reviews (Császár et al., 2001, 2000, Majumder et al., 2016) are available discussing the generation and utilization of local as well as global PESs; thus, they are not discussed in detail here. We are only mentioning a couple of recent developments worth emphasizing.

The $n$-mode (or multimode) representation of the PES (Carter et al., 1997a,b, Bowman et al., 2003, Rauhut, 2004, Sibaev and Crittenden, 2016, Ziegler and Rauhut, 2016) is highly useful for several types of nuclear-motion applications, especially those employing the EW Hamiltonians. These PESs may prove less useful when large configuration regions need to be sampled. The use of permutationally invariant PESs (Braams and Bowman, 2009) is particularly advantageous for systems containing more than 4-5 atoms and this concept leads to an almost automatic generation of a "global" PES. Almost automatic generation of the PES is highly desirable; thus, use of neural networks and artificial intelligence have been explored (Handley and Popelier, 2010, Majumder et al., 2015, Jiang et al., 2016, Petty et al., 2018) for this purpose. For chemical reactions proper representation of the surface close to an intrinsic reaction path (IRP) (Fukui, 1981), a minimum-energy path (MEP) (Fukui, 1970), or some other path (Müller, 1980) is often what is used to determine the location of the points underlying the PES (Klepeis et al., 1993). Stationary points (beyond minima and first-order transition states) characterizing the given PES form are rarely reported, not even for molecular complexes, though they may serve important qualitative and quantitative purposes.

\subsection{Basis sets and representations}

Basis sets and the representation of $\hat{H}^{\text {int }}$ in the chosen basis play an extremely important role in the effectiveness of the variational approaches characterizing nuclear-motion theory. Thus, it 
is not surprising that a considerable number of efficient numerical techniques have been developed, with a concerted effort to tailor the basis sets and the representations to the usual need of computing an exceedingly large number of rovibrational eigenstates.

Today it appears that the solution of the time-independent rovibrational Schrödinger equation is achieved perhaps most efficiently by grid-based techniques. The utility and the basic aspects of grid-based techniques were realized by different groups working in different fields independently and thus several somewhat distinct methods were developed, including the discrete variable representation (DVR) (Harris et al., 1965, Dickinson and Certain, 1968, Lill et al., 1982, Light et al., 1985, Light and Carrington Jr., 2000), the Lagrange-mesh (Baye and Heenen, 1986), the quadrature discretization (Shizgal and Blackmore, 1984, Blackmore and Shizgal, 1985), and the Fourier grid (Kosloff and Kosloff, 1983, Marston and Bálint-Kürti, 1989, Layton, 1993) methods. Of these we will concentrate here only on the DVR technique.

When the Hamiltonian is represented by a DVR, one basically employs both a set of basis functions and a set of grid points. Within the DVR and finite basis representation (FBR) methods the operators of the physical quantities considered are represented by matrices with indices referring either to grid points or spectral basis functions, respectively. The DVR of the potential energy operator is extremely simple, a diagonal matrix with the $(n n)$ th diagonal element equal to the value of the PES function taken at the $n$th grid point. The FBR of the potential energy operator corresponds to evaluating integrals in the spectral basis by numerical quadrature, whereby the quadrature grid is identical to the one defining the DVR. The DVR and FBR of an operator of a physical quantity are related by a unitary transformation.

At the beginning (Harris et al., 1965, Dickinson and Certain, 1968), the DVR developed was based on standard orthogonal polynomial bases and the associated Gaussian quadratures. Within this simplest DVR scheme the same number of basis functions and quadrature points are employed. Later, generalizations of the DVR method to more general basis functions depending on a single variable and to multidimensional nondirect product basis sets have been developed (Light et al., 1985, Kanfer and Shapiro, 1984, Corey and Tromp, 1995, Szalay, 1996, Littlejohn et al., 2002, Szalay et al., 2003, Dawes and Carrington Jr., 2004, Yu, 2005). A family of generalized DVR (GDVR) techniques, including the optimal GDVR, was introduced by Szalay (Szalay, 1996). Similar to the relation between DVR and FBR schemes, GDVR techniques have a strong connection with generalized FBR (GFBR) techniques (Szalay, 1996, Czakó et al., 2006). In a GFBR one is allowed to use more grid points than basis functions, leading to an improved accuracy of the results. Consideration of boundary conditions and singularities (Szalay et al., 2012) is extremely important for the application of all flavors of FBR and DVR techniques. This becomes especially important when one does not know the actual form of the kinetic energy operator as it is used just numerically on a grid. A particularly important characteristic of DVR bases is that they provide numerically exact solution of the time-independent rovibrational Schrödinger equation without computing the Hamiltonian matrix elements exactly (Szalay et al., 2012). For this reason, techniques based on DVR should not be considered strictly variational.

There are different strategies toward selecting the most useful and practical form of the rovibrational Hamiltonian. One possible strategy is to define lower-dimensional but non-sparse and non-direct-product matrices. Contraction of the vibrational basis (Carter and Handy, 1988, Bačić and Light, 1989) offers considerable numerical advantages, while other strategies, based 
on direct-product bases, produce often enormous-dimensional but sparse matrices with a special structure. There is not much choice how rotational basis functions can be chosen. A very practical and efficient choice of a rotational basis involves Wang functions (Zare, 1988), as discussed in Section 3.1. Naturally, different "diagonalization" techniques are required for the different Hamiltonians (see Section 2.7). Some further details, relevant to this chapter, will be given in Section 3.

\subsection{Determination of eigenstates}

Each variational technique, at least in principle, requires the diagonalization of the Hamiltonian yielding rovibrational eigenvalues and eigenvectors. In order to compute the required many (often tens of thousands of) eigenpairs for medium-sized molecules, an iterative eigensolver [the usual choice is the Lanczos technique (Lanczos, 1950, Cullum and Willoughby, 1985)] must be implemented, adapted to the features and requirements of nuclear-motion theory (Wang and Carrington Jr., 2001, Mátyus et al., 2009).

The conventional iterative Lanczos algorithm (Lanczos, 1950) converges to the dominant eigenvalues of the matrix. The convergence rate of the Lanczos iteration is largely determined by the relative separation of the eigenvalues. To compute the lowest or interior eigenvalues and speed up convergence, in variational nuclear-motion theory one needs to introduce spectral transformation techniques (Ericsson and Ruhe, 1980, Kono, 1993, Wyatt, 1995). Thus, families of polynomial transformation techniques (Sorensen, 1992, Wang and Zunger, 1994), exponential filters using a Chebyshev expansion (Yu and Nyman, 1998, 1999), and shift-invert (Ericsson and Ruhe, 1980, Mátyus et al., 2009) filtering techniques using the conjugate gradient method (CGM) have been introduced. Nevertheless, construction of an efficient black-box Lanczos variant, for instance, a method that is efficient for any spectral range of a rotation-vibration Hamiltonian, remains a challenge. In each Lanczos step the original matrix is transformed, requiring a certain number of matrix-vector multiplications. These multiplications form the most CPU-intensive part of the computation; thus, the number of matrix-vector multiplications required determines the "cost" of a specific spectral transformation. The spectral properties of matrices corresponding to different spectral transformation methods can be very different. This is an important consideration as the relative separation of the eigenvalues of the matrix introduced in the Lanczos iteration influences strongly the convergence rate of the iteration.

Under exact arithmetics, the Lanczos vectors are orthogonal by construction (Saad, 2003). In the presence of computational round-off errors, this orthogonality is not maintained. The loss of orthogonality manifests itself in the appearance of (a) spurious eigenvalues, and (b) copies of correct ones. To remove the extra and spurious eigenvalues a posteriori, Cullum and Willoughby (Cullum and Willoughby, 1985) as well as Wang and Carrington (Wang and Carrington Jr., 2002) suggested useful and relatively inexpensive algorithms. A somewhat expensive alternative is to maintain (semi)orthogonality among the Lanczos vectors throughout the iterations. It has also been demonstrated (Simon, 1984, Wu and Simon, 1998, Mátyus et al., 2009) that maintaining full orthogonality is not necessary and semiorthogonality of Lanczos vectors, provided by partial reorthogonalization, is sufficient, accurate eigenpairs without extra and spurious levels entering the spectrum can be computed this way. To keep storage requirements under control, the Lanczos algorithm must be occasionally restarted (Wu and Simon, 
1998, Wu et al., 1999, Mátyus et al., 2009).

\section{Computation of bound rovibrational states}

There are three distinct classes of methods for the variational solution of the time-independent nuclear Schrödinger equation,

$$
\hat{H}^{\mathrm{int}} \Psi_{i}=E_{i} \Psi_{i},
$$

based on Hamiltonians detailed in Section 2: (a) the first one is based on tailor-made Hamiltonians, with explicitly derived kinetic energy operators expressed in terms of orthogonal or nonorthogonal internal coordinates (Shipsey, 1998, Bramley et al., 1991, Chapuisat and Iung, 1992, Klepeis et al., 1993, Henderson and Tennyson, 1993, Schwenke, 1996, Mladenović, 2000a,b,c, Czakó et al., 2004, Tennyson et al., 2004, Kozin et al., 2004, Huang et al., 2008, Szidarovszky et al., 2010); (b) the second one utilizes the EW Hamiltonians, relying on a single reference structure, rectilinear coordinates, and the Eckart embedding (Carter et al., 1998, Tew et al., 2001, Bowman et al., 2003, Mátyus et al., 2007, 2009); and (c) the third one uses arbitrary vibrational (shape) coordinates preferably supplemented with the numerical construction of the kinetic-energy operator (Lauvergnat and Nauts, 2002, Yurchenko et al., 2007, Mátyus et al., 2009, Sadri et al., 2014, Changala, 2019), so that the exact form of the kinetic-energy operator does not need to be known a priori.

Tailor-made Hamiltonians have been employed extensively (Sutcliffe and Tennyson, 1991, Klepeis et al., 1993, Tennyson et al., 2004, Kozin et al., 2004, Szidarovszky et al., 2010). As noted more than three decades ago, the "disadvantage with [tailor-made Hamiltonians] is that a separate program has to be developed for each molecule and for each chosen coordinate system" (Handy, 1987). Therefore, codes belonging to this class cannot be considered as "black-box" approaches to variational nuclear-motion computations for polyatomic molecules. For this reason this class of approaches is not discussed here further.

There are a number of codes available which are based on the use of the EW Hamiltonians (Bowman et al., 2003, Mátyus et al., 2007, Christiansen, 2007, Neff and Rauhut, 2009). These codes are completely general, can be employed for rather large systems, especially after the introduction of special sophisticated algorithms. Nevertheless, they cannot be applied to complex nuclear motions and for molecules exhibiting several LAMs. Therefore, their discussion is terminated here, the interested reader is referred to the extensive literature (Bowman et al., 2003, Mátyus et al., 2007) on their form and use. From here on only approach (c) from above is considered.

\subsection{On the variational solution}

To compute bound rovibrational states variationally, the matrix representation of $\hat{H}^{\text {int }}$ needs to be considered. For this, it is advantageous to split $\hat{T}^{\text {int }}$ into three terms:

$$
\hat{H}^{\mathrm{int}}=\hat{T}^{\mathrm{int}}+\hat{V}=\hat{T}^{\mathrm{v}}+\hat{T}^{\mathrm{r}}+\hat{T}^{\mathrm{rv}}+\hat{V},
$$


where (see Eq. (16))

$$
\begin{gathered}
\hat{T}^{\mathrm{v}}=\frac{1}{2} \sum_{k=1}^{A_{\mathrm{s}}} \sum_{l=1}^{A_{\mathrm{s}}} \tilde{g}^{-1 / 4} \hat{p}_{k}^{\dagger} G^{k l} \tilde{g}^{1 / 2} \hat{p}_{l} \tilde{g}^{-1 / 4}, \\
\hat{T}^{\mathrm{r}}=\frac{1}{2} \sum_{k=1}^{3} G^{k+A_{s}, k+A_{\mathrm{s}}} \hat{J}_{k}^{2}+\frac{1}{2} \sum_{k=1}^{3} \sum_{l>k}^{3} G^{k+A_{\mathrm{s}}, l+A_{\mathrm{s}}}\left[\hat{J}_{k}, \hat{J}_{l}\right]_{+},
\end{gathered}
$$

and

$$
\hat{T}^{\mathrm{rv}}=\frac{1}{2} \sum_{l=1}^{3} \sum_{k=1}^{A_{\mathrm{s}}}\left(\hat{p}_{k}^{\dagger} G^{k, l+A_{\mathrm{s}}}+G^{k, l+A_{\mathrm{s}}} \hat{p}_{k}\right) \hat{J}_{l} .
$$

In Eqs. (23)-(25), $\hat{T}^{\mathrm{v}}$ and $\hat{T}^{\mathrm{r}}$ are the vibrational and rotational kinetic energy operator parts, respectively, and $\hat{T}^{\text {rv }}$ gives the coupling between vibrations and rotations (often referred to as the Coriolis term). In Eq. (24), $\hat{J}_{k}$ is the $k$ th body-fixed component of $\hat{\mathbf{J}}$ and $\left[\hat{J}_{k}, \hat{J}_{l}\right]_{+}$refers to the anticommutator of $\hat{J}_{k}$ and $\hat{J}_{l}$. As the $\hat{J}_{k}$ angular momentum components correspond to the body-fixed frame, they satisfy the anomalous commutation relations (Zare, 1988)

$$
\left[\hat{J}_{k}, \hat{J}_{l}\right]=-\mathrm{i} \epsilon_{k l m} \hat{J}_{m},
$$

where $\epsilon_{k l m}$ is the Levi-Cività permutation symbol, and the Einstein summation convention is applied.

The multidimensional vibrational basis functions can be constructed as the direct product of one-dimensional primitive or potential-optimized (PO) (Echave and Clary, 1992, Wei and Carrington Jr., 1992, Szalay et al., 2003) DVR functions,

$$
F_{i}(\mathbf{q})=\prod_{\alpha=1}^{A_{\mathrm{s}}} f_{i_{\alpha}}\left(q_{\alpha}\right)
$$

where $f_{i_{\alpha}}\left(q_{\alpha}\right)$ denote a 1D DVR function associated with vibrational dof $\alpha$ and 1D DVR grid point $q_{i_{\alpha}}^{(\alpha)}$. This choice assures that the coordinate-dependent $G^{k l}, \tilde{g}$, and $\hat{V}$ operators are represented by diagonal matrices in the direct-product DVR basis. Moreover, the diagonal matrix elements

$$
\left\langle F_{i}|\hat{O}| F_{j}\right\rangle=O\left(q_{i_{1}}^{(1)}, \ldots, q_{i_{A_{\mathrm{s}}}}^{\left(A_{\mathrm{s}}\right)}\right) \prod_{\alpha=1}^{A_{\mathrm{s}}} \delta_{i_{\alpha}, j_{\alpha}}
$$

are equal to the values of the given coordinate-dependent operator $\hat{O}$ evaluated at the DVR grid points. Therefore, the $\mathbf{t}$ vectors needed to construct $\hat{T}^{\text {int }}$ have to be evaluated at the DVR grid points, which can be done either analytically or numerically for a specific choice of internal coordinates and body-fixed frame embeddings. This is what makes the numerical construction of $\hat{T}^{\text {int }}$ possible and obviates the need for tailor-made kinetic-energy operators. Matrix elements of the vibrational $\hat{p}_{k}$ operators can be expressed as

$$
\left\langle F_{i}\left|\hat{p}_{k}\right| F_{j}\right\rangle=\left\langle f_{i_{k}}\left(q_{k}\right)\left|\hat{p}_{k}\right| f_{j_{k}}\left(q_{k}\right)\right\rangle \prod_{\alpha=1, \alpha \neq k}^{A_{\mathrm{s}}} \delta_{i_{\alpha}, j_{\alpha}},
$$

where $\left\langle f_{i_{k}}\left(q_{k}\right)\left|\hat{p}_{k}\right| f_{j_{k}}\left(q_{k}\right)\right\rangle$ is proportional to the corresponding matrix element of the first derivative operator $\frac{\partial}{\partial q_{k}}$ in the DVR basis applied (Szalay, 1993). 
For a given rotational angular momentum quantum number $J$ (note that the molecular system is isolated and no external fields are present), the set of $2 J+1$ orthonormal $|J K M\rangle$ symmetric rigid-rotor eigenfunctions serve as a suitable basis to set up the matrix representation of $\hat{H}^{\text {int }}$. According to Eqs. (24) and (25), the matrix representation of $\hat{J}_{k}, \hat{J}_{k}^{2}$ and $\left[\hat{J}_{k}, \hat{J}_{l}\right]_{+}$is required to solve the rovibrational problem. The complete set of nonzero $\hat{J}_{k}$ matrix elements (Zare, 1988) is given by

$$
\begin{gathered}
\left\langle J K M\left|\hat{J}_{x}\right| J(K \pm 1) M\right\rangle=\frac{1}{2} \sqrt{J(J+1)-K(K \pm 1)}, \\
\left\langle J K M\left|\hat{J}_{y}\right| J(K \pm 1) M\right\rangle=\mp \frac{\mathrm{i}}{2} \sqrt{J(J+1)-K(K \pm 1)}, \\
\left\langle J K M\left|\hat{J}_{z}\right| J K M\right\rangle=K,
\end{gathered}
$$

where $K=-J, \ldots, J$ corresponds to the body-fixed $z$, while $M=-J, \ldots, J$ to the space-fixed $Z$ components of the overall angular momentum. The $\hat{J}_{k}^{2}$ and $\left[\hat{J}_{k}, \hat{J}_{l}\right]_{+}$matrices can be computed exactly by inserting the resolution of identity between $\hat{J}_{k}$ and $\hat{J}_{l}$, and thus

$$
\left\langle J K M\left|\hat{J}_{k} \hat{J}_{l}\right| J K^{\prime} M\right\rangle=\sum_{K^{\prime \prime}=-J}^{J}\left\langle J K M\left|\hat{J}_{k}\right| J K^{\prime \prime} M\right\rangle\left\langle J K^{\prime \prime} M\left|\hat{J}_{l}\right| J K^{\prime} M\right\rangle .
$$

In practice, a good choice for the rotational basis involves the Wang functions that yield real Hamiltonian matrix elements and transform according to the irreducible representations of the $D_{2}$ rotational group (Zare, 1988).

Construction of $\mathbf{H}^{\text {int }}$ requires the introduction of a rovibrational basis. For this purpose one can employ the direct product of the multidimensional direct-product DVR vibrational basis functions and the $2 J+1$ Wang rotational basis functions. Using Eqs. (22)-(25), $\mathbf{H}^{\text {int }}$ takes the form

$$
\mathbf{H}^{\text {int }}=\mathbf{T}^{\text {int }}+\mathbf{V}=\mathbf{T}^{\mathrm{v}}+\mathbf{T}^{\mathrm{r}}+\mathbf{T}^{\mathrm{rv}}+\mathbf{V},
$$

where

$$
\begin{gathered}
\mathbf{T}^{\mathrm{v}}=\frac{1}{2} \mathbf{I}_{2 J+1} \otimes \sum_{k=1}^{A_{\mathrm{s}}} \sum_{l=1}^{A_{\mathrm{s}}} \tilde{\mathbf{g}}^{-1 / 4} \mathbf{p}_{k}^{\dagger} \mathbf{G}^{k l} \tilde{\mathbf{g}}^{1 / 2} \mathbf{p}_{l} \tilde{\mathbf{g}}^{-1 / 4}, \\
\mathbf{T}^{\mathrm{r}}=\frac{1}{2} \sum_{k=1}^{3} \mathbf{J}_{k}^{2} \otimes \mathbf{G}^{k+A_{\mathrm{s}}, k+A_{\mathrm{s}}}+\frac{1}{2} \sum_{k=1}^{3} \sum_{l>k}^{3}\left[\mathbf{J}_{k}, \mathbf{J}_{l}\right]_{+} \otimes \mathbf{G}^{k+A_{\mathrm{s}}, l+A_{\mathrm{s}}}, \\
\mathbf{T}^{\mathrm{rv}}=\frac{1}{2} \sum_{l=1}^{3} \mathbf{J}_{l} \otimes \sum_{k=1}^{A_{\mathrm{s}}}\left(\mathbf{p}_{k}^{\dagger} \mathbf{G}^{k, l+A_{\mathrm{s}}}+\mathbf{G}^{k, l+A_{\mathrm{s}}} \mathbf{p}_{k}\right),
\end{gathered}
$$

and

$$
\mathbf{V}=\mathbf{I}_{2 J+1} \otimes \mathbf{V}_{J=0},
$$

where $\mathbf{I}_{2 J+1}$ is the identity matrix of dimension $2 J+1, \otimes$ refers to the direct product operation, $\mathbf{V}_{J=0}$ denotes the diagonal potential-energy matrix in the direct-product DVR vibrational basis, and multiple resolutions of identity have been inserted in between some of the neighboring operators.

The iterative Lanczos algorithm (Lanczos, 1950, Cullum and Willoughby, 1985) can be utilized to compute the required eigenvalues and eigenvectors of $\mathbf{H}^{\text {int }}$. Then one must evaluate 
the effect of $\mathbf{H}^{\text {int }}$ on an arbitrary vector of the same dimension. As $\mathbf{H}^{\text {int }}$ has a special and very sparse structure, the explicit construction and storage of $\mathbf{H}^{\text {int }}$ can be avoided during the computation. Instead, one has to implement the $\mathbf{H}^{\text {int }} \mathbf{x}$ matrix-vector multiplication, which can be done efficiently due to the special sparse structure of $\mathbf{H}^{\text {int }}$ (Mátyus et al., 2009, Fábri et al., 2011a).

An alternative solution of the rovibrational problem is provided by the vibrational subspace (VS) method (Fábri et al., 2011b, 2019) relying on the

$$
\hat{H}^{\mathrm{int}}=\hat{H}^{\mathrm{v}}+\hat{T}^{\mathrm{r}}+\hat{T}^{\mathrm{rv}}
$$

partition of $\hat{H}^{\text {int }}$, where $\hat{H}^{\mathrm{v}}=\hat{T}^{\mathrm{v}}+\hat{V}$ refers to the vibrational Hamiltonian. After solving the

$$
\hat{H}^{\mathrm{v}} \Phi_{j}=E_{j} \Phi_{j}
$$

vibrational Schrödinger equation and obtaining the $E_{j}$ vibrational energy levels and $\Phi_{j}$ vibrational eigenstates, the rovibrational eigenstates can be expanded in the form

$$
\Psi_{i}=\sum_{j=1}^{n} \sum_{k=1}^{2 J+1} c_{j k}^{(i)} \Phi_{j} R_{k},
$$

where $R_{k}$ denotes rotational basis functions (symmetric top eigenfunctions, Wang functions, or rigid rotor eigenfunctions of the molecule under investigation). The VS ansatz of Eq. (39) offers a compact representation of the rovibrational eigenstates as the size of the VS rovibrational Hamiltonian remains moderate compared to the size of the DVR Hamiltonian even for high $J$ values. We found (Fábri et al., 2011b) that it is inevitable to apply the Eckart frame in conjunction with the VS method to achieve satisfactory convergence of the computed rovibrational energy levels and eigenstates.

Finally, note that it is possible to simplify $\hat{T}^{\mathrm{v}}$ of Eq. (23) at the cost of adding the so-called extrapotential term

$$
\hat{U}=\frac{1}{32} \sum_{k=1}^{A_{\mathrm{s}}} \sum_{l=1}^{A_{\mathrm{s}}}\left[\frac{G^{k l}}{\tilde{g}^{2}} \frac{\partial \tilde{g}}{\partial q_{k}} \frac{\partial \tilde{g}}{\partial q_{l}}+4 \frac{\partial}{\partial q_{k}}\left(\frac{G^{k l}}{\tilde{g}} \frac{\partial \tilde{g}}{\partial q_{l}}\right)\right]
$$

to the potential energy. The so-called rearranged vibrational Hamiltonian becomes

$$
\hat{H}^{\mathrm{v}, \text { rearr }}=\frac{1}{2} \sum_{k=1}^{A_{\mathrm{s}}} \sum_{l=1}^{A_{\mathrm{s}}} \hat{p}_{k}^{\dagger} G^{k l} \hat{p}_{l}+\hat{U}+\hat{V},
$$

while the operators $\hat{T}^{\mathrm{r}}$ and $\hat{T}^{\mathrm{rv}}$ do not change. Although the vibrational kinetic energy operator is simplified with the introduction of $\hat{U}$, the rearranged form is more prone to numerical instabilities than the so-called Podolsky form defined in Eq. (16); therefore, the Podolsky form is preferred over the rearranged form in practical applications (Mátyus et al., 2009).

\subsection{Symmetry in nuclear-motion computations}

The symmetry group of $\hat{H}^{\text {int }}$ contains all symmetry operations that commute with $\hat{H}^{\text {int }}$. These symmetry operations are the permutations of identical nuclei and the operation of space inversion, and they form the complete nuclear permutation inversion (CNPI) group (Bunker and 
Jensen, 1998), while the symmetry group constituted by the so-called feasible permutationinversion symmetry elements is called the molecular symmetry (MS) group, $G=\left\{g_{i} \mid i=\right.$ $1, \ldots,|G|\}$, where $|G|$ denotes the order of $G$ (Longuet-Higgins, 1963, Quack, 1977, Bunker and Jensen, 1998, Mills and Quack, 2002, Quack, 2011, Schnell, 2011, Oka, 2011).

As $\left[g_{i}, \hat{H}^{\mathrm{int}}\right]=0$, the $n$-fold degenerate $\psi_{i j}$ eigenfunctions corresponding to the energy eigenvalue $E_{i}$ span an $n$-dimensional irreducible representation of $G$. According to the grand orthogonality theorem (GOT) (Wigner, 1959), basis functions spanning the $\Gamma_{\alpha}$ irreducible representation can be constructed by the projector

$$
P_{\alpha j}=\frac{n_{\alpha}}{|G|} \sum_{i=1}^{|G|} D_{j j}^{\alpha^{*}}\left(g_{i}\right) g_{i},
$$

where the dimension of the irreducible representation $\Gamma_{\alpha}$ is $n_{\alpha}, j=1, \ldots, n_{\alpha}$, and $\mathbf{D}^{\alpha}\left(g_{i}\right)$ is the matrix representation of $g_{i}$ in $\Gamma_{\alpha}$. Although $P_{\alpha j}$ commutes with $\hat{H}^{\text {int }}$, in practical computations the matrix representations of certain symmetry operations may not commute with the Hamiltonian matrix due to numerical errors (Wang and Carrington, 2003, Poirier, 2003a, Wang and Carrington Jr., 2003a, 2005). In this section we provide a brief account of the implementation of molecular symmetry in a DVR-based code (Fábri et al., 2017). For alternative approaches we refer the reader to the literature (Wang and Carrington Jr., 2001, Wang and Carrington, 2003, Poirier, 2003a, Wang and Carrington Jr., 2003a,b, 2004, 2005, Yurchenko et al., 2017).

In order to obtain symmetry-adapted linear combinations of the grid-based vibrational basis functions, $P_{\alpha j}$ must act on direct-product DVR functions denoted by $F_{k}$. We require that each direct-product DVR function (or equivalently the associated multidimensional grid point) is mapped onto another DVR function (or onto itself in special cases) by any of the $g_{i}$ symmetry operations. The symmetry-adapted vibrational basis functions

$$
\chi_{i}^{\alpha}=\sum_{k} A_{i k}^{\alpha} F_{k}
$$

can be expressed as the linear combination of direct-product DVR functions.

As the iterative Lanczos algorithm is used usually to diagonalize the vibrational Hamiltonian, we need to devise a procedure to evaluate matrix-vector products

$$
y_{i}^{\alpha}=\sum_{j}\left\langle\chi_{i}^{\alpha}\left|\hat{H}^{\mathrm{int}}\right| \chi_{j}^{\alpha}\right\rangle x_{j}^{\alpha}=\sum_{k} A_{i k}^{\alpha^{*}} \sum_{l}\left\langle F_{k}\left|\hat{H}^{\mathrm{int}}\right| F_{l}\right\rangle \sum_{j} A_{j l}^{\alpha} x_{j}^{\alpha}
$$

in the symmetry-adapted vibrational basis. First, a symmetrized input vector $\mathbf{x}^{\alpha}$ from $\Gamma_{\alpha}$ is transformed to the original unsymmetrized vibrational basis (sum over $j$ ), then the resulting fulldimensional vector is multiplied by the unsymmetrized Hamiltonian (sum over $l$ ), and, finally, the full-dimensional result vector is transformed back to the symmetry-adapted basis (sum over $k$ ), yielding the symmetry-adapted output vector $\mathbf{y}^{\alpha}$. Thus, while the dimension of the input $\mathbf{x}$ and output $\mathbf{y}$ vectors is reduced due to symmetry, the second step involves a multiplication with the Hamiltonian represented in the unsymmetrized vibrational basis. Although it is possible to evaluate matrix-vector products directly in the symmetry-adapted basis, we have found it more convenient to apply the matrix-vector product algorithm outlined in Eq. (44), also related to the symmetry-adapted Lanczos method (Wang and Carrington Jr., 2001, Wang and Carrington, 
2003, Poirier, 2003a, Wang and Carrington Jr., 2003a,b, 2004, 2005). The symmetry-adapted computation provides degenerate energy levels only once, since projectors $P_{\alpha j}$ are applied with a single $j$ index. This implies that we get only one eigenstate $\left(\psi_{i j}\right)$ for each degenerate manifold of dimension $n$. The missing $n-1$ eigenstates can be obtained by applying symmetry operations on $\psi_{i j}$ and generating an orthonormal basis from the $g_{k} \psi_{i j}$ functions, similar to Wang and Carrington Jr. (2008).

It is important to emphasize that the symmetrization algorithm presented is completely general and has the following advantages over the unsymmetrized treatment: (a) the dimension of the Lanczos vectors spanning the so-called Krylov subspace (Lanczos, 1950, Cullum and Willoughby, 1985) is reduced, therefore the memory requirement is reduced; (b) the computed energy levels and eigenstates are automatically labeled with $\Gamma_{\alpha} ;$ (c) as the convergence rate of the Lanczos algorithm deteriorates with the increasing density of the energy levels, it is advantageous to decrease the spectral density of the Hamiltonian by separating the eigenstates according to symmetry. The only limitation of the vibrational symmetrization approach outlined is that the image of each direct-product grid point generated by any kind of symmetry operation has to be an element of the multidimensional DVR grid. Unfortunately, this requirement may limit the practically applicable symmetry group to a subgroup of the full MS group. One such case is that of the $\mathrm{CH}_{5}^{+}$molecule, described in Section 5.

\subsection{Nuclear spin statistics}

Knowledge of the nuclear spin (ns) statistical weights (Landau and Lifshitz, 1977, Bunker and Jensen, 1998) of rovibronic (in the simplest case rovibrational) eigenstates of a molecule is necessary for a detailed understanding of molecular high-resolution spectra for at least two important reasons: (a) only levels with a nonzero ns statistical weight will be involved in measured transitions, and (b) the intensities of measured lines is directly related to the ns statistical weights. The interaction of nuclear-spin angular momenta with other angular momenta characterizing the molecule is extremely weak; thus, it is an excellent approximation to separate the total molecular wavefunction into nuclear spin and rotation-vibration-electronic-electron-spin parts:

$$
\left|\Psi^{\text {total }}\right\rangle=\left|\Psi^{\mathrm{ns}}\right\rangle \otimes\left|\Psi^{\mathrm{r}, \mathrm{v}, \mathrm{e}, \mathrm{es}}\right\rangle .
$$

Knowing the symmetry of $\left|\Psi^{\text {total }}\right\rangle$ and $\left|\Psi^{\text {ns }}\right\rangle$ restricts the symmetry of $\left|\Psi^{\mathrm{r}, \mathrm{v}, \mathrm{e}, \mathrm{es}}\right\rangle$. The nuclear spin statistics, and consequently the degeneracy factors also depend on the nuclear spin $I$ of the nonpermuting nuclei. The ns statistical weights can be determined via different methods (Landau and Lifshitz, 1977, Bunker and Jensen, 1998).

Molecules containing identical nuclei with non-zero nuclear spins, $I$, can exist as more than one nuclear-spin isomers. An old example is the $\mathrm{H}_{2}$ molecule (Bonhoeffer and Harteck, 1929), with two spin-1/2 H nuclei, it can exist in ortho $(I=1)$ and para $(I=0)$ forms. para- $\mathrm{H}_{2}$, once prepared (Bonhoeffer and Harteck, 1929), can exist in an appropriate container at room temperature for months. Polyatomic molecules may have more nuclear-spin isomers than $\mathrm{H}_{2}$. $\mathrm{CH}_{4}$, for example, has ortho $(I=1)$, meta $(I=2)$, and para $(I=0)$ forms. Larger molecules may have even more nuclear-spin isomers.

Related to nuclear-spin isomers and the related states one must note an important peculiarity 
of time-independent variational nuclear-motion computations: they tend to yield eigenstates which may not exist in nature due to the Pauli exclusion principle (Herzberg, 1945). This is the result of not considering nuclear spin explicitly during the standard variational rovibrational computations (this is advantageous as this way the same code, the same BO-PES, and the same variational parameters can be used for nuclear-motion computations involving different isotopologues and isotopomers). Thus, computed rovibrational eigenstates must be checked $a$ posteriori whether they exist or not.

As a straightforward example, let us consider a triatomic molecule where two identical spin-1/2 nuclei (fermions) can be exchanged. In the case of the three $\mathrm{H}_{2}{ }^{n} \mathrm{O}, n=16,17$, and 18, isotopologues of water, there are two separate rovibrational energy level sets corresponding to the two distinct molecules. The two sets are traditionally called ortho and para, with the convention that the ortho form has the higher spin-statistical weight. In the case of water, these distinct principal components of the spectroscopic network (SN) (Császár and Furtenbacher, 2011, Furtenbacher and Császár, 2012) of the $\mathrm{H}_{2}{ }^{n} \mathrm{O}$ molecules are not connected by measured transitions. Note, however, that due to the allowed coupling of nuclear spin with rovibronic motions, nuclear spin symmetry-breaking mixings and ortho to para transitions have been observed (Ozier et al., 1970, Bordé et al., 1980, Pique et al., 1984, Quack, 2011).

\subsection{Wavefunction analysis tools via projection techniques}

Besides the exact $J$ and $\Gamma_{\alpha}$ labels introduced in the previous sections it is useful to assign approximate quantum numbers to the computed rovibrational eigenstates, such as the zerothorder HO and RR quantum numbers, as these labels are widely used in experimental highresolution spectroscopy and they also help capture the dynamics embodied in the eigenstates. This task can be completed by decomposing the numerically exact variational eigenstates in the basis of appropriately chosen zeroth-order states.

The normal mode decomposition (NMD) technique (Mátyus et al., 2010) has been developed to facilitate the assignment of zeroth-order $\mathrm{HO}$ quantum numbers to variational vibrational eigenstates $\Phi_{i}$ by calculating the

$$
\mathrm{NMD}_{i j}=\left|\left\langle\Phi_{i} \mid \Phi_{j}^{\mathrm{HO}}\right\rangle\right|^{2}
$$

coefficients, where $\Phi_{j}^{\mathrm{HO}}$ denotes a $\mathrm{HO}$ eigenstate. Labelling $\Phi_{i}$ with $\mathrm{HO}$ quantum numbers can be accomplished by finding the dominant $\mathrm{NMD}_{i a}$ coefficient (if it exists) and assign $\Phi_{i}$ with the HO quantum numbers of $\Phi_{a}^{\mathrm{HO}}$.

The rigid rotor decomposition (RRD) method (Mátyus et al., 2010, Szidarovszky et al., 2012) is a useful tool for the assignment of variationally-computed rovibrational energy levels and eigenstates. The initial step is the evaluation of the

$$
\operatorname{RRD}_{i, j k}=\left|\left\langle\Psi_{i} \mid \Phi_{j} R_{k}\right\rangle\right|^{2}
$$

coefficients, where $\Psi_{i}$ is the rovibrational eigenstate to be assigned with approximate vibrational and rotational quantum numbers, while $\Phi_{j}$ and $R_{k}$ denote variational vibrational and rigid-rotor eigenstates, respectively. After finding the dominant $\Phi_{a} R_{b}$ contribution in $\Psi_{i}$, it is straightforward to assign $\Psi_{i}$ with the vibrational labels of $\Phi_{a}$ and the rigid-rotor quantum numbers [( $\left.J, K_{\mathrm{a}}, K_{\mathrm{c}}\right)$ for asymmetric tops, and $(J, K)$ for symmetric tops] of $R_{b}$. 
Projection techniques are also useful to attach rovibrational labels to the intermolecular motions of clusters, in particular dimers, highlighting their dynamical behavior. These techniques can be employed both for bound and resonance states, as demonstrated for the $\mathrm{CH}_{4} \cdot \mathrm{H}_{2} \mathrm{O}$ dimer (Sarka et al., 2016, 2017) and the $\mathrm{HeH}_{2}^{+}$complex (Papp et al., 2018), respectively.

The so-called coupled-rotor decomposition (CRD) technique (Sarka et al., 2017) is relevant to weakly-bound dimers, where the intermonomer bonding is very weak compared to the bonding within the monomer units, denoted as $A$ and $B$. In these dimers, an example is $\mathrm{CH}_{4} \cdot \mathrm{H}_{2} \mathrm{O}$, considering the two monomer units rigid is an excellent model. Then the description of the lowenergy vibrational motions of the dimer requires only six dofs. To obtain a description of the $6 \mathrm{D}$ eigenstates of the dimer based on the picture of coupled monomers with hindered rotation, one needs to do the following: (a) Fix the $A-B$ separation at a given value. (b) Set the PES to zero and compute the rovibrational states in this $5 \mathrm{D}$ model using exactly the same coordinate and grid representation as employed for the 6D model. (c) Calculate the eigenenergies of the CR model, characterized by the $J$ total angular momentum quantum number, with respect to the energies of states of the rigid monomers characterized by $j_{A}$ and $j_{B}$ :

$$
E^{\mathrm{CR}}\left(j_{A}, j_{B}, j, J\right)=E_{A}^{\mathrm{RR}}\left(j_{A}\right)+E_{B}^{\mathrm{RR}}\left(j_{B}\right)+E_{c}(j, J),
$$

where $j$ is the internal angular momentum quantum number of the $A B$ diatomic, responsible for coupling the angular momenta of $A$ and $B$, and $E_{c}(j, J)$ is the related coupling energy, whose computation is detailed in Brocks et al. (1983). (d) At "infinite" separation, $E^{\mathrm{CR}}\left(j_{A}, j_{B}, j, J\right)$ is simply the sum of the rigid-rotor energies of the monomers, allowing straightforward labeling of the CR states. (e) In a DVR representation it is also straightforward to compute the overlaps of the 5D and 6D eigenstates (Sarka et al., 2017); computation and analysis of the CRD overlap coefficients completes the CRD assignment of the $6 \mathrm{D}$ eigenstates. A scheme very similar to the CRD one can be employed for the characterization of computed rovibrational resonance states, as described by Papp et al. (2018).

Note in the end that sometimes it seems more effective to base the assignment of dynamical labels to the computed eigenstates not on wavefunctions but on one- and two-mode reduced density matrices (Šmydke and Császár, 2019). Overlaps computed between densities of states already assigned and the list of new, assignable states seemingly provides an efficient route toward developing a large set of semi-automatically assigned states.

\section{Computation of rovibrational resonances}

Rovibrational resonances, also known as metastable or quasibound rovibrational states, are not mere curiosities of nuclear-motion theory arising within the BO approximation but they are at the heart of chemistry, especially when bimolecular chemical reactions, including reactive collisions, are considered. Rovibrational resonances are also met in predissociation and photodissociation studies as well as in unimolecular decays of polyatomics. The efficient computation and characterization of rovibrational resonances forms an essential and extremely important part of modern quantum chemistry.

While bound rovibrational states are assumed to have infinite lifetimes, resonances are usually 
characterized by two parameters: resonance positions and resonance lifetimes. These parameters can be computed within time-dependent and time-independent frameworks (Moiseyev, 2011, Klaiman and Gilary, 2012).

Hereby we restrict ourselves to time-independent approaches, in which resonances are identified as eigenstates of the rovibrational Hamiltonian having complex eigenvalues. These eigenvalues are usually written, in atomic units, as

$$
E_{n}^{\mathrm{res}}=\epsilon_{n}-\frac{\mathrm{i}}{2} \Gamma_{n}
$$

where $\epsilon_{n}=\operatorname{Re}\left(E_{n}^{\text {res }}\right)$ is the resonance position, while $\Gamma_{n}$ is the resonance width, related to the inverse lifetime,

$$
\rho(\mathrm{Q}, t)=\left|\Psi_{n}^{\mathrm{res}}\right|^{2} \propto e^{-\Gamma_{n} t},
$$

where Q represents a point in coordinate space. Due to the complex nature of the eigenvalues, the corresponding $\Psi_{n}^{\text {res }}$ wavefunctions are not normalizable, i.e., they diverge exponentially as the dissociation coordinate goes to infinity. This makes it necessary to apply special computational tools, some of which are detailed below, for the determination of resonance eigenstates. Similar to bound states, analysis of resonance wavefunctions gives insight into the physical properties of the metastable eigenstates, for example into the mechanisms responsible for their dissociation (or other dynamical behavior).

We briefly review in this section three techniques for computing rovibrational resonances: the stabilization method (Hazi and Taylor, 1970, Lefebvre, 1985, Riera, 1993, Mandelshtam et al., 1993, Moiseyev, 2011), the technique of complex coordinate scaling (CCS) (Moiseyev et al., 1981, Moiseyev, 2011), and the use of complex absorbing potentials (CAP) (Vibók and BalintKurti, 1992, Riss and Meyer, 1993, Skokov et al., 1999, Halász and Vibók, 2000, Mussa and Tennyson, 2002, Poirier and Carrington Jr., 2003a,b, Muga et al., 2004).

\subsection{The stabilization method}

The simplest approach to compute long-lived rovibrational resonances is offered by the socalled stabilization method (Hazi and Taylor, 1970, Lefebvre, 1985, Riera, 1993, Mandelshtam et al., 1993, Moiseyev, 2011). The attractiveness of the stabilization method lies in its simplicity, since it allows the use of bound-state variational nuclear-motion codes for computations yielding rovibrational resonance positions and even resonance widths (Simons, 1981, Macías and Riera, 1984, 1989).

In the stabilization method the eigenvalues above the first dissociation asymptote are monitored, while several (on the order of 10-20) standard, Hermitian, variational nuclear-motion computations are carried out with slightly different computational parameters chosen along the coordinate describing dissociation. These parameters can be a scaling factor in a basis (Macías and Riera, 1984), the size of the box containing the system (Lefebvre, 1985), and/or the number of DVR basis functions. The "continuum" energies obtained vary significantly with changes in the bases. Resonance energies are obtained by identifying eigenvalues which are converged well and are insensitive to the (small) changes in the dissociative parameters. 
The results obtained from the stabilization method can most easily be visualized in the form of a histogram. In the stabilization histogram, the eigenvalues computed at different ranges of the dissociation coordinate that fall in the respective bins are counted. Resonance energies, which are located above the dissociation threshold and are converged tightly appear in the histogram as clear peaks (Hazi and Taylor, 1970, Mandelshtam et al., 1993, Papp et al., 2017a). Resonance widths can also be obtained using the stabilization method; see, for example, citet81Simons, Macías and Riera (1989), Mandelshtam et al. (1994), and Haritan and Moiseyev (2017).

\subsection{The technique of complex coordinate scaling (CCS)}

An alternative approach for computing resonance eigenstates using bound-state nuclear-motion algorithms is to transform the time-independent nuclear Schrödinger equation, $\hat{H} \Psi^{\mathrm{res}}=E^{\mathrm{res}} \Psi^{\mathrm{res}}$, to

$$
\hat{S} \hat{H} \hat{S}^{-1} \hat{S} \Psi^{\mathrm{res}}=E^{\mathrm{res}} \hat{S} \Psi^{\mathrm{res}}=\left(\hat{S} \hat{H} \hat{S}^{-1}\right) \Phi=E^{\mathrm{res}} \Phi,
$$

such that $\Phi=\hat{S} \Psi^{\text {res }}$ becomes square integrable and can be expanded using standard bound-state basis sets. The probably best known transformation is the so-called complex coordinate scaling (CCS) (Moiseyev et al., 1981, Moiseyev, 2011). The simplest implementation of CCS involves a parameter-dependent operator $\hat{S}(\theta)$, which rotates the dissociation coordinate $R$ in the complex plane by the angle $\theta, \hat{S}(\theta) f(R)=f\left(R e^{i \theta}\right)$ (Moiseyev, 2011). In practice, the eigenvalues of the non-Hermitian $\hat{S}(\theta) \hat{H} \hat{S}^{-1}(\theta)$ operator are computed for many different values of $\theta$, forming eigenvalue trajectories on the complex plane, and resonance eigenvalues are identified as cusps or similar features (Moiseyev et al., 1981) in the trajectories.

The authors' implementation of the CCS method, called D²FOPI-CCS (Szidarovszky et al., 2010, Szidarovszky and Császár, 2013), was obtained by extending the tailor-made variational nuclear-motion code $D^{2}$ FOPI (Szidarovszky et al., 2010) with the conventional CCS as well as the standard exterior CCS methods, detailed to some extent in Szidarovszky and Császár (2013) and Papp et al. (2018), respectively. In the $\mathrm{D}^{2} \mathrm{FOPI}-\mathrm{CCS}$ protocol all the bound rovibrational eigenstates and hundreds of eigenpairs above the first dissociation threshold are computed as a first step, then these eigenstates are used as basis functions to construct the matrix representation of the complex-coordinate-scaled rovibrational Hamiltonian.

\subsection{Complex absorbing potentials (CAP)}

A third possibility to compute rovibrational resonances is offered by the technique of complex absorbing potentials (CAP) (Riss and Meyer, 1993, Muga et al., 2004, Mussa and Tennyson, 2002, Skokov et al., 1999), which involves perturbing the rovibrational Hamiltonian by a CAP function that is non-zero only in the asymptotic region of the dissociative coordinate. The CAP is chosen such that the perturbed non-Hermitian Hamiltonian has square-integrable wavefunctions with corresponding complex eigenvalues that are good approximations to the resonance eigenvalues. In practice, the CAP function is associated with a strength parameter $\eta$, so that the perturbation of the Hamiltonian can be written as

$$
\hat{H} \rightarrow \hat{H}(\eta)=\hat{H}-\mathrm{i} \eta \hat{W}
$$


where $\hat{W}$ is the CAP function, its argument(s) are the dissociation coordinate(s). $\hat{W}$ is typically chosen to be non-zero only in the asymptotic regions of the PES, and the optimal functional form of $\hat{W}$ has been the topic of many studies, see, for example, Vibók and Balint-Kurti (1992), Halász and Vibók (2000), Poirier and Carrington Jr. (2003a), and Poirier and Carrington Jr. (2003b). The eigenvalues of the non-Hermitian operator $\hat{H}(\eta)$ are computed for many different values of $\eta$, forming eigenvalue trajectories on the complex plane, and resonance eigenvalues are identified as cusps in the trajectories.

The authors' implementation of the CAP method, called GENIUSH-CAP (Papp et al., 2017b, Simkó et al., 2019), is an extension of the GENIUSH code (Mátyus et al., 2009, Fábri et al., 2011a) in which a CAP is added to the standard rovibrational Hamiltonian, and the matrix representation of the CAP-perturbed Hamiltonian is constructed in the basis of the eigenvectors of the unperturbed Hamiltonian. The GENIUSH-CAP code inherits all the advantages of the GENIUSH code; thus, it allows full- and reduced-dimensional computations of rovibrational resonances in a black-box-type fashion. Efficient algorithms moving the computation of rovibrational resonances toward a black-box-type technique have appeared (Tremblay and Carrington, 2005, Simkó et al., 2019).

\subsection{Wavefunction analysis tools}

One of the advantages of the GENIUSH-CAP code (Papp et al., 2017b, Simkó et al., 2019) is that beyond full- and reduced-dimensional resonance computations, reduced-dimensional model computations can be carried out with the GENIUSH code (Mátyus et al., 2009, Fábri et al., 2011a) for the system whose resonances are investigated. This allows for a straightforward computation of overlaps between the resonance wave functions determined by GENIUSHCAP and model wavefunctions computed by GENIUSH. Based on such overlaps, quantum numbers, dissociation branching ratios, etc. can be determined in a rigorous and efficient way, see, for example, Papp et al. (2017a) and Papp et al. (2018). This type of overlap-based analysis is very similar in spirit to the RRD (Mátyus et al., 2010) and the CRD (Sarka et al., 2017) schemes described above.

\section{Applications}

Up to now we have focused on the most important technical details characterizing some of the fourth-age quantum-chemical nuclear-motion protocols, allowing the efficient variational computation of rovibrational bound states and resonances. Next, we present a couple of representative results of computations based on these protocols, obtained mostly in our laboratory. The results, i.e., energy levels, eigenstates, assignments, and time-dependent quantum-dynamical results, have applications in many different areas of science and engineering, which, in order to save space, we are going to cover only occasionally.

The computations whose results are reported in this section utilized the following in-house codes: D²FOPI (Czakó et al., 2004, Furtenbacher et al., 2006, Szidarovszky et al., 2010), D²FOPI-CCS (Szidarovszky et al., 2010, Szidarovszky and Császár, 2013), GENIUSH (Má- 
tyus et al., 2009, Fábri et al., 2011a), and GENIUSH-CAP (Papp et al., 2017b). D²FOPI is a tailor-made DVR-based code designed for computing bound rovibrational states of triatomic molecules. $\mathrm{D}^{2} \mathrm{FOPI}-\mathrm{CCS}$ is an extension of $\mathrm{D}^{2} \mathrm{FOPI}$, utilizing the CCS (Moiseyev, 2011) method, and allows the computation of rovibrational resonance states. In $D^{2}$ FOPI-CCS the CCS Hamiltonian is represented in the eigenstate basis of the untransformed rovibrational Hamiltonian. GENIUSH, which stands for a general (GE) rovibrational code with a numerical $(\mathrm{N})$, internal-coordinate (I), user-specified (US) Hamiltonian $(\mathrm{H})$, computes rovibrational bound states by numerically representing not only the potential (through a DVR) but also the kinetic energy operator of a molecular system. Any number of reduced-dimensional models of vibrating-rotating molecules can straightforwardly be defined within GENIUSH. GENIUSHCAP is an extension of GENIUSH, in which a CAP (Riss and Meyer, 1993, Muga et al., 2004, Mussa and Tennyson, 2002, Skokov et al., 1999) is added to the standard rovibrational Hamiltonian, allowing for the computation of resonance states. In GENIUSH-CAP the matrix representation of the CAP-perturbed Hamiltonian is constructed using the basis of the eigenvectors of the unperturbed Hamiltonian. In all the codes mentioned different versions of iterative Lanczos eigensolvers are used to determine the desired eigenvalues and eigenvectors.

\subsection{Computation of all the bound (ro)vibrational eigenstates}

There are only a few strongly-bound polyatomic molecules for which all the bound (ro)vibrational eigenstates have been computed. This is not surprising when one considers the fact that even for a triatomic molecule this may involve the computation of a couple of million eigenstates (Simkó et al., 2017). The formidable task of computing these eigenstates is helped tremendously by the fact that the quantum number corresponding to overall rotation, $J$, is one of the good quantum numbers in a field-free case; thus, the Hamiltonian $\mathbf{H}$ is block-diagonal in $J$, with block sizes proportional to $2 J+1$. Due to dissociation limit(s) the largest block is at a $J$ value considerably smaller than the $J_{\max }$ value characterizing the highest bound rovibrational state.

\subsection{1 $\mathrm{H}_{2}{ }^{16} \mathrm{O}$ and its isotopologues}

Several BO and adiabatic PESs are available for water and its isotopologues (Partridge and Schwenke, 1997, Polyansky et al., 2003, Barletta et al., 2006, Shirin et al., 2008, Polyansky et al., 2018), most facilitate the determination of all bound states of these molecules (Maksyutenko et al., 2007). The first-principles (ab initio) PESs of water, especially after including several small correction terms [scalar relativistic (Császár et al., 1998b, Quiney et al., 2001), diagonal Born-Oppenheimer (Zobov et al., 1996), and quantum electrodynamics (QED) (Pyykkö et al., 2001) corrections], all become fairly accurate. In fact, water is the first polyatomic, polyelectronic molecule for which a purely $a b$ initio PES could provide sub-cm ${ }^{-1}$ accuracy in a variational computation of all measured transitions (Polyansky et al., 2003). When ab initio PESs are adjusted to the large number of "measured" rovibrational energy levels (Tennyson et al., 2013), the accuracy of the thus derived semiempirical PESs increases by somewhat more than an order of magnitude. Nevertheless, the accuracy of rovibrational states computed with the best semiempirical PES and an exact KEO is still orders of magnitude lower than those of high-resolution spectroscopic measurements (Tennyson et al., 2009, 2010, 2013, 2014a,b). 
$\mathrm{H}_{2}{ }^{16} \mathrm{O}$ has about 1150 vibrational $(J=0)$ states (Császár et al., 2010). The corresponding numerical results, the wavefunction plots (Császár et al., 2010), and the assignments reveal interesting characteristics about the energy-level structure and the dynamics of the $\mathrm{H}_{2}{ }^{16} \mathrm{O}$ molecule. The plots and the assignments show, for example, how the convenient normal-mode picture of vibrations characterizing states of $\mathrm{H}_{2}{ }^{16} \mathrm{O}$ with low excitation changes to a local-mode picture, the effect the low barrier to linearity (Császár et al., 1998a, Valeev et al., 2001) has on the characteristics of the states, and the onset of quantum monodromy (Zobov et al., 2005).

As water is the most prevalent polyatomic molecule in the universe, generation of a detailed line list of the rovibrational transitions is of utmost importance for many scientific and engineering applications. Thus, it is not surprising that several line lists have been developed, the most important ones are probably Partridge and Schwenke (1997), Barber et al. (2006), Gordon et al. (2017), and Polyansky et al. (2018). Note that while Partridge and Schwenke (1997) and Barber et al. (2006) contain 300 and 500 million transitions, respectively, Polyansky et al. (2018) lists 5 billion rovibrational transitions. These transitions have been computed variationally based on a highly accurate semiexperimental PES (Furtenbacher et al., 2016, Polyansky et al., 2018).

If all the bound rovibrational energies are available from detailed nuclear-motion computations, they can be used to determine ideal-gas partition functions of molecules (Mayer and Mayer, 1940, Herzberg, 1945, Lewis et al., 1961, McQuarrie, 2000, Furtenbacher et al., 2016, Simkó et al., 2017). For this purpose, the total partition function is assumed to be the product of the internal and the translational partition functions. The latter can be computed exactly, only the internal partition function will be approximate, even for an ideal gas. As also well known, the internal partition function, $Q_{\text {int }}$, of a free molecule can be calculated as

$$
Q_{\text {int }}=g_{\mathrm{s}} \sum_{i} g_{i}\left(2 J_{i}+1\right) \exp \left(\frac{-c_{2} E_{i}}{T}\right)
$$

called direct summation, where $c_{2}=h c / k_{\mathrm{B}}$ is the second radiation constant, $J_{i}$ is the rotational quantum number, $E_{i}$ is the rovibrational energy level given in $\mathrm{cm}^{-1}$ (the zero is taken as the lowest existing (ro)vibrational state), $T$ is the thermodynamic temperature in $\mathrm{K}, g_{\mathrm{s}}$ is a stateindependent nuclear-spin degeneracy factor for atoms not exchanged under rotation (Herzberg, 1945), $g_{i}$ is the nuclear-spin degeneracy factor for identical atoms interchanged under rotation, and the index $i$ runs over all possible rovibronic energies considered. The availability of about 20000 "measured" (better say "empirical") rovibrational energy levels (with an almost complete coverage up to $9200 \mathrm{~cm}^{-1}$ ) for $\mathrm{H}_{2}{ }^{16} \mathrm{O}$ (Tennyson et al., 2013), augmented with all the computed bound energy levels, means that $Q_{\text {int }}$ of $\mathrm{H}_{2}{ }^{16} \mathrm{O}$ (Furtenbacher et al., 2016), as well as $Q_{\text {int }}$ s of $\mathrm{D}_{2}{ }^{16} \mathrm{O}$ (Simkó et al., 2017), $\mathrm{D}_{2}{ }^{17} \mathrm{O}$ (Simkó et al., 2017), and $\mathrm{D}_{2}{ }^{18} \mathrm{O}$ (Simkó et al., 2017), and thus that of heavy water (Simkó et al., 2017), have the ultimate accuracy at lower temperatures and an accuracy better than $1 \%$ even at $6000 \mathrm{~K}$. Note that due to their lower ZPVE values and lower fundamentals, the $\mathrm{D}_{2} \mathrm{O}$ species have almost three million bound rovibrational states (Simkó et al., 2017), while $\mathrm{H}_{2}{ }^{16} \mathrm{O}$ has only less than one million.

\subsection{2 $\mathrm{H}_{3}^{+}$and its deuterated isotopologues}

$\mathrm{H}_{3}^{+}$is an extremely important molecule in interstellar space as it governs basically the whole gas-phase chemistry of the interstellar medium (ISM) (Herbst, 2000). 
In 2006, based on a PES (Munro et al., 2006) with correct asymptotic behavior, Tennyson et al. (Tennyson et al., 2006, Munro et al., 2006) computed almost all of the vibrational energy levels corresponding to the electronic ground state of $\mathrm{H}_{3}^{+}$. Szidarovszky et al. (2010), using the same PES but a different protocol, confirmed the results of the earlier study and determined 1287 (counting the $E$-symmetry states twice) bound vibrational states. It is believed that a convergence of at least $1 \mathrm{~cm}^{-1}$ was achieved in even for states very close to the first dissociation asymptote, placed at $D_{0}=34911.6 \mathrm{~cm}^{-1}$.

$\mathrm{H}_{3}^{+}$is the polyatomic molecule for which the most accurate ab initio adiabatic PES has been computed (Pavanello et al., 2012b,a, Polyansky et al., 2012). To achieve this accuracy required the use of special techniques of electronic structure theory and the consideration of (scalar) relativistic (Cowan and Griffin, 1976, Tarczay et al., 2001), quantum electrodynamics (Pyykkö et al., 2001), and diagonal Born-Oppenheimer (Handy et al., 1986) corrections obtained based on highly-accurate electronic wavefunctions. The accuracy achieved allowed the straightforward assignment of near-IR measured spectra (Pavanello et al., 2012b). The accuracy of the adiabatic PES is in fact so high that comparison of the computed and measured rovibrational energies clearly revealed the role of nonadiabatic effects on the computed levels (Furtenbacher et al., 2013b). Modeling of nonadiabatic effects is one of the last frontiers of computational molecular spectroscopy and it is likely that the advances will employ $\mathrm{H}_{3}^{+}$as the test molecule. These tests will likely utilize the empirical rovibrational energy level sets of $\mathrm{H}_{3}^{+}$(Furtenbacher et al., 2013b), $\mathrm{H}_{2} \mathrm{D}^{+}$(Furtenbacher et al., 2013a), and $\mathrm{HD}_{2}^{+}$(Furtenbacher et al., 2013a).

One of the great remaining challenges of $\mathrm{H}_{3}^{+}$spectroscopy is the understanding of the CarringtonKennedy experiments (Carrington et al., 1982, Carrington and Kennedy, 1984), revealing an extraordinary number of rovibrational transitions around the first dissociation asymptote. This requires an extremely accurate PES, new methodological developments for the computation of bound and resonance states, and even perhaps new experiments at low temperatures producing much less cluttered spectra.

\subsection{Rovibrational computations on quasistructural molecules}

\subsection{1 $\mathbf{H}_{5}^{+}$}

The $\mathrm{H}_{5}^{+}$molecular ion lacks the usual (heavier) central atom(s) that may form multiple strong (covalent, often 2c-2e) bonds, typical for virtually all small molecular species. Thus, unlike in the case of semirigid molecules (like $\mathrm{H}_{2} \mathrm{O}$ and $\mathrm{H}_{3}^{+}$), the lack of a well-defined (effective) structure makes the low- and especially the high-resolution spectra of $\mathrm{H}_{5}^{+}$particularly challenging to anticipate and interpret (Fábri et al., 2014c, Sarka et al., 2015, Sarka and Császár, 2016). It has also become clear that it is not trivial to set up meaningful low-dimensional model Hamiltonians to describe the complex internal dynamics of this ion, governed by the following three large-amplitude motions: hopping of the central proton $(\mathrm{PH})$, torsion $(\mathrm{T})$, and scrambling of the hydrogens of the formal $\mathrm{H}_{3}^{+}$subunit (SC). Nevertheless, even for this cation (formally $\mathrm{H}_{3}^{+}$ solvated by a $\mathrm{H}_{2}$ molecule at one of the vertices of $\mathrm{H}_{3}^{+}$) (a) free motion (no hindering potential) works well for describing the 1D torsional motion of the two $\mathrm{H}_{2}$ moieties in the sides of the central proton; (b) coupling the torsional motion with one of the rotational dofs provides 
a meaningful model to interpret the structure of the lowest rovibrational energy levels; and (c) the unfeasible scrambling motion, hindered by a relatively substantial barrier, lowers the high permutational symmetry of the system.

The very unusual nuclear dynamics of $\mathrm{H}_{5}^{+}$holds true for all D-substituted isotopomers and isotopologues, $\mathrm{H}_{5-n} \mathrm{D}_{n}, n=0-5$ (Sarka and Császár, 2016). The computations strongly suggest that the unusual dynamical behavior is connected to the shape of the PES and not to the fivefold permutation symmetry of the molecule. Briefly, the detailed nuclear-motion computations (Fábri et al., 2014c, Sarka et al., 2015, Sarka and Császár, 2016) suggest that (a) the six possible D-substituted isotopologues form 12 isotopomers; (b) to interpret the rovibrational energy levels, one needs to explicitly consider that the effective value of the $A$ rotational constant is twice as large as its equilibrium value and the nearly-free torsion is strongly coupled to the rotational dof corresponding to the torsional axis; (c) though some isotopomers, namely [DH$\mathrm{H}-\mathrm{DH}]^{+}$and $[\mathrm{DH}-\mathrm{D}-\mathrm{DH}]^{+}$, show more chaotic behavior than others, labeling of a number of states involving coordinates corresponding to the T, PH, and $\mathrm{SC}$ motions is possible, yielding some understanding of the quantum dynamics of these ions; (d) the splittings showed by certain torsion-rotation energy level pairs can be attributed to the pertubation of free internal rotation by the weak torsional potential instead of tunneling between the two equivalent torsional potential wells; and (e) the hopping mode is sensitive to the identity of the middle atom but more or less independent of the sides. These characteristics of the internal dynamics make $\mathrm{H}_{5}^{+}$and its deuterated isotopologues members of the family of quasistructural molecules (Császár et al., 2019).

\subsection{2 $\mathbf{C H}_{5}^{+}$}

Based on a considerable number of experimental (White et al., 1999, Asvany et al., 2005, Ivanov et al., 2010, Oka, 2015, Asvany et al., 2015, Brackertz et al., 2017), computational (Schreiner et al., 1993, Padma Kumar and Marx, 2006, Huang et al., 2006, Wang and Carrington Jr., 2008, Wodraszka and Manthe, 2015, Wang and Carrington Jr., 2016, Fábri et al., 2017), and modeling (Bunker, 1996, Kolbuszewski and Bunker, 1996, East and Bunker, 1997, East et al., 1997, Bunker et al., 2004, Schmiedt et al., 2016, 2017a,b, Fábri and Császár, 2018) studies, it is transparent that the rovibrational energy level pattern of the protonated methane molecule, $\mathrm{CH}_{5}^{+}$, defies any description attempts based on the RRHO model. The computations reported in Fábri et al. (2017), Wang and Carrington Jr. (2016), and Wang and Carrington Jr. (2008) yielded vibrational and rovibrational energy levels for $\mathrm{CH}_{5}^{+}$for 7D bend and 12D models. These results prove numerically that the commonly applied separation of rotations and vibrations completely breaks down for $\mathrm{CH}_{5}^{+}$, as predicted by group-theoretical arguments (Wodraszka and Manthe, 2015, Schmiedt et al., 2015).

Given the extraordinary difficulties related to the dynamics of $\mathrm{CH}_{5}^{+}$, it is of considerable interest to note that a simple and intuitive model, the vibrational quantum-graph model (Fábri and Császár, 2018), is able to provide a qualitative explanation for the unusual low-energy vibrational quantum dynamics of $\mathrm{CH}_{5}^{+}$. The vertices of the quantum graph represent versions of the equilibrium structure with distinct atom numbering (120 possibilities for $\mathrm{CH}_{5}^{+}$), while the edges refer to collective nuclear motions (torsional and flip motions for $\mathrm{CH}_{5}^{+}$) transforming the versions of the equilibrium structure into one another. Note that it was (Nefedova et al., 1993) 
who introduced to chemistry the graphical representation "of the symmetries of [the] PES of nonrigid molecule[s], whose nuclear motion wavefunctions are delocalized over several equalenergy global minima" (the molecules considered were $\mathrm{ArH}_{3}^{+}, \mathrm{C}_{2} \mathrm{H}_{3}^{+}, \mathrm{CH}_{4}^{+}$, and $\mathrm{LiBH}_{4}^{+}$). The novel vibrational quantum-graph model allows the mapping of the complex vibrational quantum dynamics of $\mathrm{CH}_{5}^{+}$onto the motion of a particle confined in a quantum graph. The vibrational energy levels are obtained by solving the one-dimensional time-independent nuclear Schrödinger equation, subject to appropriate boundary conditions. Furthermore, due to the low barriers hindering the torsional and flip motions in $\mathrm{CH}_{5}^{+}$, the motion along the edges of the quantum graph can safely be assumed to be free. The quantum-graph model is able to reproduce the lowestlying vibrational energy levels of $\mathrm{CH}_{5}^{+}$(and $\mathrm{CD}_{5}^{+}$) with remarkable accuracy (Fábri and Császár, 2018). Note that a five-dimensional rigid-rotor model, based on the combination of the three rotational dofs with the two soft vibrational modes (torsion and flip), is also able to explain the unusual rovibrational energy structure of $\mathrm{CH}_{5}^{+}$(Schmiedt et al., 2016, 2017a,b).

In summary, while we are close to understanding the highly unusual nuclear dynamics, and even perhaps the related spectra, of $\mathrm{CH}_{5}^{+}$via variational nuclear-motion computations, it is safe to say that this cation remains the premier representative of quasistructural molecules (Császár et al., 2019), providing a very challenging playground for both experiment and theory.

\subsection{Computation of rovibrational resonances}

\subsection{1 $\quad \mathrm{H}_{2} \mathrm{O}$}

Szidarovszky and Császár (2013) computed the low-lying rovibrational resonances of the stronglybound $\mathrm{H}_{2} \mathrm{O}$ molecule using the CCS method. The calculations aided the proper assignment of some observed rovibrational transitions beyond the first dissociation limit of $\mathrm{H}_{2}{ }^{16} \mathrm{O}$ (Zobov et al., 2011). Furthermore, by inspecting the vibrational probability density plots from the stationary resonance calculations, several types of (dynamical) dissociation behavior, varying among the states, could be identified.

\subsubsection{Ar $\cdot \mathrm{NO}^{+}$}

Vibrational resonances of the vdW complex Ar$\cdot \mathrm{NO}^{+}$were studied in Papp et al. (2017a) using the CAP technique and the stabilization method. It was found that the results obtained are in good agreement with those derived from close-coupling scattering computations (Stoecklin et al., 2002), and that the close-coupling scattering and rovibrational resonance approaches complement each other remarkably well. Comparison with the limited experimental information confirmed the experimental results obtained, and shed light to more subtle dynamics than initially predicted based on the observations.

A particularly interesting feature of the computed rovibrational resonances of $\mathrm{Ar} \cdot \mathrm{NO}^{+}$is the repetition of the basically same set of levels for each vibrational excitation of the $\mathrm{NO}^{+}$stretch. The dissociation energy of Ar$\cdot \mathrm{NO}^{+}$is about $800 \mathrm{~cm}^{-1}$ but due to the almost perfect adiabatic separation of the $\mathrm{NO}^{+}$stretch from the other two vibrational dofs, basically the same energy- 
level structure is computed even at about $8000 \mathrm{~cm}^{-1}$.

\subsection{3 $\quad \mathrm{H}_{2} \mathrm{He}^{+}$}

Using the CCS, the CAP, and the stabilization methods, a large number of rovibrational resonances have been computed and characterized in Papp et al. (2018) for $\mathrm{H}_{2} \mathrm{He}^{+}$. It is hoped that these accurate computations facilitate the first experimental observation of rovibrational transitions of this fundamental molecule, made up of the two most abundant elements of the universe. Note that $\mathrm{HHe}^{+}$has just been observed in the interstellar medium (Güsten et al., 2019).

Alongside the spectroscopic data valuable for future high-resolution experiments, the stabilization mechanism of the long-lived resonances, possible dissociation pathways, and dissociation branching ratios could also be determined from the quantum-chemical computations. These results provide insight into the complex physics and the rich dynamics characterizing this system. Because the $\mathrm{H}-\mathrm{H}$ stretching fundamental lies above the first dissociation threshold of $D_{0}=1775.4 \mathrm{~cm}^{-1}$, resonances are expected to play a crucial role in the collision and association reaction involving $\mathrm{H}_{2} \mathrm{He}^{+}$, including radiative association and radiative charge-transfer reactions.

\subsection{4 $\quad \mathrm{H}_{2} \cdot \mathrm{CO}$}

Papp et al. (2017b) computed the vibrational resonances of the weakly-bound complex $\mathrm{H}_{2} \cdot \mathrm{CO}$ using the GENIUSH-CAP approach and a four-dimensional model PES. This was one of the first examples where vibrational resonances could be determined for a system containing more than three atoms.

The computations allowed to identify and characterize resonances of both para- and ortho$\mathrm{H}_{2} \cdot \mathrm{CO}$. Quantum number assignments for the resonances were achieved by inspecting the vibrational probability density plots and by computing wavefunction overlaps with eigenstates of reduced-dimensional models, as discussed briefly in Section 4.4.

\subsection{Stationary-state computations serving dynamical studies}

In this penultimate section we mention briefly a few enlightening examples where variational stationary-state computations have been utilized to obtain detailed information on certain dynamical processes of molecular systems.

Szidarovszky and Yamanouchi (2017) simulated the laser-induced alignment dynamics of the weakly-bound $\mathrm{H}_{2} \mathrm{He}^{+}$complex to investigate the effects of non-rigidity and rovibrational couplings on the rotational dynamics of the system. For this, variationally-computed rovibrational states of the $\mathrm{H}_{2} \mathrm{He}^{+}$complex were used as basis functions to expand the laser-induced rovibrational wave packet. It was shown that centrifugal distortion and rovibrational couplings indeed influence strongly the laser-induced rotational dynamics of the system; thus, the standard protocol of applying the RR approximation for simulating such processes needs to be used with 
considerable care.

In the case of $\mathrm{H}_{2} \mathrm{O}$, a molecule much more rigid than $\mathrm{H}_{2} \mathrm{He}^{+}$, deviations from the RR approximation in its laser-induced alignment dynamics can also arise, mostly when one-photon transitions inducing vibrational excitations occur (Szidarovszky and Yamanouchi, 2018). In this case the origin of the deviation is due less to centrifugal distortion, it stems mostly from the inadequacy of the RR model to describe optical selection rules of rotational transitions associated with vibrational excitation.

In the experiments described in Larimian et al. (2016), the $\mathrm{C}_{2} \mathrm{H}_{2}^{2+}$ and $\mathrm{C}_{2} \mathrm{H}_{4}^{2+}$ dications were generated by doubly ionizing their neutral parents using few-cycle intense laser pulses. For both $\mathrm{C}_{2} \mathrm{H}_{2}^{2+}$ and $\mathrm{C}_{2} \mathrm{H}_{4}^{2+}$, in the deprotonation pathway of their Coulomb explosion, an ultraslow, microsecond timescale exponential decay channel was observed. Reduced-dimensional resonance-state computations revealed that in both cases the slow decay channel is due to quasibound states along the $\mathrm{C}-\mathrm{H}$ vibrational mode, where tunneling through a barrier is responsible for the exponential decay.

Due to the rapidly increasing amount of high-quality data placed into molecular spectroscopy databases, it is worth mentioning here that laser-induced dynamics can also be simulated for molecules for which spectroscopic databases, containing rovibrational energy levels as well as transition amplitudes between the corresponding eigenstates, are available. This interesting approach was pursued, for example, in Owens et al. (2017) and Schuh et al. (2017).

Fábri et al. (2019) reported the extension of the time-independent nuclear-motion code GENIUSH with time-dependent quantum-dynamical features and results are presented there for the coherent inhibition and enhancement of tunneling in $\mathrm{NH}_{3}$ isotopomers. The time-dependent Schrödinger equation was solved in the basis of the rovibrational eigenstates of the isolated molecule and the interaction of the molecule with a classical electromagnetic field was described within the framework of the electric dipole approximation. The rovibrational eigenstates were computed using contracted vibrational basis functions and the vibrational subspace method introduced in Section 3.1. The quantum-dynamical schemes utilized in Fábri et al. (2019) rely heavily on the concept of light-dressed states (Grossmann et al., 1991, Holthaus, 1992) and achieve the coherent inhibition and enhancement of tunneling by nonresonant laser fields. An important feature of the work of Fábri et al. (2019) is that, in contrast to previous vibration-only studies (Marquardt et al., 2003, 2010, Gatti and Marquardt, 2012, Sala et al., 2012 , 2014), all rotational and vibrational degrees of freedom are treated in a numerically exact way and neither the alignment nor the orientation of the molecule are assumed.

\section{Summary and outlook}

Molecules are assemblies of electrons and nuclei. "Effects" due to the rotational and/or the vibrational motions of the nuclei are omnipresent whether one takes them into account explicitly or not. Nevertheless, the Born-Oppenheimer separation of the motion of the electrons and the nuclei is a remarkably good approximation for most of chemistry. As a result, in the majority of quantum-dynamics computations one first solves the electronic motion problem adiabatically decoupled from the motions of the nuclei. This can be done today in an almost black-box-like 
fashion with outstanding accuracy for quite large systems. Then the motion of the nuclei is considered on a potential energy hypersurface (PES) provided by electronic-structure theory.

One of the simplest dynamical descriptions of molecules in motion is given by considering the classical movement of the nuclei on a PES. This classical description is useful in a semiquantitative understanding of many dynamical processes. Nevertheless, the zero-point energy of molecules and the tunneling of nuclei, most importantly that of protons, are not readily incorporated in this model and must be considered as special "effects", showing limitations of this approach. Thus, one needs to move toward a quantum treatment of all the particles.

Spectroscopy, dealing with the results of the interaction between matter and electromagnetic radiation, has been one of the greatest inventions of humankind. Its often extreme accuracy and precision facilitates the understanding of the world around us from the extremely small (atoms and molecules) to the extremely large (astronomical) objects. The remarkable successes of experimental and observational spectroscopy could not have happened without the help from theory, mostly quantum chemistry and molecular physics. Spectroscopic experiments provide a very large amount of data that need to be interpreted and explained and the aim of a large part of nuclear-motion computations is exactly this.

In the fourth age of quantum chemistry the algorithms and the codes developed became sophisticated enough that they allow the interpretation of most high-resolution spectroscopic measurements. Nevertheless, black-box-type techniques, like those of electronic structure theory, still do not exist in nuclear-motion theory though the field is moving toward that direction with considerable pace. Attempts to develop black-box-type quantum dynamics techniques will remain with us for the foreseeable future.

As in all fields under intense development, there are various trends within nuclear-motion theory. These include time-dependent and time-independent descriptions, perturbational and variational algorithms, various forms of rovibrational Hamiltonians, as well as different numerical representations of the Hamiltonians. In this chapter only time-independent variational techniques have been treated in detail. One may wonder which is the best combination of the numerous possible choices or whether a unified protocol would soon emerge.

At this point we have to leave these questions open and say that at present the application in question determines the most appropriate choice. What can be said with confidence is that the extensive use of the different algorithms and protocols indicates that the current quantumchemical adaptation of quantum mechanics to molecular systems is highly successful all the way from the semirigid to the quasistructural regime. As a result, the outcome of quantumchemical simulations based on the technology developed can be used in several applied fields of science and engineering, an example is the improvement of the outcome of atmospheric radiative models.

In the most modern times we are witnessing the merging of time-independent and time-dependent approaches. This is due to the fact that time-dependent nuclear motion computations may follow two basic approaches, a direct or an indirect one. The direct approach implies the numerical solution of the time-dependent Schrödinger equation by an appropriate time-propagation method, not requiring the usually expensive computation of eigenstates. In the indirect approach the time-dependent problem is solved in two consecutive steps. First, the time-independent 
Schrödinger equation is solved via techniques described in this chapter, resulting in rovibrational energy levels and eigenstates. The results of this step can be compared with experimental transitions and transition moments. In the second step the time-dependent wavefunction is represented in the basis of eigenstates and the resulting set of coupled first-order differential equations is solved, yielding the time-dependent wavefunction and other time-dependent molecular quantities. This step can be executed either for the isolated molecule or for the system interacting with an electromagnetic field. In the second step one can design suitable approximations, such as the quasiresonant or the Floquet-Lyapunov approximation for coherent excitation, which facilitate long-time propagation, not easily amenable to direct approaches.

Without discounting the considerable achievements of nuclear-motion theory, it must also be stressed that there are several outstanding challenges which should be met in the near future and require further developments of the existing methodologies. The so-called Carrington bands close to the first dissociation limit of the molecular ion $\mathrm{H}_{3}^{+}$still remain unassigned. In particular, efficient and black-box-type computation of resonance states requires further concerted method developments. Treatment of highly-excited states of semirigid molecules, of almost all rovibronic states of molecules "with no structure" (called quasistructural molecules, like $\mathrm{CH}_{5}^{+}$), as well as of weakly-bound molecular systems, like those held together by dispersive interactions, is far from being solved, especially not in full dimension. Computation of the hyperfine structure of measured spectra as well as the efficient joint treatment of several PESs await further extensive studies. Extending the applicability of nearly exact nuclear-motion treatments to much larger, flexible molecules requires further significant efforts. Quantum-chemical computation of rate constants and advancing quantum control are two areas where a lot of developments are expected in the near future. In summary, it is clear that in the fourth age of quantum chemistry method development and the application of the new techniques to outstanding problems of chemistry continue and continue with a hopefully increased speed. 


\section{References}

K. Aarset, A. G. Császár, E. L. Sibert III, W. D. Allen, H. F. Schaefer III, W. Klopper, and J. Noga. Anharmonic force field, vibrational energy levels, and barrier to inversion of $\mathrm{SiH}_{3}^{-}$. J. Chem. Phys., 112:4053-4063, 2000.

A. R. Al Derzi, T. Furtenbacher, J. Tennyson, S. N. Yurchenko, and A. G. Császár. Marvel analysis of the measured high-resolution spectra of ${ }^{14} \mathrm{NH}_{3}$. J. Quant. Spectrosc. Rad. Transfer, 161:117-130, 2015.

S. Albert, P. Lerch, R. Prentner, and M. Quack. Tunneling and tunneling switching dynamics in phenol and its isotopomers from high-resolution FTIR spectroscopy with synchrotron radiation. Angew. Chem. Int. Ed., 52:346-349, 2013.

S. Albert, Z. Chen, C. Fábri, P. Lerch, R. Prentner, and M. Quack. A combined Gigahertz and Terahertz (FTIR) spectroscopic investigation of meta-D-phenol: Observation of tunneling switching. Mol. Phys., 114:2751-2768, 2016.

W. D. Allen, Y. Yamaguchi, A. G. Császár, D. A. Clabo Jr., R. B. Remington, and H. F. Schaefer III. A systematic study of molecular vibrational anharmonicity and vibration-rotation interaction by self-consistent-field higher derivative methods. Linear polyatomic molecules. Chem. Phys., 145:427-466, 1990.

E. A. G. Armour, J.-M. Richard, and K. Varga. Stability of few-charge systems in quantum mechanics. Phys. Rep., 413:1-90, 2005.

O. Asvany, P. Padma Kumar, B. Redlich, I. Hegemann, S. Schlemmer, and D. Marx. Understanding the infrared spectrum of bare $\mathrm{CH}_{5}^{+}$. Science, 309:1219-1222, 2005.

O. Asvany, K. M. T. Yamada, S. Brünken, A. Potapov, S. Schlemmer, and D. Marx. Experimental ground-state combination differences of $\mathrm{CH}_{5}^{+}$. Science, 347:1346-1349, 2015.

R. J. Barber, J. Tennyson, G. J. Harris, and R. N. Tolchenov. A high-accuracy computed water line list. Mon. Not. R. Astron. Soc., 368:1087-1094, 2006.

P. Barletta, S. V. Shirin, N. F. Zobov, O. L. Polyansky, J. Tennyson, E. F. Valeev, and A. G. Császár. The CVRQD ab initio ground-state adiabatic potential energy surfaces for the water molecule. J. Chem. Phys., 125:204307, 2006.

V. Barone, M. Biczysko, and J. Bloino. Fully anharmonic IR and Raman spectra of medium-size molecular systems: accuracy and interpretation. Phys. Chem. Chem. Phys., 16:1759-1787, 2014.

Z. Bačić and J. C. Light. Theoretical methods for rovibrational states of floppy molecules. Annu. Rev. Phys. Chem., 40:469-498, 1989.

D. Baye and P. H. Heenen. Generalised meshes for quantum mechanical problems. J. Phys. A, 19:2041, 1986. 
M. H. Beck, A. Jäckle, G. A. Worth, and H.-D. Meyer. The multiconfiguration time-dependent Hartree method: A highly efficient algorithm for propagating wavepackets. Phys. Rep., 324: $1-105,2000$.

R. P. Bell. The Tunnel Effect in Chemistry. Chapman and Hall, New York, 1980.

R. Blackmore and B. Shizgal. Discrete-ordinate method of solution of Fokker-Planck equations with nonlinear coefficients. Phys. Rev. A, 31:1855-1868, 1985.

M. Blanco and E. J. Heller. Angular momentum projection operators and molecular bound states. J. Chem. Phys., 78:2504-2517, 1983.

M. Bogey, C. Demuynck, J. L. Destombes, and A. D. Walters. Laboratory rotational spectrum of $\mathrm{SiC}_{2}$ in the $v_{3}$ vibrationally excited state. Astron. Astrophys., 247:L13-L16, 1991.

K. F. Bonhoeffer and P. Harteck. Über Para- und Orthowasserstoff. Z. Physik. Chem., 4B:113, 1929.

J. Bordé, C. J. Bordé, C. Salomon, A. Van Lerberghe, M. Ouhayoun, and C. D. Cantrell. Breakdown of the point-group symmetry of vibration-rotation states and optical observation of ground-state octahedral splittings of ${ }^{32} \mathrm{SF}_{6}$ using saturation spectroscopy. Phys. Rev. Lett., 45:14-17, 1980.

M. Born and W. Heisenberg. Zur Quantentheorie der Molekeln. Ann. Phys., 379:1, 1924.

M. Born and J. R. Oppenheimer. Zur Quantentheorie der Molekeln. Ann. Phys., 389:457, 1927.

J. M. Bowman, S. Carter, and X. Huang. MULTIMODE: A code to calculate rovibrational energies of polyatomic molecules. Int. Rev. Phys. Chem., 22:533-549, 2003.

J. M. Bowman, T. Carrington Jr., and H.-D. Meyer. Variational quantum approaches for computing vibrational energies of polyatomic molecules. Mol. Phys., 106:2145-2182, 2008.

B. Braams and J. Bowman. Permutationally invariant potential energy surfaces in high dimensionality. Int. Rev. Phys. Chem., 28:577-606, 2009.

S. Brackertz, S. Schlemmer, and O. Asvany. Searching for new symmetry species of $\mathrm{CH}_{5}^{+}-$ From lines to states without a model. J. Mol. Spectrosc., 342:73-82, 2017.

M. J. Bramley, W. J. Green, and N. C. Handy. Vibration-rotation coordinates and kinetic energy operator for polyatomic molecules. Mol. Phys., 73:1183, 1991.

G. Brocks, A. van der Avoird, B. T. Sutcliffe, and J. Tennyson. Quantum dynamics of non-rigid systems comprising two polyatomic fragments. Mol. Phys., 50:1025-1043, 1983.

J. Broeckhove and L. Lathouwers. Quantum molecular dynamics and angular momentum projection. In C. Cerjan, editor, Numerical Grid Methods and Their Application to Schrödinger's Equation, pages 49-56, Dordrecht, 1993. Springer Netherlands.

P. R. Bunker. A preliminary study of the proton rearrangement energy levels and spectrum of $\mathrm{CH}_{5}^{+}$. J. Mol. Spectrosc., 176:297-304, 1996. 
P. R. Bunker and P. Jensen. Molecular Symmetry and Spectroscopy. NRC Research Press, Ottawa, 1998.

P. R. Bunker and H. C. Longuet-Higgins. The infra-red spectrum of dimethylacetylene and the torsional barrier. Proc. R. Soc. London, Ser. A, 280:340, 1964.

P. R. Bunker, B. Ostojić, and S. Yurchenko. A theoretical study of the millimeterwave spectrum of $\mathrm{CH}_{5}^{+}$. J. Mol. Struct., 695-696:253-261, 2004.

S. Califano. Vibrational States. Wiley, New York, 1970.

G. D. Carney, L. I. Sprandel, and C. W. Kern. Variational approaches to vibration-rotation spectroscopy for polyatomic molecules. Adv. Chem. Phys., 37:305, 1978.

A. Carrington and R. A. Kennedy. Infrared predissociation limit of the $\mathrm{H}_{3}^{+}$ion. J. Chem. Phys., 81:91-112, 1984.

A. Carrington, J. Buttenshaw, and R. A. Kennedy. Observation of the infrared spectrum of $\mathrm{H}_{3}^{+}$ ion at its near dissciation limit. Mol. Phys., 45:753-758, 1982.

T. Carrington. Perspective: Computing (ro-)vibrational spectra of molecules with more than four atoms. J. Chem. Phys., 146:120902, 2017.

T. Carrington Jr. and W. H. Miller. Reaction surface Hamiltonian for the dynamics of reactions in polyatomic systems. J. Chem. Phys., 81:3942-3950, 1984.

S. Carter and N. Handy. A variational method for the determination of the vibrational $(J=$ 0) energy levels of acetylene, using a Hamiltonian in internal coordinates. Comput. Phys. Commun., 51:49-58, 1988.

S. Carter, J. M. Bowman, and L. B. Harding. Ab initio calculations of force fields for $\mathrm{H}_{2} \mathrm{CN}$ and $\mathrm{ClHCN}$ and vibrational energies of $\mathrm{H}_{2} \mathrm{CN}$. Spectrochim. Acta, 53A:1179-1188, 1997a.

S. Carter, S. J. Culik, and J. M. Bowman. Vibrational self-consistent field method for manymode systems: A new approach and application to the vibrations of $\mathrm{CO}$ adsorbed on $\mathrm{Cu}(100)$. J. Chem. Phys., 107:10458-10469, 1997b.

S. Carter, J. M. Bowman, and N. C. Handy. Extensions and tests of MULTIMODE: a code to obtain accurate vibration/rotation energies of many-mode molecules. Theor. Chem. Acc., 100:191-198, 1998.

P. B. Changala. NITROGEN, Numerical and Iterative Techniques for Rovibronic Energies with General Internal Co-ordinates, a program by P. B. Changala, http://www.colorado.edu/nitrogen., 2019.

$\mathrm{X}$. Chapuisat and C. Iung. Vector parametrization of the $N$-body problem in quantum mechanics: Polyspherical coordinates. Phys. Rev. A, 45:6217-6235, 1992.

W. Chen and B. Poirier. Parallel implementation of efficient preconditioned linear solver for grid-based applications in chemical physics. II: QMR linear solver. J. Comput. Phys., 219: 198-209, 2006. 
W. Chen and B. Poirier. Quantum dynamics on massively parallel computers: Efficient numerical implementation for preconditioned linear solvers and eigensolvers. J. Theoret. Comput. Chem., 9:825-846, 2010.

O. Christiansen. Vibrational structure theory: new vibrational wave function methods for calculation of anharmonic vibrational energies and vibrational contributions to molecular properties. Phys. Chem. Chem. Phys., 9:2942-2953, 2007.

D. A. Clabo Jr., W. D. Allen, R. B. Remington, Y. Yamaguchi, and H. F. Schaefer III. A systematic study of molecular vibrational anharmonicity and vibration-rotation interaction by self-consistent-field higher derivative methods. Asymmetric top molecules. Chem. Phys., 123:187, 1988.

E. Clementi, H. Kistenmacher, and H. Popkie. Study of the electronic structure of molecules. XVIII. Interaction between a lithium atom and a cyano group as an example of a polytopic bond. J. Chem. Phys., 58:2460-2466, 1973.

D. T. Colbert and E. L. Sibert III. Variable curvature coordinates for molecular vibrations. J. Chem. Phys., 91:350-363, 1989.

S. M. Colwell and N. C. Handy. The derivation of vibration-rotation kinetic energy operators in internal coordinates. II. Mol. Phys., 92:317-330, 1997.

G. C. Corey and J. W. Tromp. Variational discrete variable representation. J. Chem. Phys., 103: 1812-1820, 1995.

R. D. Cowan and D. C. Griffin. Approximate relativistic corrections to atomic radial wave functions. J. Opt. Soc. Am., 66:1010, 1976.

A. G. Császár and T. Furtenbacher. Spectroscopic networks. J. Mol. Spectrosc., 266:99-103, 2011.

A. G. Császár and T. Furtenbacher. Promoting and inhibiting tunneling via nuclear motions. Phys. Chem. Chem. Phys., 18:1092-1104, 2016.

A. G. Császár and N. C. Handy. The exact quantum mechanical vibrational kinetic energy operator of sequentially-bonded molecules in valence internal coordinates. J. Chem. Phys., 102:3962-3967, 1995a.

A. G. Császár and N. C. Handy. On the form of the exact quantum mechanical vibrational kinetic energy operator for penta-atomic molecules in internal coordinates. Mol. Phys., 86: 959-979, 1995b.

A. G. Császár, W. D. Allen, and H. F. Schaefer III. In pursuit of the ab initio limit for conformational energy prototypes. J. Chem. Phys., 108:9751-9764, 1998a.

A. G. Császár, J. S. Kain, O. L. Polyansky, N. F. Zobov, and J. Tennyson. Relativistic correction to the potential energy surface and vibration-rotation levels of water. Chem. Phys. Lett., 293: 317-323, 1998b. erratum 312:613 (1999). 
A. G. Császár, W. D. Allen, Y. Yamaguchi, and H. F. Schaefer. Ab initio determination of accurate ground electronic state potential energy hypersurfaces for small molecules. In Computational Molecular Spectroscopy, pages 15-68. Wiley, New York, 2000.

A. G. Császár, G. Tarczay, M. L. Leininger, O. L. Polyansky, J. Tennyson, and W. D. Allen. Dream or reality: complete basis set full configuration interaction potential energy hypersurfaces. In Spectroscopy from space, pages 317-339. Kluwer, Dordrecht, 2001.

A. G. Császár, E. Mátyus, T. Szidarovszky, L. Lodi, N. F. Zobov, S. V. Shirin, O. L. Polyansky, and J. Tennyson. First-principles prediction and partial characterization of the vibrational states of water up to dissociation. J. Quant. Spectrosc. Radiat. Transfer, 111:1043-1064, 2010 .

A. G. Császár, C. Fábri, T. Szidarovszky, E. Mátyus, T. Furtenbacher, and G. Czakó. Fourth age of quantum chemistry: Molecules in motion. Phys. Chem. Chem. Phys., 13:1085-1106, 2012.

A. G. Császár, C. Fábri, and J. Sarka. Quasistructural molecules. WIREs Comput. Mol. Sci., page in press, 2019.

J. K. Cullum and R. A. Willoughby. Lanczos Algorithms for Large Symmetric Eigenvalue Computations. Birkhauser, Boston, 1985.

G. Czakó, T. Furtenbacher, A. G. Császár, and V. Szalay. Variational vibrational calculations using high-order anharmonic force fields. Mol. Phys., 102:2411-2423, 2004.

G. Czakó, V. Szalay, and A. G. Császár. Finite basis representations with nondirect product basis functions having structure similar to that of spherical harmonics. J. Chem. Phys., 124: 014110, 2006.

R. Dawes and T. Carrington Jr. A multidimensional discrete variable representation basis obtained by simultaneous diagonalization. J. Chem. Phys., 121:726-736, 2004.

N. De Leon and E. J. Heller. Vector fields, line integrals, and the Hamilton-Jacobi equation: Semiclassical quantization of bound states. Phys. Rev. A, 30:5-18, 1984.

L. M. Delves. Tertiary and general-order collisions. Nucl. Phys., 9:391-399, 1959.

J. Demaison, J. E. Boggs, and A. G. Császár (Eds.). Equilibrium Molecular Structures. CRC Press, Boca Raton, 2011.

A. S. Dickinson and P. R. Certain. Calculation of matrix elements for one-dimensional quantummechanical problems. J. Chem. Phys., 49:4209-4211, 1968.

H. Dosch. Some general aspects of confinement in nanomaterials. Appl. Surf. Sci., 182:192$195,2001$.

A. Y. Dymarsky and K. N. Kudin. Computation of the pseudorotation matrix to satisfy the Eckart axis conditions. J. Chem. Phys., 122:124103, 2005. 
A. L. L. East and P. R. Bunker. A general rotation-contortion Hamiltonian with structure relaxation: Application to the precessing internal rotor model. J. Mol. Spectrosc., 183(1):157-162, 1997.

A. L. L. East, M. Kolbuszewski, and P. R. Bunker. Ab initio calculation of the rotational spectrum of $\mathrm{CH}_{5}^{+}$and $\mathrm{CD}_{5}^{+}$. J. Phys. Chem. A, 101(36):6746-6752, 1997.

J. Echave and D. C. Clary. Potential optimized discrete variable representation. Chem. Phys. Lett., 190:225-230, 1992.

C. Eckart. Some studies concerning rotating axes and polyatomic molecules. Phys. Rev., 47: $552-558,1935$.

T. Ericsson and A. Ruhe. The spectral transformation Lánczos method for the numerical solution of large sparse generalized symmetric eigenvalue problems. Math. Comput., 35:1251$1268,1980$.

C. Fábri and A. G. Császár. Vibrational quantum graphs and their application to the quantum dynamics of $\mathrm{CH}_{5}^{+}$. Phys. Chem. Chem. Phys., 20:16913-16917, 2018.

C. Fábri, G. Czakó, G. Tasi, and A. G. Császár. Adiabatic Jacobi corrections on the vibrational energy levels of $\mathrm{H}_{2}^{+}$isotopologues. J. Chem. Phys., 130:134314, 2009.

C. Fábri, E. Mátyus, and A. G. Császár. Rotating full- and reduced-dimensional quantum chemical models of molecules. J. Chem. Phys., 134:074105, 2011 a.

C. Fábri, E. Mátyus, T. Furtenbacher, L. Nemes, B. Mihály, T. Zoltáni, and A. G. Császár. Variational quantum mechanical and active database approaches to the rotational-vibrational spectroscopy of ketene. J. Chem. Phys., 135:094307, 2011b.

C. Fábri, T. Furtenbacher, and A. G. Császár. A hybrid variational-perturbational nuclear motion algorithm. Mol. Phys., 112:2462-2467, 2014a.

C. Fábri, E. Mátyus, and A. G. Császár. Numerically constructed internal-coordinate Hamiltonian with Eckart embedding and its application for the inversion tunnelling of ammonia. Spectrochim. Acta A, 119:84-89, 2014b.

C. Fábri, J. Sarka, and A. G. Császár. Communication: Rigidity of the molecular ion $\mathrm{H}_{5}^{+}$. J. Chem. Phys., 140:051101, 2014c.

C. Fábri, M. Quack, and A. G. Császár. On the use of nonrigid-molecular symmetry in nuclearmotion computations employing a discrete variable representation: A case study of the bending energy levels of $\mathrm{CH}_{5}^{+}$. J. Chem. Phys., 147:134101, 2017.

C. Fábri, R. Marquardt, A. G. Császár, and M. Quack. Controlling tunneling in ammonia isotopomers. J. Chem. Phys., 150:014102, 2019.

B. Fehrensen, D. Luckhaus, and M. Quack. Inversion tunneling in aniline from high resolution infrared spectroscopy and an adiabatic reaction path Hamiltonian approach. Z. Phys. Chem. (Münich), 209:1, 1999a. 
B. Fehrensen, D. Luckhaus, and M. Quack. Mode selective stereomutation tunnelling in hydrogen peroxide isotopomers. Chem. Phys. Lett., 300:312-320, 1999 b.

B. Fehrensen, D. Luckhaus, M. Quack, M. Wileke, and T. R. Rizzo. Ab initio calculations of mode selective tunneling dynamics in ${ }^{12} \mathrm{CH}_{3} \mathrm{OH}$ and ${ }^{13} \mathrm{CH}_{3} \mathrm{OH}$. J. Chem. Phys., 119:5534, 2003.

P. M. Felker and Z. Bačić. Electric-dipole-coupled $\mathrm{H}_{2} \mathrm{O} \cdot \mathrm{C}_{60}$ dimer: Translation-rotation eigenstates from twelve-dimensional quantum calculations. J. Chem. Phys., 146:084303, 2017.

T. Firmino, R. Marquardt, F. Gatti, and W. Dong. Diffusion rates for hydrogen on $\operatorname{Pd}(111)$ from molecular quantum dynamics calculations. J. Phys. Chem. Lett., 5:4270-4274, 2014.

J. Frenkel. Wave Mechanics, Elementary Theory. Clarendon Press, Oxford, UK, 1932.

K. Fukui. Formulation of the reaction coordinate. J. Phys. Chem., 74:4161-4163, 1970.

K. Fukui. The path of chemical reactions - the IRC approach. Acc. Chem. Res., 14:363-368, 1981.

T. Furtenbacher and A. G. Császár. The role of intensities in determining characteristics of spectroscopic networks. J. Mol. Struct., 1009:123-129, 2012.

T. Furtenbacher, G. Czakó, B. T. Sutcliffe, A. G. Császár, and V. Szalay. The methylene saga continues: Stretching fundamentals and zero-point energy of $\tilde{\mathrm{X}}^{3} B_{1} \mathrm{CH}_{2}$. J. Mol. Struct., 780-781:283, 2006.

T. Furtenbacher, T. Szidarovszky, C. Fábri, and A. G. Császár. MARVEL analysis of the rotational-vibrational states of the molecular ions $\mathrm{H}_{2} \mathrm{D}^{+}$and $\mathrm{D}_{2} \mathrm{H}^{+}$. Phys. Chem. Chem. Phys., 15:10181-10193, 2013a.

T. Furtenbacher, T. Szidarovszky, E. Mátyus, C. Fábri, and A. G. Császár. Analysis of the rotational-vibrational states of the molecular ion $\mathrm{H}_{3}^{+}$. J. Chem. Theory Comp., 9:5471-5478, $2013 b$.

T. Furtenbacher, T. Szidarovszky, J. Hrubý, A. A. Kyuberis, N. F. Zobov, O. L. Polyansky, J. Tennyson, and A. G. Császár. Definitive high-temperature ideal-gas thermochemical functions of the $\mathrm{H}_{2}{ }^{16} \mathrm{O}$ molecule. J. Phys. Chem. Ref. Data, 45:043104, 2016.

F. Gatti and C. Iung. Exact and constrained kinetic energy operators for polyatomic molecules: The polyspherical approach. Phys. Rep., 484:1-69, 2009.

F. Gatti and R. Marquardt. Stereomutation in vibrationally excited $\mathrm{NHD}_{2}$. Comput. Theor. Chem., 990:90-93, 2012.

H. Goldstein, C. P. Poole Jr., and J. L. Safko. Classical Mechanics. Pearson, Harlow, 3rd edn., 2014.

I. Gordon, L. Rothman, C. Hill, R. V. Kochanov, Y. Tan, P. Bernath, M. Birk, V. Boudon, A. Campargue, K. V. Chance, B. J. Drouin, J.-M. Flaud, R. R. Gamache, D. Jacquemart, V. I. Perevalov, A. Perrin, M. A. H. Smith, J. Tennyson, H. Tran, V. G. Tyuterev, G. C. Toon, 
J. T. Hodges, K. P. Shine, A. Barbe, A. G. Császár, M. V. Devi, T. Furtenbacher, J. J. Harrison, A. Jolly, T. Johnson, T. Karman, I. Kleiner, A. Kyuberis, J. Loos, O. M. Lyulin, S. N. Mikhailenko, N. Moazzen-Ahmadi, H. S. P. Müller, O. V. Naumenko, A. V. Nikitin, O. L. Polyansky, M. Rey, M. Rotger, S. Sharpe, E. Starikova, S. A. Tashkun, J. Vander Auwera, G. Wagner, J. Wilzewski, P. Wcislo, S. Yu, and E. Zak. The HITRAN2016 molecular spectroscopic database. J. Quant. Spectrosc. Radiat. Transfer, 203:3-69, 2017.

F. Grossmann, T. Dittrich, P. Jung, and P. Hänggi. Coherent destruction of tunneling. Phys. Rev. Lett., 67:516-519, 1991.

R. Güsten, H. Wiesemeyer, D. Neufeld, K. M. Menten, U. U. Graf, K. Jacobs, B. Klein, O. Ricken, C. Risacher, and J. Stutzki. Astrophysical detection of the helium hydride ion $\mathrm{HeH}^{+}$. Nature, 568:357-359, 2019.

G. Halász and A. Vibók. Using a multi-step potential as an exact solution of the absorbing potential problem on the grid. Chem. Phys. Lett., 323:287-292, 2000.

B. L. Hammond, W. A. Lester Jr., and P. J. Reynolds. Monte Carlo Methods in ab initio Quantum Chemistry. World Scientific, Singapore, 1994.

C. M. Handley and P. L. A. Popelier. Potential energy surfaces fitted by artificial neural networks. J. Phys. Chem. A, 114:3371-3383, 2010.

N. C. Handy. The derivation of vibration-rotation kinetic energy operators, in internal coordinates. Mol. Phys., 61:207-223, 1987.

N. C. Handy, Y. Yamaguchi, and H. F. Schaefer III. The diagonal correction to the BornOppenheimer approximation: Its effect on the singlet-triplet splitting of $\mathrm{CH}_{2}$ and other molecular effects. J. Chem. Phys., 84:4481-4484, 1986.

I. Haritan and N. Moiseyev. On the calculation of resonances by analytic continuation of eigenvalues from the stabilization graph. J. Chem. Phys., 147:014101, 2017.

D. O. Harris, G. G. Engerholm, and W. D. Gwinn. Calculation of matrix elements for onedimensional quantum-mechanical problems and the application to anharmonic oscillators. $J$. Chem. Phys., 43:1515-1517, 1965.

M. A. Harthcock and J. Laane. Calculation of kinetic energy terms for the vibrational Hamiltonian: Application to large-amplitude vibrations using one-, two-, and three-dimensional models. J. Mol. Spectrosc., 91:300-324, 1982.

A. U. Hazi and H. S. Taylor. Stabilization method of calculating resonance energies: Model problem. Phys. Rev. A, 1:1109-1120, 1970.

T. Helgaker, P. Jørgensen, and J. Olsen. Molecular Electronic-Structure Theory. Wiley, Chichester, 2000.

J. R. Henderson and J. Tennyson. DVR1D: Programs for mixed pointwise/basis set calculation of ro-vibrational spectra. Comp. Phys. Commun., 75:365-378, 1993.

E. Herbst. The astrochemistry of $\mathrm{H}_{3}^{+}$. Phil. Trans. R. Soc. Lond. A, 358:2523-2534, 2000. 
G. Herzberg. Molecular spectra and molecular structure, Vols. 1-3. Van Nostrand Reinhold, Melbourne, 1945.

D. Hestenes. New Foundations for Classical Mechanics. Kluwer Academic Publishers, Dordrecht, 2 edition, 1999.

J. O. Hirschfelder and J. S. Dahler. The kinetic energy of relative motion. Proc. Natl. Acad. Sci., 42:363-365, 1956.

M. Holthaus. Pulse-shape-controlled tunneling in a laser field. Phys. Rev. Lett., 69:1596-1599, 1992.

J. T. Hougen, P. R. Bunker, and J. W. C. Johns. The vibration-rotation problem in triatomic molecules allowing for a large-amplitude bending vibration. J. Mol. Spectrosc., 34:136-172, 1970.

A. R. Hoy, I. M. Mills, and G. Strey. Anharmonic force constant calculations. Mol. Phys., 24: 1265-1290, 1972.

X. Huang, A. B. McCoy, J. M. Bowman, L. M. Johnson, C. Savage, F. Dong, and D. J. Nesbitt. Quantum deconstruction of the infrared spectrum of $\mathrm{CH}_{5}^{+}$. Science, 311:60-63, 2006.

X. Huang, D. W. Schwenke, and T. J. Lee. An accurate global potential energy surface, dipole moment surface, and rovibrational frequencies for $\mathrm{NH}_{3}$. J. Chem. Phys., 129:214304, 2008.

F. Hund. Zur Deutung der Molekelspektren. I. Z. Phys., 40:742-764, 1927.

C. Iung, F. Ribeiro, and E. L. Sibert III. Comparison of perturbative and variational treatments of molecular vibrations: Application to the vibrational spectrum of HFCO up to $8000 \mathrm{~cm}^{-1}$. J. Phys. Chem. A, 110:5420-5429, 2006.

S. D. Ivanov, O. Asvany, A. Witt, E. Hugo, G. Mathias, B. Redlich, D. Marx, and S. Schlemmer. Quantum-induced symmetry breaking explains infrared spectra of $\mathrm{CH}_{5}^{+}$isotopologues. Nature Chem., 2:298-302, 2010.

C. G. J. Jacobi. Sur l'élimination des noeuds dans le problème des trois corps. Compt. Rend. Acad. Sci. Paris, 15:236-255, 1843.

D. W. Jepsen and J. O. Hirschfelder. Set of co-ordinate systems which diagonalize the kinetic energy of relative motion. Proc. Natl. Acad. Sci., 45:249-256, 1959.

B. Jiang, J. Li, and H. Guo. Potential energy surfaces from high fidelity fitting of ab initio points: the permutation invariant polynomial - neural network approach. Int. Rev. Phys. Chem., 35: 479-506, 2016.

B. R. Johnson. On hyperspherical coordinates and mapping the internal configurations of a three body system. J. Chem. Phys., 73:5051-5058, 1980.

B. R. Johnson. The quantum dynamics of three particles in hyperspherical coordinates. $J$. Chem. Phys., 79:1916-1925, 1983. 
S. Kanfer and M. Shapiro. Inversion of the $\mathrm{CH}_{3} \mathrm{I}\left(\tilde{\mathrm{X}}^{1} A_{1}\right)$ potential by the discrete position operator method. J. Phys. Chem., 88:3964-3968, 1984.

S. Klaiman and I. Gilary. On resonance: A first glance into the behavior of unstable states. Adv. Quantum Chem., 63:1-31, 2012.

N. E. Klepeis, A. L. L. East, A. G. Császár, W. D. Allen, T. J. Lee, and D. W. Schwenke. The $[\mathrm{FHCl}]^{-}$molecular anion: Structural aspects, global surface, and vibrational eigenspectrum. J. Chem. Phys., 99:3865-3897, 1993.

E. L. Klinting, C. König, and O. Christiansen. Hybrid optimized and localized vibrational coordinates. J. Phys. Chem. A, 119:11007-11021, 2015.

M. Kolbuszewski and P. R. Bunker. Potential barriers, tunneling splittings, and the predicted $J=1 \leftarrow 0$ spectrum of $\mathrm{CH}_{5}^{+}$. J. Chem. Phys., 105:3649-3653, 1996.

H. Kono. Extraction of eigenstates from an optically prepared state by a time-dependent quantum-mechanical method. Toward simulation of "intermediate case" radiationless transitions. Chem. Phys. Lett., 214:137-143, 1993.

D. Kosloff and R. Kosloff. A Fourier method solution for the time dependent Schrödinger equation as a tool in molecular dynamics. J. Comput. Phys., 52:35-53, 1983.

I. N. Kozin, M. M. Law, J. Tennyson, and J. M. Hutson. New vibration-rotation code for tetraatomic molecules exhibiting wide-amplitude motion: WAVR4. Comp. Phys. Comm., 163:117-131, 2004.

I. N. Kozin, M. M. Law, J. Tennyson, and J. M. Hutson. Calculating energy levels of isomerizing tetra-atomic molecules. II. The vibrational states of acetylene and vinylidene. J. Chem. Phys., 122:064309, 2005.

S. V. Krasnoshchekov, E. V. Isayeva, and N. F. Stepanov. Determination of the Eckart moleculefixed frame by use of the apparatus of quaternion algebra. J. Chem. Phys., 140:154104, 2014.

H. W. Kroto. Molecular rotation spectra. Dover, New York, 1992.

K. N. Kudin and A. Y. Dymarsky. Eckart axis conditions and the minimization of the rootmean-square deviation: Two closely related problems. J. Chem. Phys., 122:224105, 2005.

J. Laane, M. A. Harthcock, P. M. Killough, L. E. Bauman, and J. M.Cooke. Vector representation of large-amplitude vibrations for the determination of kinetic energy functions. J. Mol. Spectrosc., 91:286-299, 1982.

C. Lanczos. An iteration method for the solution of the eigenvalue problem of linear differential and integral operators. J. Res. Natl. Bur. Stand., 45:255, 1950.

L. D. Landau and E. M. Lifshitz. Quantum mechanics, 3rd ed. Pergamon, Oxford, 1977.

S. Larimian, S. Erattupuzha, E. Lötstedt, T. Szidarovszky, R. Maurer, S. Roither, M. Schöffler, D. Kartashov, A. Baltuška, K. Yamanouchi, M. Kitzler, and X. Xie. Fragmentation of longlived hydrocarbons after strong field ionization. Phys. Rev. A, 93:053405, 2016. 
D. Lauvergnat. A harmonic adiabatic approximation to calculate highly excited vibrational levels of "floppy molecules". J. Chem. Phys., 114:6592-6604, 2001.

D. Lauvergnat. ElVibRot quantum dynamics code, 2019. URL http: //www. 1cp.u-psud. $\mathrm{fr} /$ Pageperso/lauvergnat/ElVibRot.html.

D. Lauvergnat and A. Nauts. Exact numerical computation of a kinetic energy operator in curvilinear coordinates. J. Chem. Phys., 116:8560-8570, 2002.

E. G. Layton. The Fourier-grid formalism: philosophy and application to scattering problems using R-matrix theory. J. Phys. B, 26:2501-2522, 1993.

C. R. Le Sueur, S. Miller, J. Tennyson, and B. T. Sutcliffe. On the use of variational wavefunctions in calculating vibrational band intensities. Mol. Phys., 76:1147-1156, 1992.

R. Lefebvre. Theory of resonances in molecular systems. EuroPhys. News, 16:4-7, 1985.

G. N. Lewis, M. Randall, K. S. Pitzer, and L. Brewer. Thermodynamics. McGraw-Hill, New York, 1961.

J. C. Light and T. Carrington Jr. Discrete variable representations and their utilization. Adv. Chem. Phys., 114:263-310, 2000.

J. C. Light, I. P. Hamilton, and J. V. Lill. Generalized discrete variable approximation in quantum mechanics. J. Chem. Phys., 82:1400-1409, 1985.

J. V. Lill, G. A. Parker, and J. C. Light. Discrete variable representations and sudden models in quantum scattering theory. Chem. Phys. Lett., 89:483-489, 1982.

R. G. Littlejohn and M. Reinsch. Gauge fields in the separation of rotations and internal motions in the $n$-body problem. Rev. Mod. Phys., 69:213-275, 1997.

R. G. Littlejohn, M. Cargo, K. Mitchell, T. Carrington Jr., and B. Poirier. A general framework for discrete variable representation basis sets. J. Chem. Phys., 116:8691-8703, 2002.

H. C. Longuet-Higgins. The symmetry groups of non-rigid molecules. Mol. Phys., 6:445-460, 1963.

J. D. Louck and H. W. Galbraith. Application of orthogonal and unitary group methods to the N-body problem. Rev. Mod. Phys., 44:540-601, 1972.

J. D. Louck and H. W. Galbraith. Eckart vectors, Eckart frames, and polyatomic molecules. Rev. Mod. Phys., 48:69, 1976.

D. Luckhaus. 6D vibrational quantum dynamics: Generalized coordinate discrete variable representation and (a)diabatic contraction. J. Chem. Phys., 113:1329-1347, 2000.

D. Luckhaus. The vibrational spectrum of HONO: Fully coupled 6D direct dynamics. J. Chem. Phys., 118:8797-8806, 2003.

T. J. Lukka. A simple method for the derivation of exact quantum-mechanical vibration-rotation Hamiltonians in terms of internal coordinates. J. Chem. Phys., 102:3945-3955, 1995. 
G. Ma, R. Chen, and H. Guo. Quantum calculations of highly excited vibrational spectrum of sulfur dioxide. I. Eigenenergies and assignments up to $15000 \mathrm{~cm}^{-1}$. J. Chem. Phys., 110: 8408-8416, 1999.

A. Macías and A. Riera. Constrained variational procedure for the calculation of autoionization lifetimes. Phys. Lett. A, 103:377-380, 1984.

A. Macías and A. Riera. Accurate resonance lifetimes from real stabilization graphs. Chem. Phys. Lett., 164:359-363, 1989.

M. Majumder, S. E. Hegger, R. Dawes, S. Manzhos, X.-G. Wang, T. Carrington Jr., J. Li, and H. Guo. Explicitly-correlated MRCI-F12 potential energy surfaces for methane fit with several permutation invariant schemes and full-dimensional vibrational calculations. Mol. Phys., 113:1823-1833, 2015.

M. Majumder, S. A. Ndengue, and R. Dawes. Automated construction of potential energy surfaces. Mol. Phys., 114:1-18, 2016.

P. Maksyutenko, N. F. Zobov, S. V. Shirin, O. L. Polyansky, J. S. Muenter, T. R. Rizzo, and O. V. Boyarkin. Approaching the full set of energy levels of water. J. Chem. Phys., 126: 241101, 2007.

V. A. Mandelshtam, T. R. Ravuri, and H. S. Taylor. Calculation of the density od resonance states using tha stabilization method. Phys. Rev. Lett., 70:1932-1935, 1993.

V. A. Mandelshtam, H. S. Taylor, V. Ryaboy, and N. Moiseyev. Stabilization theory for computing energies and widths of resonances. Phys. Rev. A, 50:2764-2766, 1994.

S. Manzhos and T. Carrington, Jr. Using an internal coordinate gaussian basis and a spacefixed cartesian coordinate kinetic energy operator to compute a vibrational spectrum with rectangular collocation. J. Chem. Phys., 145:224110, 2016.

S. Manzhos, K. Yamashita, and T. Carrington. Using a neural network based method to solve the vibrational Schrödinger equation for $\mathrm{H}_{2} \mathrm{O}$. Chem. Phys. Lett., 474:217-221, 2009.

S. Manzhos, T. Carrington, L. Laverdure, and N. Mosey. Computing the anharmonic vibrational spectrum of $\mathrm{UF}_{6}$ in 15 dimensions with an optimized basis set and rectangular collocation. $J$. Phys. Chem. A, 119:9557-9567, 2015.

R. Marquardt and M. Quack. Global analytical potential energy surfaces for high-resolution molecular spectroscopy and reaction dynamics. In M. Quack and F. Merkt, editors, Handbook of High-resolution Spectroscopy, volume 1, pages 511-549. John Wiley \& Sons, Ltd, 2011.

R. Marquardt, M. Quack, I. Thanopulos, and D. Luckhaus. Tunneling dynamics of the NH chromophore in $\mathrm{NHD}_{2}$ during and after coherent infrared excitation. J. Chem. Phys., 118: 643-658, 2003.

R. Marquardt, M. Sanrey, F. Gatti, and F. Le Quéré. Full-dimensional quantum dynamics of vibrationally highly excited $\mathrm{NHD}_{2}$. J. Chem. Phys., 133:174302, 2010. 
C. C. Marston and G. G. Bálint-Kürti. The Fourier grid Hamiltonian method for bound state eigenvalues and eigenfunctions. J. Chem. Phys., 91:3571-3576, 1989.

I. Matanović, M. Xu, J. W. Moskowitz, J. Eckert, and Z. Bačić. Methane molecules confined in the small and large cages of structure I clathrate hydrate: Quantum six-dimensional calculations of the coupled translation-rotation eigenstates. J. Chem. Phys., 131:224308, 2009.

I. Matanović, J. L. Belof, B. Space, K. Sillar, J. Sauer, J. Eckert, and Z. Bačić. Hydrogen adsorbed in a metal organic framework-5: Coupled translation-rotation eigenstates from quantum five-dimensional calculations. J. Chem. Phys., 137:014701, 2012.

E. Mátyus. On the calculation of resonances in pre-Born-Oppenheimer molecular structure theory. J. Phys. Chem. A, 117:7195-7206, 2013.

E. Mátyus and M. Reiher. Molecular structure calculations: A unified quantum mechanical description of electrons and nuclei using explicitly correlated Gaussian functions and the global vector representation. J. Chem. Phys., 137:024104, 2012.

E. Mátyus, G. Czakó, B. T. Sutcliffe, and A. G. Császár. Variational vibrational calculations with arbitrary potentials using the Eckart-Watson Hamiltonians and the discrete variable representation. J. Chem. Phys., 127:084102, 2007.

E. Mátyus, G. Czakó, and A. G. Császár. Toward black-box-type full- and reduced-dimensional variational (ro)vibrational computations. J. Chem. Phys., 130:134112, 2009.

E. Mátyus, J. Šimunek, and A. G. Császár. On variational computation of a large number of vibrational energy levels and wave functions for medium-sized molecules. J. Chem. Phys., 131:074106, 2009.

E. Mátyus, C. Fábri, T. Szidarovszky, G. Czakó, W. D. Allen, and A. G. Császár. Assigning quantum labels to variationally computed rotational-vibrational eigenstates of polyatomic molecules. J. Chem. Phys., 133:034113, 2010.

E. Mátyus, J. Hutter, U. Müller-Herold, and M. Reiher. On the emergence of molecular structure. Phys. Rev. A, 83:052512, 2011.

J. E. Mayer and M. G. Mayer. Statistical Mechanics. Wiley, New York, 1940.

R. C. Mayrhofer and E. L. Sibert III. Investigating optimal coordinates for describing vibrational motion. Theor. Chim. Acta, 92:107-122, 1995.

J. L. McAfee and B. Poirier. Quantum dynamics of hydrogen interacting with single-walled carbon nanotubes. J. Chem. Phys., 130:064701, 2009.

J. L. McAfee and B. Poirier. Quantum dynamics of hydrogen interacting with single-walled carbon nanotubes: Multiple H-atom adsorbates. J. Chem. Phys., 134:074308, 2011.

A. B. McCoy, D. C. Burleigh, and E. L. Sibert. Rotation-vibration interactions in highly excited states of $\mathrm{SO}_{2}$ and $\mathrm{H}_{2} \mathrm{CO}$. J. Chem. Phys., 95:7449-7465, 1991.

M. L. McKee. Fluctional molecules. WIREs Comput. Mol. Sci., 1:943-951, 2011. 
D. A. McQuarrie. Statistical Mechanics. University Science Books, Sausalito, 2000.

G. C. Mellau. Complete experimental rovibrational eigenenergies of $\mathrm{HCN}$ up to $6880 \mathrm{~cm}^{-1}$ above the ground state. J. Chem. Phys., 134:234303, 2011a.

G. C. Mellau. Highly excited rovibrational states of HNC. J. Mol. Spectrosc., 269:77-85, $2011 b$.

F. Merkt and M. Quack. Molecular quantum mechanics and molecular spectra, molecular symmetry, and interaction of matter with radiation. In M. Quack and F. Merkt, editors, Handbook of High-resolution Spectroscopy, volume 1, page 1-55. John Wiley \& Sons, Ltd, 2011.

H. D. Meyer, F. Gatti, and G. A. Worth. Multidimensional Quantum Dynamics: MCTDH Theory and Applications. John Wiley \& Sons, 2009.

R. Meyer. Flexible models for intramolecular motion, a versatile treatment and its application to glyoxal. J. Mol. Spectrosc., 76:266-300, 1979.

P. G. Mezey. Potential Energy Hypersurfaces. Elsevier, New York, 1987.

W. H. Miller, N. C. Handy, and J. E. Adams. Reaction path Hamiltonian for polyatomic molecules. J. Chem. Phys., 72:99-112, 1980.

I. M. Mills. Vibration-rotation structure in asymmetric- and symmetric-top molecules. In Molecular Spectroscopy: Modern Research, pages 115-140. Academic, New York, 1972.

I. M. Mills and M. Quack. Comment. Mol. Phys., 100:9-10, 2002.

I. M. Mills and H. W. Thompson. Internal rotation in dimethyl acetylene. Proc. R. Soc. London, Ser. A, 226:306, 1954.

M. Mladenović. Rovibrational Hamiltonians for general polyatomic molecules in spherical polar parametrization. I. Orthogonal representations. J. Chem. Phys., 112:1070-1081, 2000a.

M. Mladenović. Rovibrational Hamiltonians for general polyatomic molecules in spherical polar parametrization. II. Nonorthogonal descriptions of internal molecular geometry. J. Chem. Phys., 112:1082-1095, 2000b.

M. Mladenović. Rovibrational Hamiltonians for general polyatomic molecules in spherical polar parametrization. III. Global vs local axis system and angular coordinates. J. Chem. Phys., 113:10524-10534, 2000c.

M. Mladenović. Discrete variable approaches to tetratomic molecules: Part I: DVR(6) and DVR(3)+DGB methods. Spectrochim. Acta A Mol. Biomol. Spectrosc., 58:795-807, 2002a.

M. Mladenović. Discrete variable approaches to tetratomic molecules: Part II: Application to $\mathrm{H}_{2} \mathrm{O}_{2}$ and $\mathrm{H}_{2}$ CO. Spectrochim. Acta A Mol. Biomol. Spectrosc., 58:809-824, $2002 \mathrm{~b}$.

N. Moiseyev. Non-hermitian quantum mechanics. In Non-Hermitian Quantum Mechanics. Cambridge University Press, 2011. 
N. Moiseyev, S. Friedland, and P. R. Certain. Cusps, $\theta$ trajectories, and the complex virial theorem. J. Chem. Phys., 74:4739-4740, 1981.

J. G. Muga, J. P. Palao, B. Navarro, and I. L. Egusquiza. Complex absorbing potentials. Phys. Rep. Rev. section of Phys. Lett., 395:357-426, 2004.

K. Müller. Reaction paths on multidimensional energy hypersurfaces. Angew. Chem. Int. Ed., 19:1-13, 1980.

J. J. Munro, J. Ramanlal, J. Tennyson, and H. Y. Mussa. Properties of high-lying vibrational states of the $\mathrm{H}_{3}^{+}$molecular ion. Mol. Phys., 104:115-125, 2006.

J. N. Murrell, S. Carter, S. C. Farantos, P. Huxley, and A. J. C. Varandas. Molecular Potential Energy Surfaces. Wiley, New York, 1984.

H. Y. Mussa and J. Tennyson. Calculating quasi-bound rotation-vibrational states of $\mathrm{HOCl}$ using massively parallel computers. Chem. Phys. Lett., 366(5-6):449-457, 2002.

A. Nauts and X. Chapuisat. Momentum, quasi-momentum, and Hamiltonian operators in terms of arbitrary curvilinear coordinates, with special emphasis on molecular Hamiltonians. Mol. Phys., 55:1287-1318, 1985.

V. V. Nefedova, A. I. Boldyrev, and J. Simons. Graphical representation of the symmetries of potential energy surfaces. J. Chem. Phys., 98:8801-8809, 1993.

M. Neff and G. Rauhut. Toward large scale vibrational configuration interaction calculations. J. Chem. Phys., 131:124129, 2009.

D. J. Nesbitt and R. Naaman. On apparent spectroscopic rigidity of floppy molecular systems. J. Chem. Phys., 91:3801-3809, 1989.

H. H. Nielsen. The vibration-rotation energies of molecules. Rev. Mod. Phys., 23:90-136, 1951.

I. M. B. Nielsen, W. D. Allen, A. G. Császár, and H. F. Schaefer. Toward resolution of the silicon dicarbide $\left(\mathrm{SiC}_{2}\right)$ saga: Ab initio excursions in the web of polytopism. J. Chem. Phys., 107:1195-1211, 1997.

L. Nordheim. Zur Theorie der thermischen Emission und der Reflexion von Elektronen an Metallen. Z. Phys., 46:833-855, 1928.

T. Oka. Orders of magnitude and symmetry in molecular spectroscopy. In M. Quack and F. Merkt, editors, Handbook of High-resolution Spectroscopy, volume 1, pages 633-658. John Wiley \& Sons, Ltd, 2011.

T. Oka. Taming $\mathrm{CH}_{5}^{+}$, the "enfant terrible" of chemical structures. Science, 347:1313-1314, 2015.

A. Owens, E. J. Zak, K. L. Chubb, S. N. Yurchenko, J. Tennyson, and A. Yachmenev. Simulating electric field interactions with polar molecules using spectroscopic databases. Sci. Rep., 7: 45068, 2017. 
I. Ozier, P. Yi, A. Khosla, and N. F. Ramsey. Direct observation of ortho-para transitions in methane. Phys. Rev. Lett., 24:642-645, 1970.

K. Pachucki and J. Komasa. Rovibrational levels of HD. Phys. Chem. Chem. Phys., 12:91889196, 2010.

R. T. Pack. Coordinates for an optimum CS approximation in reactive scattering. Chem. Phys. Lett., 108:333-338, 1984.

P. Padma Kumar and D. Marx. Understanding hydrogen scrambling and infrared spectrum of bare $\mathrm{CH}_{5}^{+}$based on ab initio simulations. Phys. Chem. Chem. Phys., 8:573-586, 2006.

D. Papoušek and M. R. Aliev. Molecular Vibrational-Rotational Spectra. Elsevier Scientific Publishing, Amsterdam, 1982.

D. Papp, J. Sarka, T. Szidarovszky, A. G. Császár, E. Mátyus, M. Hochlaf, and T. Stoecklin. Complex rovibrational dynamics of the Ar $\cdot \mathrm{NO}^{+}$complex. Phys. Chem. Chem. Phys., 19: 8152-8160, 2017a.

D. Papp, T. Szidarovszky, and A. G. Császár. A general variational approach for computing rovibrational resonances of polyatomic molecules. Application to the weakly bound $\mathrm{H}_{2} \mathrm{He}^{+}$ and $\mathrm{H}_{2} \cdot \mathrm{CO}$ systems. J. Chem. Phys., 147:094106, 2017 b.

D. Papp, A. G. Császár, K. Yamanouchi, and T. Szidarovszky. Rovibrational resonances in $\mathrm{H}_{2} \mathrm{He}^{+}$. J. Chem. Theory Comput., 14:1523-1533, 2018.

H. Partridge and D. W. Schwenke. The determination of an accurate isotope dependent potential energy surface for water from extensive ab initio calculations and experimental data. J. Chem. Phys., 106:4618-4639, 1997.

M. Pavanello, W. C. Tung, and L. Adamowicz. Determination of deuteron quadrupole moment from calculations of the electric field gradient in $\mathrm{D}_{2}$ and HD. Phys. Rev. A, 81:042526, 2010.

M. Pavanello, L. Adamowicz, A. Alijah, N. F. Zobov, I. I. Mizus, O. L. Polyansky, J. Tennyson, T. Szidarovszky, and A. G. Császár. Calibration-quality adiabatic potential energy surfaces for $\mathrm{H}_{3}^{+}$and its isotopologues. J. Chem. Phys., 136:184303, $2012 \mathrm{a}$.

M. Pavanello, L. Adamowicz, A. Alijah, N. F. Zobov, I. I. Mizus, O. L. Polyansky, J. Tennyson, T. Szidarovszky, A. G. Császár, M. Berg, A. Petrignani, and A. Wolf. Precision measurements and computations of transition energies in rotationally cold triatomic hydrogen ions up to the mid-visible spectral range. Phys. Rev. Lett., 108:023002, 2012b.

A. I. Pavlyuchko, S. N. Yurchenko, and J. Tennyson. A hybrid variational-perturbational calculation of the ro-vibrational spectrum of nitric acid. J. Chem. Phys., 142:094309, 2015.

J. Pesonen. Constrained molecular vibration-rotation Hamiltonians: Contravariant metric tensor. J. Chem. Phys., 139:144310, 2013.

J. Pesonen. Eckart frame vibration-rotation Hamiltonians: Contravariant metric tensor. J. Chem. Phys., 140:074101, 2014. 
J. Pesonen and L. Halonen. Recent advances in the theory of vibration-rotation Hamiltonians. Adv. Chem. Phys., 125:269-349, 2003.

C. Petty and B. Poirier. Using ScallT for performing accurate rovibrational spectroscopy calculations for triatomic molecules: A practical guide. Appl. Math., 5:2756-2763, 2014.

C. Petty, R. F. K. Spada, F. B. C. Machado, and B. Poirier. Accurate rovibrational energies of ozone isotopologues up to $J=10$ utilizing artificial neural networks. J. Chem. Phys., 149: 024307, 2018.

J. P. Pique, F. Hartmann, R. Bacis, S. Churassy, and J. B. Koffend. Hyperfine-induced ungeradegerade symmetry breaking in a homonuclear diatomic molecule near a dissociation limit: ${ }^{127} I_{2}$ at the ${ }^{2} P_{\frac{3}{2}}-{ }^{2} P_{\frac{1}{2}}$ limit. Phys. Rev. Lett., 52:267-270, 1984.

B. Podolsky. Quantum-mechanically correct form of Hamiltonian function for conservative systems. Phys. Rev., 32:812-816, 1928.

B. Poirier. Exploiting both $C_{3 \mathrm{v}}$ symmetry and sparsity in vibrational calculations for methanelike molecules. J. Chem. Phys., 119:90-93, 2003a.

B. Poirier. Using wavelets to extend quantum dynamics calculations to ten or more degrees of freedom. J. Theor. Comp. Chem., 2:65-72, 2003 b.

B. Poirier. Communication: The $\mathrm{H}_{2} \cdot \mathrm{C}_{60}$ inelastic neutron scattering selection rule: Expanded and explained. J. Chem. Phys., 143:101104, 2015.

B. Poirier and T. Carrington Jr. Semiclassically optimized complex absorbing potentials of polynomial form I. Pure imaginary case. J. Chem. Phys., 118:17-28, 2003 a.

B. Poirier and T. Carrington Jr. Semiclassically optimized complex absorbing potentials of polynomial form. II. Complex case. J. Chem. Phys., 119:77-89, 2003 b.

O. L. Polyansky, A. G. Császár, S. V. Shirin, N. F. Zobov, P. Barletta, J. Tennyson, D. W. Schwenke, and P. J. Knowles. High-accuracy ab initio rotation-vibration transitions for water. Science, 299:539-542, 2003.

O. L. Polyansky, A. Alijah, N. F. Zobov, I. I. Mizus, R. I. Ovsyannikov, J. Tennyson, L. Lodi, T. Szidarovszky, and A. G. Császár. Spectroscopy of $\mathrm{H}_{3}^{+}$based on a new high-accuracy global potential energy surface. Phil. Trans. R. Soc. A, 370:5014-5027, 2012.

O. L. Polyansky, A. A. Kyuberis, N. F. Zobov, J. Tennyson, S. N. Yurchenko, and L. Lodi. ExoMol molecular line lists XXX: a complete high-accuracy line list for water. Mon. Not. Royal Astron. Soc., 480:2597-2608, 2018.

P. Pulay, G. Fogarasi, F. Pang, and J. E. Boggs. Systematic ab initio gradient calculation of molecular geometries, force constants, and dipole moment derivatives. J. Am. Chem. Soc., 101:2550-2560, 1979.

P. Pyykkö, K. G. Dyall, A. G. Császár, G. Tarczay, O. L. Polyansky, and J. Tennyson. Lamb shift effects in rotation-vibration spectra of water. Phys. Rev. A, 63:024502, 2001. 
M. Quack. Detailed symmetry selection rules for reactive collisions. Mol. Phys., 34:477-504, 1977.

M. Quack. On the measurement of the parity violating energy difference between enantiomers. Chem. Phys. Lett., 132:147-153, 1986.

M. Quack. Fundamental symmetries and symmetry violations from high-resolution spectroscopy. In M. Quack and F. Merkt, editors, Handbook of High-resolution Spectroscopy, volume 1, pages 659-722. John Wiley \& Sons, Ltd, 2011.

C. R. Quade. Internal coordinate formulation for the vibration-rotation energies of polyatomic molecules. J. Chem. Phys., 64:2783-2795, 1976.

H. M. Quiney, P. Barletta, G. Tarczay, A. G. Császár, O. L. Polyansky, and J. Tennyson. Twoelectron relativistic corrections to the potential energy surface and vibration-rotation levels of water. Chem. Phys. Lett., 344:413-420, 2001.

R. Radau. Sur une transformation des équations différentielles de la dynamique. Ann. Sci. Ecol. Norm. S., 5:311, 1868.

G. Rauhut. Efficient calculation of potential energy surfaces for the generation of vibrational wave functions. J. Chem. Phys., 121:9313-9322, 2004.

G. Rauhut. Configuration selection as a route towards efficient vibrational configuration interaction calculations. J. Chem. Phys., 127:184109, 2007.

A. Riera. Some properties and pitfalls of the stabilization method. A unified approach. J. Phys. Chem., 97:1558-1565, 1993.

U. V. Riss and H. D. Meyer. Calculation of resonance energies and widths using the complex absorbing potential method. J. Phys. B, 26:4503-4536, 1993.

Y. Saad. Iterative Methods for Sparse Linear Systems. Society for Industrial and Applied Mathematics, Philadelphia, PA, 2003.

K. Sadri, D. Lauvergnat, F. Gatti, and H.-D. Meyer. Rovibrational spectroscopy using a kinetic energy operator in Eckart frame and the multi-configuration time-dependent hartree (MCTDH) approach. J. Chem. Phys., 141:114101, 2014.

M. Sala, S. Guérin, F. Gatti, R. Marquardt, and H.-D. Meyer. Laser-induced enhancement of tunneling in $\mathrm{NHD}_{2}$. J. Chem. Phys., 136:194308, 2012.

M. Sala, F. Gatti, and S. Guérin. Coherent destruction of tunneling in a six-dimensional model of $\mathrm{NHD}_{2}$ : A computational study using the multi-configuration time-dependent Hartree method. J. Chem. Phys., 141:164326, 2014.

J. Sarka and A. G. Császár. Interpretation of the vibrational energy level structure of the astructural molecular ion $\mathrm{H}_{5}^{+}$and all of its deuterated isotopomers. J. Chem. Phys., 144:154309, 2016. 
J. Sarka, C. Fábri, T. Szidarovszky, A. G. Császár, Z. Lin, and A. B. McCoy. Modelling rotations, vibrations, and rovibrational couplings in astructural molecules - A case study based on the $\mathrm{H}_{5}^{+}$molecular ion. Mol. Phys., 113:1873-1883, 2015.

J. Sarka, A. G. Császár, S. C. Althorpe, D. J. Wales, and E. Mátyus. Rovibrational transitions of the methane-water dimer from intermolecular quantum dynamical computations. Phys. Chem. Chem. Phys., 18:22816-22826, 2016.

J. Sarka, A. G. Császár, and E. Mátyus. Rovibrational quantum dynamical computations for deuterated isotopologues of the methane-water dimer. Phys. Chem. Chem. Phys., 19:1533515345, 2017.

R. Schinke. Photodissociation Dynamics. Cambridge University Press, Cambridge, 1993.

H. Schmiedt, S. Schlemmer, and P. Jensen. Symmetry of extremely floppy molecules: Molecular states beyond rotation-vibration separation. J. Chem. Phys., 143:154302, 2015.

H. Schmiedt, P. Jensen, and S. Schlemmer. Collective molecular superrotation: A model for extremely flexible molecules applied to protonated methane. Phys. Rev. Lett., 117:223002, 2016.

H. Schmiedt, P. Jensen, and S. Schlemmer. Rotation-vibration motion of extremely flexible molecules - The molecular superrotor. Chem. Phys. Lett., 672:34-46, 2017a.

H. Schmiedt, P. Jensen, and S. Schlemmer. The role of angular momentum in the superrotor theory for rovibrational motion of extremely flexible molecules. J. Mol. Spectrosc., 342: 132-137, 2017b.

M. Schnell. Group theory for high-resolution spectroscopy of nonrigid molecules. In M. Quack and F. Merkt, editors, Handbook of High-resolution Spectroscopy, volume 1, pages 607-632. John Wiley \& Sons, Ltd, 2011.

P. R. Schreiner, S.-J. Kim, H. F. Schaefer III, and P. von Ragué Schleyer. $\mathrm{CH}_{5}^{+}$: The neverending story or the final word? J. Chem. Phys., 99:3716-3720, 1993.

P. R. Schreiner, H. P. Reisenauer, F. C. Pickard, A. C. Simmonett, W. D. Allen, E. Mátyus, and A. G. Császár. Capture of hydroxymethylene and its fast disappearance through tunnelling. Nature, 453:906-909, 2008.

K. Schuh, P. Rosenow, M. Kolesik, E. M. Wright, S. W. Koch, and J. V. Moloney. Nonlinear rovibrational polarization response of water vapor to ultrashort long-wave infrared pulses. Phys. Rev. A, 96:043818, 2017.

B. F. Schutz. Geometrical Methods of Mathematical Physics. Cambridge University Press, 1980.

D. W. Schwenke. On the computation of ro-vibrational energy levels of triatomic molecules. Comput. Phys. Comm., 70:1-14, 1992.

D. W. Schwenke. Variational calculations of rovibrational energy levels and transition intensities for tetratomic molecules. J. Phys. Chem., 100:2867-2884, 1996. 
P. Seidler, M. B. Hansen, W. Gyorffy, D. Toffoli, and O. Christiansen. Vibrational absorption spectra calculated from vibrational configuration interaction response theory using the lanczos method. J. Chem. Phys., 132:164105, 2010.

E. J. Shipsey. Rotationless molecular vibrations. J. Chem. Phys., 89:5473-5485, 1998.

S. V. Shirin, N. F. Zobov, O. L. Polyansky, and J. Tennyson. High accuracy potential energy surfaces for the $\mathrm{H}_{2}{ }^{16} \mathrm{O}, \mathrm{H}_{2}{ }^{17} \mathrm{O}$ and $\mathrm{H}_{2}{ }^{18} \mathrm{O}$ molecules . J. Chem. Phys., 128:224306, 2008.

B. Shizgal and R. Blackmore. A discrete ordinate method of solution of linear boundary value and eigenvalue problems. J. Comp. Phys., 55:313-327, 1984.

M. Sibaev and D. L. Crittenden. Balancing accuracy and efficiency in selecting vibrational configuration interaction basis states using vibrational perturbation theory. J. Chem. Phys., 145:064106, 2016.

E. L. Sibert III. Theoretical studies of vibrationally excited polyatomic molecules using canonical Van Vleck perturbation theory. J. Chem. Phys., 88:4378-4390, 1988.

I. Simkó, T. Furtenbacher, N. Dénes, T. Szidarovszky, J. Hrubý, N. F. Zobov, O. L. Polyansky, J. Tennyson, and A. G. Császár. Recommended ideal-gas thermochemical functions for heavy water and its substituent isotopologues. J. Phys. Chem. Ref. Data, 46:023104, 2017.

I. Simkó, T. Szidarovszky, and A. G. Császár. Toward automated variational computation of rovibrational resonances. A case study of the $\mathrm{H}_{2}$ dimer. J. Chem. Theory Comput., page in press, 2019.

H. D. Simon. The Lanczos algorithm with partial reorthogonalization. Math. Comp., 42:115$142,1984$.

J. Simons. Resonance state lifetimes from stabilization graphs. J. Chem. Phys., 75:2465-2466, 1981.

S. Skokov, J. Bowman, and V. Mandelshtam. Calculation of resonance states of non-rotating HOCl using an accurate ab initio potential. Phys. Chem. Chem. Phys., 1(6):1279-1282, 1999.

F. T. Smith. Participation of vibration in exchange reactions. J. Chem. Phys., 31:1352-1358, 1959.

F. T. Smith. Diabatic and adiabatic representations for atomic collision problems. Phys. Rev., 179:111-123, 1969.

F. T. Smith. Modified heliocentric coordinates for particle dynamics. Phys. Rev. Lett., 45: 1157-1160, 1980.

L. Song and J. Gao. On the construction of diabatic and adiabatic potential energy surfaces based on ab initio valence bond theory. J. Phys. Chem. A, 112:12925-12935, 2008.

D. S. Sorensen. Implicit application of polynomial filters in a K-step Arnoldi method. SIAM J. Matrix Anal. Appl., 13:357, 1992. 
G. O. Sørensen. A new approach to the Hamiltonian of nonrigid molecules. Top. Curr. Chem., 82:99, 1979.

M. Stanke, D. Kedziera, M. Molski, S. Bubin, M. Barysz, and L. Adamowicz. Convergence of experiment and theory on the pure vibrational spectrum of $\mathrm{HeH}^{+}$. Phys. Rev. Lett., 96: $233002,2006$.

K. Stefanski and H. S. Taylor. New approach to understanding quasiperiodicity in nonintegrable Hamiltonian systems. Phys. Rev. A, 31:2810-2820, 1985.

T. Stoecklin, A. Voronin, and J. C. Rayez. Vibrational quenching of $\mathrm{N}_{2}\left(v=1, j_{\text {rot }}=j\right)$ by ${ }^{3} \mathrm{He}$ : Surface and close-coupling calculations at very low energy. Phys. Rev., 66:042703, 2002.

H. L. Strauss and H. M. Pickett. Conformational structure, energy, and inversion rates of cyclohexane and some related oxanes. J. Am. Chem. Soc., 92:7281-7290, 1970.

D. Strobusch and C. Scheurer. Hierarchical expansion of the kinetic energy operator in curvilinear coordinates for the vibrational self-consistent field method. J. Chem. Phys., 135:124102, $2011 \mathrm{a}$.

D. Strobusch and C. Scheurer. The hierarchical expansion of the kinetic energy operator in curvilinear coordinates extended to the vibrational configuration interaction method. J. Chem. Phys., 135:144101, 2011b.

J. Suarez, S. Farantos, S. Stamatiadis, and L. Lathouwers. A method for solving the molecular schrödinger equation in Cartesian coordinates via angular momentum projection operators. Comput. Phys. Commun., 180:2025-2033, 2009.

B. T. Sutcliffe and J. Tennyson. A general treatment of vibration-rotation coordinates for triatomic molecules. Int. J. Quant. Chem., 39:183-196, 1991.

V. Szalay. Derivation of the nonrigid rotation-large-amplitude internal motion Hamiltonian of the general molecule. J. Mol. Spectrosc., 128:24-61, 1988.

V. Szalay. Discrete variable representations of differential operators. J. Chem. Phys., 99:19781984, 1993.

V. Szalay. The generalized discrete variable representation. An optimal design. J. Chem. Phys., 105:6940-6956, 1996.

V. Szalay. Eckart-Sayvetz conditions revisited. J. Chem. Phys., 140:234107, 2014.

V. Szalay. Understanding nuclear motions in molecules: Derivation of Eckart frame rovibrational Hamiltonian operators via a gateway Hamiltonian operator. J. Chem. Phys., 142: 174107, 2015a.

V. Szalay. Aspects of the Eckart frame ro-vibrational kinetic energy operator. J. Chem. Phys., 143:064104, 2015b.

V. Szalay. Eckart ro-vibrational Hamiltonians via the gateway Hamilton operator: Theory and practice. J. Chem. Phys., 146:124107, 2017. 
V. Szalay, G. Czakó, A. Nagy, T. Furtenbacher, and A. G. Császár. On one-dimensional discrete variable representations with general basis functions. J. Chem. Phys., 119:10512-10518, 2003.

V. Szalay, T. Szidarovszky, G. Czakó, and A. G. Császár. A paradox of grid-based representation techniques: Accurate eigenvalues from inaccurate matrix elements. J. Math. Chem., 50:636$651,2012$.

T. Szidarovszky and A. G. Császár. Low-lying quasibound rovibrational states of $\mathrm{H}_{2}{ }^{16} \mathrm{O}$. Mol . Phys., 111:2131-2146, 2013.

T. Szidarovszky and K. Yamanouchi. Full-dimensional simulation of the laser-induced alignment dynamics of $\mathrm{H}_{2} \mathrm{He}^{+}$. Mol. Phys., 115:1916-1926, 2017.

T. Szidarovszky and K. Yamanouchi. Laser-induced alignment and orientation dynamics beyond the rigid-rotor approximation. In Progress In Ultrafast and Intense Laser Science XIV (in press). Springer Nature Switzerland AG, 2018.

T. Szidarovszky, A. G. Császár, and G. Czakó. On the efficiency of treating singularities in triatomic variational vibrational computations. The vibrational states of $\mathrm{H}_{3}^{+}$up to dissociation. Phys. Chem. Chem. Phys., 12:8373-8386, 2010.

T. Szidarovszky, C. Fábri, and A. G. Császár. The role of axis embedding on rigid rotor decomposition (RRD) analysis of variational rovibrational wave functions. J. Chem. Phys., 136: 174112, 2012.

G. Tarczay, A. G. Császár, W. Klopper, and H. M. Quiney. Anatomy of relativistic energy corrections in light molecular systems. Mol. Phys., 99:1769-1794, 2001.

J. Tennyson. Perspective: Accurate ro-vibrational calculations on small molecules. J. Chem. Phys., 145:120901, 2016.

J. Tennyson and B. T. Sutcliffe. The ab initio calculation of the vibrational-rotational spectrum of triatomic systems in the close-coupling approach, with $\mathrm{KCN}$ and $\mathrm{H}_{2} \mathrm{Ne}$ as examples. $J$. Chem. Phys., 77(8):4061-4072, 1982.

J. Tennyson, M. A. Kostin, P. Barletta, G. J. Harris, O. L. Polyansky, J. Ramanlal, and N. F. Zobov. DVR3D: a program suite for the calculation of rotation-vibration spectra of triatomic molecules. Comput. Phys. Commun., 163:85-116, 2004.

J. Tennyson, P. Barletta, J. J. Munro, and B. C. Silva. The role of asymptotic vibrational states in $\mathrm{H}_{3}^{+}$. Phil. Trans. Royal Soc. London A, 364:2903-2916, 2006.

J. Tennyson, P. F. Bernath, L. R. Brown, A. Campargue, M. R. Carleer, A. G. Császár, R. R. Gamache, J. T. Hodges, A. Jenouvrier, O. V. Naumenko, O. L. Polyansky, L. S. Rothman, R. A. Toth, A. C. Vandaele, N. F. Zobov, L. Daumont, A. Z. Fazliev, T. Furtenbacher, I. F. Gordon, S. N. Mikhailenko, and S. V. Shirin. Critical evaluation of the rotational-vibrational spectra of water vapor. Part I. Energy levels and transition wavenumbers for $\mathrm{H}_{2}{ }^{17} \mathrm{O}$ and $\mathrm{H}_{2}{ }^{18}$ O. J. Quant. Spectrosc. Radiat. Transfer, 110:573-596, 2009. 
J. Tennyson, P. F. Bernath, L. R. Brown, A. Campargue, M. R. Carleer, A. G. Császár, R. R. Gamache, J. T. Hodges, A. Jenouvrier, O. V. Naumenko, O. L. Polyansky, L. S. Rothman, R. A. Toth, A. C. Vandaele, N. F. Zobov, A. Z. Fazliev, T. Furtenbacher, I. F. Gordon, S.M. Hu, S. N. Mikhailenko, and B. Voronin. Critical evaluation of the rotational-vibrational spectra of water vapor. Part II. Energy levels and transition wavenumbers for $\mathrm{HD}^{16} \mathrm{O}, \mathrm{HD}^{17} \mathrm{O}$, and $\mathrm{HD}^{18}$ O. J. Quant. Spectrosc. Radiat. Transfer, 110:2160-2184, 2010.

J. Tennyson, P. F. Bernath, L. R. Brown, A. Campargue, A. G. Császár, L. Daumont, R. R. Gamache, J. T. Hodges, O. V. Naumenko, O. L. Polyansky, L. S. Rothman, A. C. Vandaele, N. F. Zobov, A. R. Al Derzi, C. Fábri, A. Z. Fazliev, T. Furtenbacher, I. E. Gordon, L. Lodi, and I. I. Mizus. IUPAC critical evaluation of the rotational-vibrational spectra of water vapor. Part III: Energy levels and transition wavenumbers for $\mathrm{H}_{2}^{16} \mathrm{O}$. J. Quant. Spectrosc. Rad. Transfer, 117:29-58, 2013.

J. Tennyson, P. F. Bernath, L. R. Brown, A. Campargue, A. G. Császár, L. Daumont, R. R. Gamache, J. T. Hodges, O. V. Naumenko, O. L. Polyansky, L. S. Rothmam, A. C. Vandaele, N. F. Zobov, N. Dénes, A. Z. Fazliev, T. Furtenbacher, I. E. Gordon, S.-M. Hu, T. Szidarovszky, and I. A. Vasilenko. IUPAC critical evaluation of the rotational-vibrational spectra of water vapor. Part IV. Energy levels and transition wavenumbers for $\mathrm{D}_{2}{ }^{16} \mathrm{O}, \mathrm{D}_{2}{ }^{17} \mathrm{O}$ and $\mathrm{D}_{2}{ }^{18}$ O. J. Quant. Spectrosc. Radiat. Transfer, 142:93-108, $2014 \mathrm{a}$.

J. Tennyson, P. F. Bernath, L. R. Brown, A. Campargue, A. G. Császár, L. Daumont, R. R. Gamache, J. T. Hodges, O. V. Naumenko, O. L. Polyansky, L. S. Rothman, A. C. Vandaele, and N. F. Zobov. A database of water transitions from experiment and theory (IUPAC technical report). Pure Appl. Chem., 86:71-83, 2014b.

D. P. Tew, N. C. Handy, and S. Carter. Glyoxal studied with multimode, explicit large amplitude motion and anharmonicity. Phys.Chem.Chem.Phys., 3:1958-1964, 2001.

B. Thomsen, K. Yagi, and O. Christiansen. Optimized coordinates in vibrational coupled cluster calculations. J. Chem. Phys., 140:154102, 2014.

J. Tremblay and T. Carrington. Computing resonance energies, widths, and wave functions using a Lanczos method in real arithmetic. J. Chem. Phys., 122:24, 2005.

E. F. Valeev, W. D. Allen, H. F. Schaefer III, and A. G. Császár. The second-order MøllerPlesset limit for the barrier to linearity of water. J. Chem. Phys., 114:2875-2878, 2001.

T. van Mourik, G. J. Harris, O. L. Polyansky, J. Tennyson, A. G. Császár, and P. J. Knowles. Ab initio global potential, dipole, adiabatic and relativistic correction surfaces for the HCN/HNC system. J. Chem. Phys., 115:3706-3718, 2001.

A. Vibók and G. G. Balint-Kurti. Reflection and transmission of waves by a complex potential - a semiclassical Jeffreys-Wentzel-Kramers-Brillouin treatment. J. Chem. Phys., 96:7615$7622,1992$.

J. Šmydke and A. G. Császár. On the use of reduced density matrices for the semi-automatic assignment of vibrational states. Mol. Phys., 2019. doi: 10.1080/00268976.2018.1562124. 
J. Šmydke, C. Fábri, J. Sarka, and A. G. Császár. Rovibrational quantum dynamics of the vinyl radical and its deuterated isotopologues. Phys. Chem. Chem. Phys., 21:3453-3472, 2019.

V. Špirko. Vibrational anharmonicity and the inversion potential function of $\mathrm{NH}_{3} . \quad J . M o l$. Spectrosc., 101:30-47, 1983.

L.-W. Wang and A. Zunger. Solving Schrödinger's equation around a desired energy: Application to silicon quantum dots. J. Chem. Phys., 100:2394-2397, 1994.

X.-G. Wang and T. Carrington. A finite basis representation Lanczos calculation of the bend energy levels of methane. J. Chem. Phys., 118:6946-6956, 2003.

X.-G. Wang and T. Carrington Jr. A symmetry-adapted Lanczos method for calculating energy levels with different symmetries from a single set of iterations. J. Chem. Phys., 114:1473$1477,2001$.

X.-G. Wang and T. Carrington Jr. New ideas for using contracted basis functions with a Lanczos eigensolver for computing vibrational spectra of molecules with four or more atoms. J. Chem. Phys., 117:6923-6934, 2002.

X.-G. Wang and T. Carrington Jr. Using $C_{3 \mathrm{v}}$ symmetry with polyspherical coordinates for methane. J. Chem. Phys., 119:94-100, 2003a.

X.-G. Wang and T. Carrington Jr. A contracted basis-Lanczos calculation of vibrational levels of methane: Solving the Schrödinger equation in nine dimensions. J. Chem. Phys., 119: 101-117, 2003b.

X.-G. Wang and T. Carrington Jr. Contracted basis Lanczos methods for computing numerically exact rovibrational levels of methane. J. Chem. Phys., 121:2937-2954, 2004.

X.-G. Wang and T. Carrington Jr. Improving the calculation of rovibrational spectra of five-atom molecules with three identical atoms by using a $C_{3 v}\left(G_{6}\right)$ symmetry-adapted grid: Applied to $\mathrm{CH}_{3} \mathrm{D}$ and $\mathrm{CHD}_{3}$. J. Chem. Phys., 123:154303, 2005.

X.-G. Wang and T. Carrington Jr. Vibrational energy levels of $\mathrm{CH}_{5}^{+}$. J. Chem. Phys., 129: 234102, 2008.

X.-G. Wang and T. Carrington Jr. Computing rovibrational levels of methane with curvilinear internal vibrational coordinates and an Eckart frame. J. Chem. Phys., 138:104106, 2013.

X.-G. Wang and T. Carrington Jr. Calculated rotation-bending energy levels of $\mathrm{CH}_{5}^{+}$and a comparison with experiment. J. Chem. Phys., 144:204304, 2016.

X.-G. Wang, T. Carrington Jr., R. Dawes, and A. W. Jasper. The vibration-rotation-tunneling spectrum of the polar and T-shaped-N-in isomers of (NNO) $)_{2}$. J. Mol. Spectrosc., 268:53-65, 2011.

J. K. G. Watson. Simplification of the molecular vibration-rotation Hamiltonian. Mol. Phys., 15:479-490, 1968.

J. K. G. Watson. The vibration-rotation Hamiltonian of linear molecules. Mol. Phys., 19:465487, 1970. 
J. K. G. Watson. The molecular vibration-rotation kinetic-energy operator for general internal coordinates. J. Mol. Spectrosc., 228:645-658, 2004.

H. Wei. Eckart frames for planar molecules. J. Chem. Phys., 118:7202-7207, 2003a.

H. Wei. An Eckart-frame kinetic energy operator for tetra-atomic planar molecules. J. Chem. Phys., 118:7208-7214, 2003b.

H. Wei and T. Carrington. The triatomic Eckart-frame kinetic energy operator in bond coordinates. J. Chem. Phys., 107:9493-9501, 1997.

H. Wei and T. Carrington Jr. The discrete variable representation of a triatomic Hamiltonian in bond length-bond angle coordinates. J. Chem. Phys., 97:3029-3037, 1992.

H. Wei and T. Carrington Jr. Explicit expressions for triatomic Eckart frames in Jacobi, Radau, and bond coordinates. J. Chem. Phys., 107:2813-2818, 1997.

H. Wei and T. Carrington Jr. An exact Eckart-embedded kinetic energy operator in Radau coordinates for triatomic molecules. Chem. Phys. Lett., 287:289-300, 1998.

E. T. White, J. Tang, and T. Oka. $\mathrm{CH}_{5}^{+}$: The infrared spectrum observed. Science, 284(5411): 135-137, 1999.

E. P. Wigner. Group Theory and its Application to the Quantum Mechanics of Atomic Spectra. Academic Press, New York, 1959.

E. B. Wilson Jr., J. C. Decius, and P. C. Cross. Molecular Vibrations. McGraw-Hill, New York, 1955.

R. Wodraszka and U. Manthe. $\mathrm{CH}_{5}^{+}$: Symmetry and the entangled rovibrational quantum states of a fluxional molecule. J. Phys. Chem. Lett., 6:4229-4232, 2015.

K. Wu and H. D. Simon. Thick-restart Lanczos method for the symmetric eigenvalue problems. Lawrence Berkeley National Laboratory Report No. 41412, 1998, 1998.

K. Wu, A. Canning, H. D. Simon, and L.-W. Wang. Thick-restart Lanczos method for electronic structure calculations. J. Comp. Phys., 154:156-173, 1999.

R. E. Wyatt. Matrix spectroscopy: Computation of interior eigenstates of large matrices using layered iteration. Phys. Rev. E, 51:3643-3658, 1995.

R. E. Wyatt and J. Z. H. Zhang. Dynamics of Molecules and Chemical Reactions. Marcel Dekker, New York, 1996.

M. Xu, F. Sebastianelli, Z. Bačić, R. Lawler, and N. J. Turro. Quantum dynamics of coupled translational and rotational motions of $\mathrm{H}_{2}$ inside $\mathrm{C}_{60}$. J. Chem. Phys., 128:011101, 2008a.

M. Xu, F. Sebastianelli, Z. Bačić, R. Lawler, and N. J. Turro. $\mathrm{H}_{2}, \mathrm{HD}$, and $\mathrm{D}_{2}$ inside $\mathrm{C}_{60}$ : Coupled translation-rotation eigenstates of the endohedral molecules from quantum fivedimensional calculations. J. Chem. Phys., 129:064313, 2008 b. 
M. Xu, F. Sebastianelli, and Z. Bačić. Coupled translation-rotation eigenstates of $\mathrm{H}_{2}, \mathrm{HD}$, and $\mathrm{D}_{2}$ in the large cage of structure II clathrate hydrate: Comparison with the small cage and rotational Raman spectroscopy. J. Phys. Chem. A, 113:7601-7609, 2009a.

M. Xu, F. Sebastianelli, B. R. Gibbons, Z. Bačić, R. Lawler, and N. J. Turro. Coupled translation-rotation eigenstates of $\mathrm{H}_{2}$ in $\mathrm{C}_{60}$ and $\mathrm{C}_{70}$ on the spectroscopically optimized interaction potential: Effects of cage anisotropy on the energy level structure and assignments. J. Chem. Phys., 130:224306, 2009 b.

M. Xu, S. Ye, A. Powers, R. Lawler, N. J. Turro, and Z. Bačić. Inelastic neutron scattering spectrum of $\mathrm{H}_{2} \cdot \mathrm{C}_{60}$ and its temperature dependence decoded using rigorous quantum calculations and a new selection rule. J. Chem. Phys., 139:064309, 2013.

M. Xu, S. Ye, and Z. Bačić. General selection rule in the inelastic neutron scattering spectroscopy of a diatomic molecule confined inside a near-spherical nanocavity. J. Phys. Chem. Lett., 6:3721-3725, 2015.

A. Yachmenev and S. N. Yurchenko. Automatic differentiation method for numerical construction of the rotational-vibrational Hamiltonian as a power series in the curvilinear internal coordinates using the Eckart frame. J. Chem. Phys., 143:014105, 2015.

K. Yagi, M. Keceli, and S. Hirata. Optimized coordinates for anharmonic vibrational structure theories. J. Chem. Phys., 137:204118, 2012.

H. Yu. Two-layer Lanczos iteration approach to molecular spectroscopic calculation. J. Chem. Phys., 117:8190-8196, 2002.

H. Yu. Full-dimensional quantum calculations of vibrational spectra of six-atom molecules. I. Theory and numerical results. J. Chem. Phys., 120:2270-2284, 2004a.

H. Yu. Converged quantum dynamics calculations of vibrational energies of $\mathrm{CH}_{4}$ and $\mathrm{CH}_{3} \mathrm{D}$ using an ab initio potential. J. Chem. Phys., 121:6334-6340, 2004b.

$\mathrm{H}$. Yu. A rigorous full-dimensional quantum dynamics calculation of the vibrational energies of $\mathrm{H}_{3} \mathrm{O}_{2}^{-}$. J. Chem. Phys., 125:204306, 2006.

H.-G. Yu. A coherent discrete variable representation method for multidimensional systems in physics. J. Chem. Phys., 122:164107, 2005.

H.-G. Yu and G. Nyman. A spectral transform Krylov subspace iteration approach to quantum scattering. Chem. Phys. Lett., 298:27-35, 1998.

H.-G. Yu and G. Nyman. A four dimensional quantum scattering study of the $\mathrm{Cl}+\mathrm{CH}_{4} \rightarrow \mathrm{HCl}$ $+\mathrm{CH}_{3}$ reaction via spectral transform iteration. J. Chem. Phys., 110:7233-7244, 1999.

S. N. Yurchenko, M. Carvajal, P. Jensen, H. Lin, J. Zheng, and W. Thiel. Rotation-vibration motion of pyramidal $\mathrm{XY}_{3}$ molecules described in the Eckart frame: Theory and application to $\mathrm{NH}_{3}$. Mol. Phys., 103:359-378, 2005.

S. N. Yurchenko, W. Thiel, and P. Jensen. Theoretical ROvibrational Energies (TROVE): A robust numerical approach to the calculation of rovibrational energies for polyatomic molecules. J. Mol. Spectrosc., 245:126-140, 2007. 
S. N. Yurchenko, A. Yachmenev, and R. I. Ovsyannikov. Symmetry-adapted ro-vibrational basis functions for variational nuclear motion calculations: TROVE approach. J. Chem. Theory Comput., 13:4368-4381, 2017.

D. Zanuttini, F. Gatti, and R. Marquardt. CO quantum dynamics diffusion on $\mathrm{Cu}(100)$. Chem. Phys., 509:3-12, 2018.

R. N. Zare. Angular Momentum: Understanding Spatial Aspects in Chemistry and Physics. Wiley-Interscience, New York, 1988.

J. Z. H. Zhang. Theory and Application of Quantum Molecular Dynamics. World Scientific, Singapore, 1999.

B. Ziegler and G. Rauhut. Efficient generation of sum-of-products representations of highdimensional potential energy surfaces based on multimode expansions. J. Chem. Phys., 144: $114114,2016$.

N. F. Zobov, O. L. Polyansky, C. R. Le Sueur, and J. Tennyson. Vibration-rotation levels of water beyond the Born-Oppenheimer approximation. Chem. Phys. Lett., 260:381-387, 1996.

N. F. Zobov, S. V. Shiron, O. L. Polyansky, J. Tennyson, P.-F. Coheur, P. F. Bernath, M. Carleer, and R. Colin. Monodromy in the water molecules. Chem. Phys. Lett., 414:193-197, 2005.

N. F. Zobov, S. V. Shirin, L. Lodi, B. C. Silva, J. Tennyson, A. G. Császár, and O. L. Polyansky. First-principles rotation-vibration spectrum of water above dissociation. Chem. Phys. Lett., 507:48-51, 2011. 